\title{
OPTIMIZATION MODELS FOR DISTRIBUTION PLANNING AND OPERATION
}

\author{
by
}

\author{
Kamran Masteri Farahani \\ Master of Applied Science in Engineering, Ryerson University, Canada, 2012 \\ Bachelor of Engineering, Ryerson University, Canada, 2010
}

\author{
A dissertation \\ presented to Ryerson University \\ in partial fulfillment of the \\ requirements for the degree of \\ Doctor of Philosophy \\ in the Program of
}

Electrical and Computer Engineering

Toronto, Ontario, Canada, 2018

(C) Kamran Masteri, 2018 


\section{AUTHOR's DeClaration}

I hereby declare that I am the sole author of this dissertation. This is a true copy of the dissertation, including any required final revisions, as accepted by my examiners.

I authorize Ryerson University to lend this dissertation to other institutions or individuals for the purpose of scholarly research.

I further authorize Ryerson University to reproduce this dissertation by photocopying or by other means, in total or in part, at the request of other institutions or individuals for the purpose of scholarly research.

I understand that my dissertation may be made electronically available to the public. 


\title{
Abstract
}

\section{Optimization Models for Distribution Planning and Operation}

\author{
Kamran Masteri Farahani \\ Doctor of Philosophy \\ Electrical and Computer Engineering \\ Ryerson University, 2018
}

Smart grid technologies, renewables, energy storage devices and electric vehicles are going to characterize the next generation distribution systems. It is important to note that inclusion of electric vehicles and renewables, inherently due to their natural power profile, result in distribution systems having a peaky load profile with lower asset utilization factors. Optimal planning and operation of distribution systems are important aspects and should consider this changing paradigm.

This thesis aims to develop new solutions for optimal planning and operation of distribution systems considering these new technologies and their implications. The thesis specifically aims to use new techniques such as complementarity in conjunction with classical optimization techniques to develop new algorithms for optimal planning and operation of distribution systems. The proposed work includes the following. Two new distribution planning algorithms are proposed that include the installation and optimal sizing of Battery Energy Storage System units in addition to traditional assets, such as feeders and transformers. It incorporates plan and asset lifetimes as a means of establishing the minimum total annualized costs of new and replacement assets, operation and 
maintenance, and customer interruptions. For a fair comparison, all costs reflect the current year and are annualized over a specific study period. Even though the second technique has the same base as the first method, it is a multi-objective algorithm that uses fuzzy optimization technique to handle multiple contradicting objectives that cannot be combined into a single objective as they are in different units. This method has been developed due to the lack of certainty in how to calculate customer interruption cost in literature. It was proven in both methods that Battery Energy Storage System could be a more economical option compared to expensive underground feeders. Then in order to realize Smart Radial Distribution System of the future, a real-time optimal reconfiguration algorithm is proposed, which uses a classical nonlinear optimization technique and guarantees an optimal solution in the least time. The method optimizes the system loss and is based upon a complementarity technique that transforms a set of discontinuous solution spaces into a single continuously differentiable solution space, thus enabling the use of classical nonlinear optimization techniques without resorting to heuristics. The method is tested on 33-bus and 69-bus systems and the results are better or matching the other methods available in literature while it is significantly faster. 


\section{ACKNOWLEDGMENTS}

First and foremost, I would like to express my sincere gratitude to my supervisor, Dr. Bala Venkatesh, for his patience, motivation, and immense knowledge. His guidance helped me in all the time of research and writing of this thesis. I could not have imagined having a better advisor and mentor for my Ph.D. study.

I was also delighted to interact with the Editor of IEEE Transactions on Power Delivery, Dr. Walmir Freitas. His insights to the distribution systems and the economic side of energy storage devices are second to none. Furthermore, he sets an example of a world-class researcher with his rigor and passion for his work.

I am very grateful to Dr. Mohamed Awadallah for his valuable comments and suggestions. I would also like to thank Dr. Peng Yu, Dr. Chandrabhanu Opathella, and all present and past colleagues in the Power \& Energy Analysis Research (PEAR) Laboratory and Center for Urban Energy (CUE) at Ryerson University for their support and encouragement.

I would also like to acknowledge the support that I received from the faculty and staff in the Department of Electrical and Computer Engineering at Ryerson University in Toronto.

Lastly, I would like to thank my family for their patience, endless support, and encouragement. 


\section{DEDICATION}

To my family, Amir, Fahime and Ghazal 


\section{TABLE OF CONTENTS}

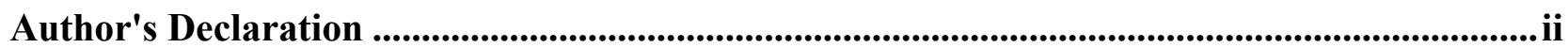

Abstract.............................................................................................................................................ii

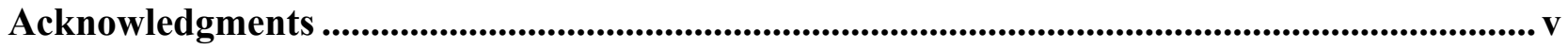

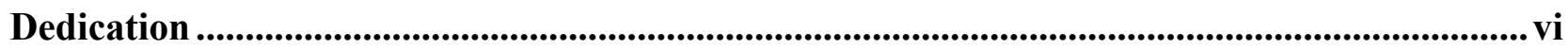

List of Tables ...........................................................................................................................................

List of Figures......................................................................................................................................... xiv

List of Symbols ........................................................................................................................................

CHAPTER 1 INTRODUCTION .................................................................................................................. 1

1.1 Effect of Renewables and Electric Vehicles on Load Profiles .............................................. 5

1.2 Opportunity to Plan with ES as a Cost-Effective Solution .................................................... 8

1.3 Current DS Planning Methods ................................................................................................. 10

1.4 Recent Developments of DS Planning with ES …........................................................... 12

1.5 BESS Model Used in Planning and Evolution of Battery Technology ............................. 17

1.6 Opportunity to Reconfigure and Improve System Performance ........................................ 19

1.7 Current Optimal Reconfiguration Methods .......................................................................... 20 


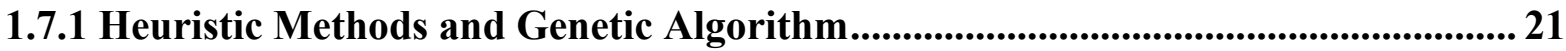

1.7.2 Nonlinear Optimization versus Genetic Algorithms............................................. 22

1.8 Recent Developments of DS Reconfiguration Methods ............................................. 23

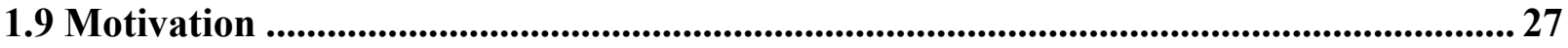

1.9.1 Need for New Methods of DS Planning with ES ....................................................... 27

1.9.2 Need for New Methods of DS Reconfiguration .......................................................... 27

1.10 Objectives of the Thesis...................................................................................................... 28

1.11 Summary of Chapters ..................................................................................................... 29

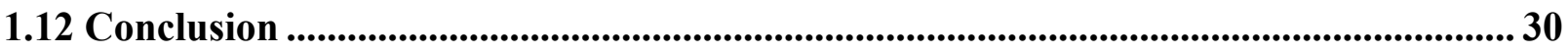

Chapter 2 A Model for Distribution System Asset Planning InCluding Battery

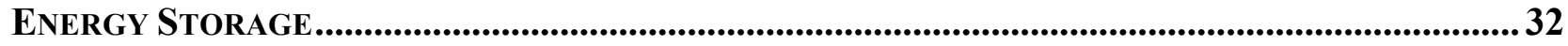

2.1 Problem Formulation of FIM with BESS ............................................................ 33

2.1.1 Objective Function ...................................................................................................... 34

2.1.2 Annualized Capital Costs ........................................................................................... 34

2.1.3 Annualized Risk and Maintenance Cost ..................................................................... 36

2.1.3.1 SAIDI and Customer Interruption Cost Calculation .............................................. 36

2.1.4 Constraints and Boundaries................................................................................ 37

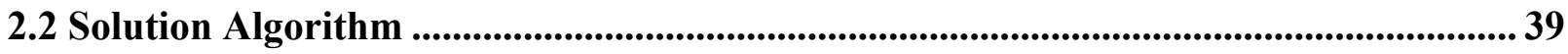

2.3 Test Results and Discussions................................................................................................... 40

2.3.1 Economic Data...................................................................................................................... 40

2.3.2 Simplified Canadian Distribution System Data (3-bus) ............................................. 41

2.3.2.1 Optimal Planning of a Canadian Distribution System ........................................ 47 
2.3.2.2 Sensitivity Analysis for the Canadian Distribution System ...............................53

2.3.3 Optimal Planning for the IEEE 33-bus Distribution System................................ 59

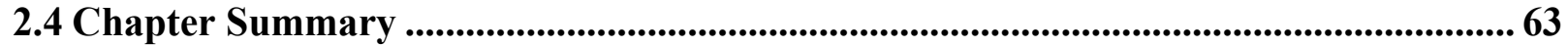

Chapter 3 A Fuzzy Optimization Model for Distribution System Asset Planning

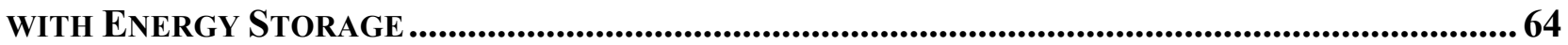

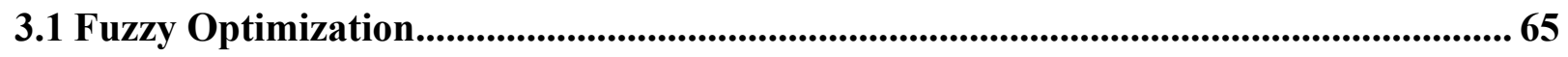

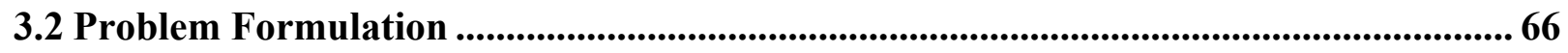

3.2.1 Objective Function ................................................................................................... 67

3.2.1.1 Total Cost Calculation .................................................................................. 67

3.2.1.2 SAIDI Minimization ......................................................................................................... 69

3.2.2 Fuzzy Set of Objective Function ............................................................................ 70

3.2.3 Objective Definition .............................................................................................................. 72

3.2.4 Constraints and Boundaries............................................................................................ 72

3.3 Solution Algorithm .................................................................................................................. 74

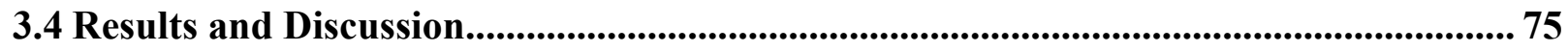

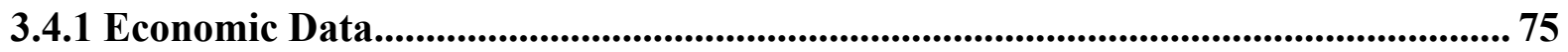

3.4.2 IEEE 33-Bus Distribution System Data .......................................................... 76

3.4.3 Sensitivity Analysis for the 33-Bus Distribution System ............................................. 81

3.4.4 Sensitivity Analysis for the 33-Bus Distribution System ........................................... 84

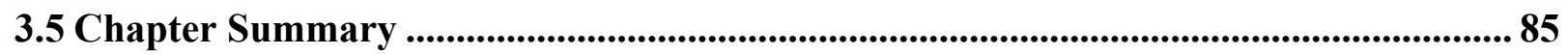

Chapter 4 Real-time SMart Distribution SyStem ReCONFIGURATION USING

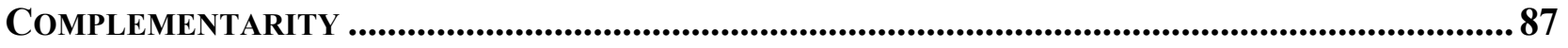


4.2 Complementarity ................................................................................................................................90

4.2.1 Switches and Complementarity ........................................................................................ 92

4.3 Proposed Problem Formulation .......................................................................... 94

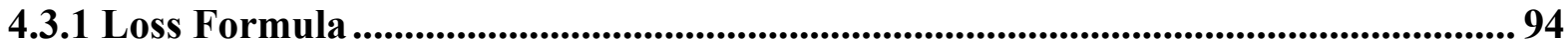

4.3.2 Network Representation ..................................................................................... 94

4.3.3 Network Radial Structure - Complementarity Constraints..................................95

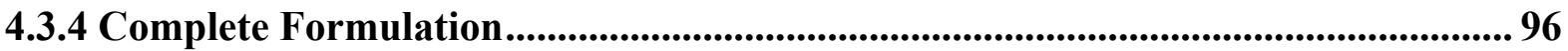

4.4 Results and Discussions .................................................................................................... 97

4.4.1 The 7-bus System .................................................................................................................. 97

4.4.1.1 Result of Proposed Method Using Complementarity .......................................... 98

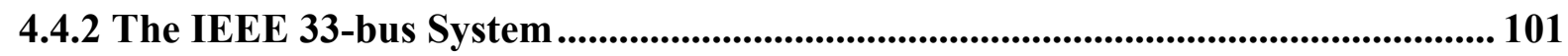

4.4.3 The IEEE 69-bus System ................................................................................... 105

4.5 Chapter Summary

Chapter 5 CONCLUSION AND FUTURE WORK .....................................................................110

5.1 Conclusion and Major Contributions .............................................................................. 110

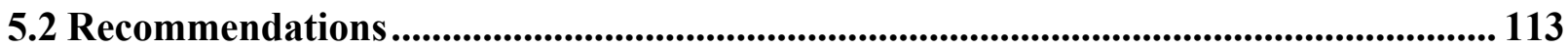

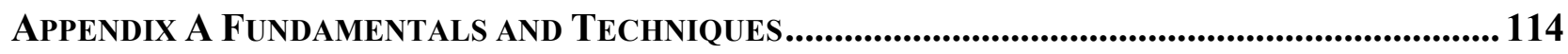

APPENDIX B DATA OF TEST SYSTEMS .......................................................................................... 119

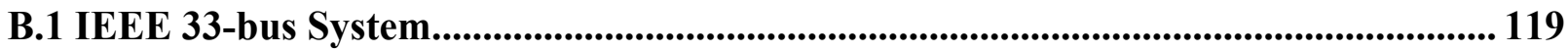

B.2 IEEE 69-bus System.................................................................................................... 122 


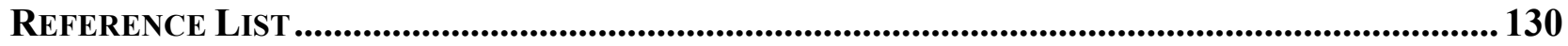




\section{LIST OF TABLES}

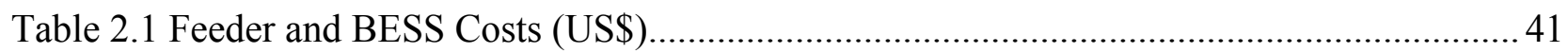

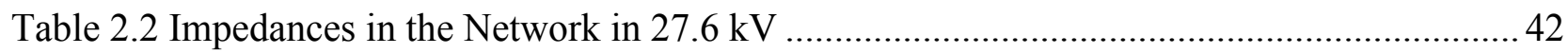

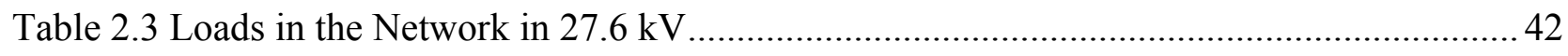

Table 2.4 Base Values for 27.6kV System ..................................................................... 43

Table 2.5 Voltage Solutions from Load Flow .................................................................. 43

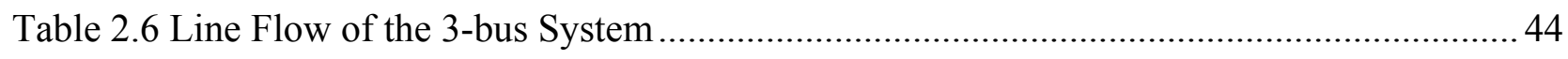

Table 2.7 Power Demand (PD) and PV Generation (PV) Values for Each Time Zone .................45

Table 2.8 Voltage Solutions for Each Time Zone ............................................................... 46

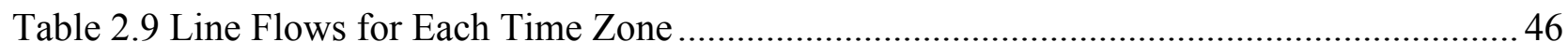

Table 2.10 Total Line Power Flow in the Feeders for the 3-Bus System Cases.......................... 48

Table 2.11 Power Supplied from Feeder Source - Case I.................................................... 49

Table 2.12 Power Supplied from Feeder Source - Case II .................................................... 50

Table 2.13 BESS Power and Energy for Case II ............................................................ 50

Table 2.14 Power Demand (PD) and Power Supplied by the Substation (PG) in MW................55

Table 2.15 Power Demand (PD) and PV Generation (PV) for Each Time Zone in \%.................60

Table 2.16 Total Line Power Flow for the 33-Bus System - Case I .......................................61

Table 2.17 Total Line Power Flow for the 33 Bus System - Case II.......................................62

Table 2.18 Battery Power and Energy for Case II - 33-Bus System.....................................62 62 


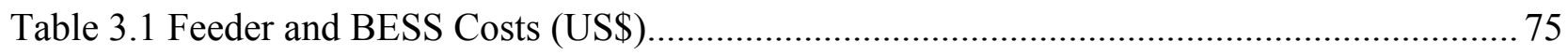

Table 3.2 System Power Demand (PD) Values for Each Time Zone............................................... 77

Table 3.3 Total Line Power Flow for the IEEE 33-Bus System - Case I......................................79

Table 3.4 Total Line Power Flow for the IEEE 33-Bus System - Case II...................................... 80

Table 3.5 Battery Power and Energy for the IEEE 33 Bus System - Case II ................................ 80

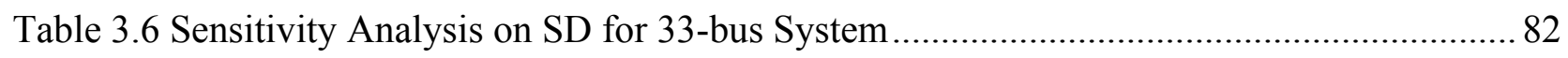

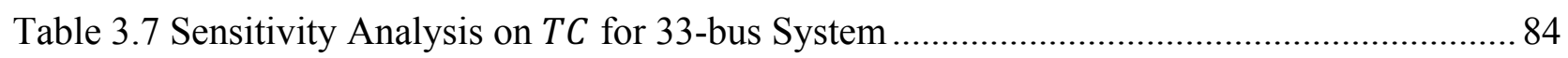

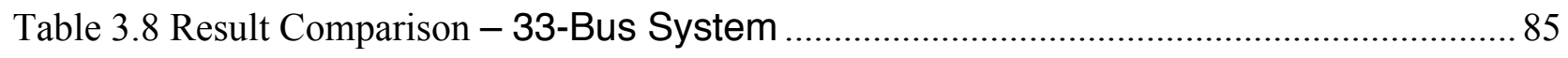

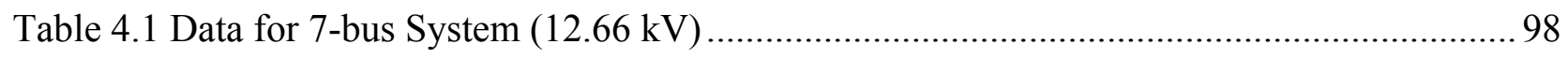

Table 4.2 Comparison of Base Case and Optimal Solution.......................................................... 100

Table 4.3 Comparison of Base Case and Optimal Solution for IEEE 33-bus System.................... 104

Table 4.4 Comparison of Base Case and Optimal Solution for IEEE 69-bus System.................... 106 


\section{LIST OF FIGURES}

Fig. 1.1 Distribution System in the Presence of Renewables, EVs and ESSs .............................. 4

Fig. 1.2 Sample System with Energy Storage System .................................................... 6

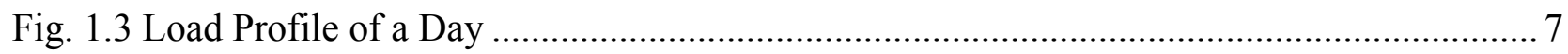

Fig. 1.4 Representation of the Equivalent Annual Costs ..................................................... 12

Fig. 1.5 Battery Power Conversion System Efficiency and Self-Discharge Schematic................. 18

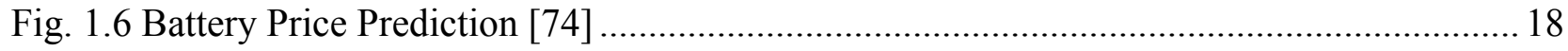

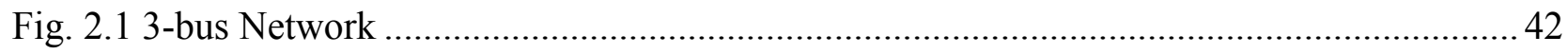

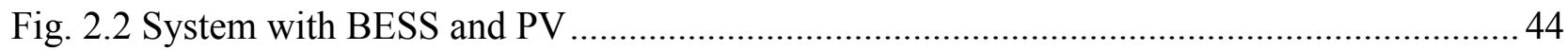

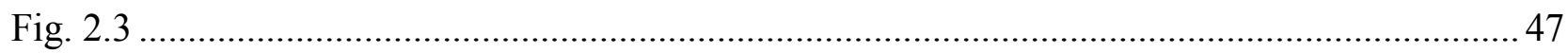

Fig. 2.4 Power Flows in Feeders with No Battery ES - Case I .............................................. 49

Fig. 2.5 Power Flows in Feeders with Battery ES - Case II ................................................5 51

Fig. 2.6 Battery ES Energy and Power Efficiency - Case II.................................................... 52

Fig. 2.7 Loading Factor versus Costs of Feeders and BESS for Constant $\mathrm{P}_{\mathrm{avg}}$ Value and Varied

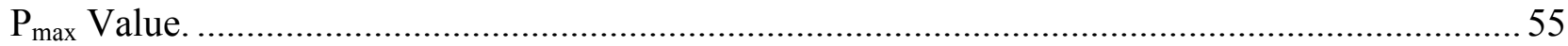

Fig. 2.8 Loading Factor versus Costs of the Feeders and BESS for a Constant $\mathrm{P}_{\max }$ Value and

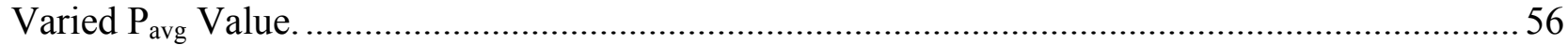

Fig. 2.9 Cost of Utilizing BESS and Feeders versus the BESS Cost Coefficient.........................57

Fig. 2.10 Cost of Utilizing BESS and Feeders versus the Feeder Cost Coefficient ......................59

Fig. 3.1 Fuzzy Satisfaction Functions of Total Cost (TC) .................................................. 70 
Fig. 3.2 Fuzzy Satisfaction Functions of SAIDI (SD) ...................................................... 71

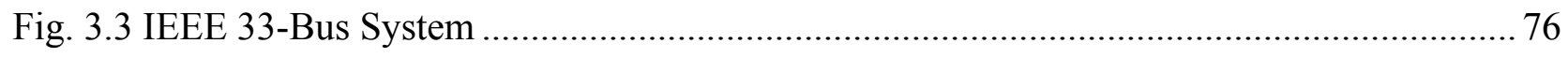

Fig. 3.4 Sensitivity Analysis Based on SD Variation ............................................................ 82

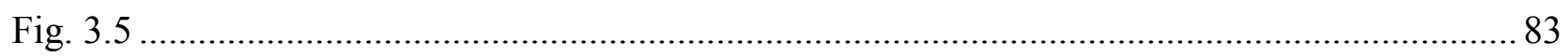

Fig. 4.1 Solution Space for a Sample Problem $(\mathrm{P} 1)$.............................................................. 92

Fig. 4.2 $\mathrm{P}_{\mathrm{L}}$ and $\mathrm{Q}_{\mathrm{L}}$ are Sum of Power Flowing from the Bus into Connected Lines .....................95

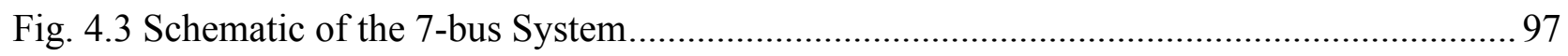

Fig. 4.4 Voltages of Each Individual Bus: 7-bus System ................................................. 99

Fig. 4.5 Comparison of Active-Power Flow of Each Branch Before and After Optimization: 7-bus

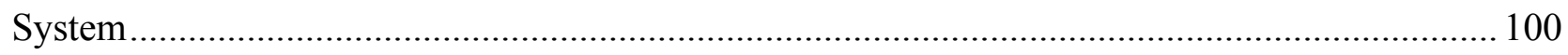

Fig. 4.6 Single Line Diagram of the 33-bus System...................................................... 101

Fig. 4.7 Voltages of Each Individual Bus: IEEE 33-bus System........................................... 102

Fig. 4.8 Comparison of Active-Power Flow of Each Branch Before and After Optimization: IEEE

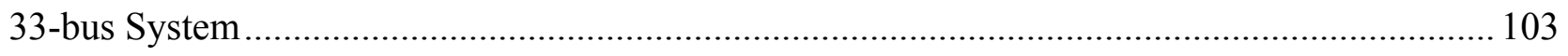

Fig. 4.9 Single Line Diagram of The IEEE 69-bus System.................................................. 105

Fig. 4.10 Voltages of Each Individual Bus: IEEE 69-bus System......................................... 107

Fig. 4.11 Comparison of Active-Power Flow of Each Branch Before and After Optimization: IEEE

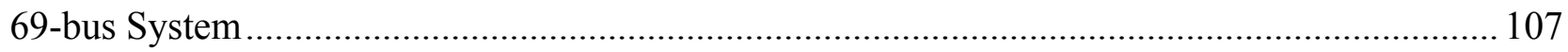




\section{LIST OF SYMBOLS}

\section{FIM Planning Variables}

$\begin{array}{ll}\mathrm{A}, \mathrm{B}, \mathrm{C} & \text { Constant values for calculating SAIDI (Appendix B) } \\ \overline{E B_{l}} & \text { Maximum battery energy, in MWh } \\ \mathrm{BY} & \text { Battery replacement time, in years } \\ f_{S F} & \text { Feeder cost function } \\ f_{B} & \text { Battery cost function } \\ f_{r e p B} & \text { Battery replacement function } \\ f_{C I C} & \text { Customer Interruption cost of the feeder function } \\ f_{m c F} & \text { Maintenance cost of the feeder function } \\ f_{m c B} & \text { Maintenance cost of the battery function } \\ \mathrm{HT}_{\mathrm{t}} & \text { Duration of each time zone, in hours } \\ \mathrm{i}, \mathrm{j} & \text { Bus index } \\ \mathrm{K}_{\mathrm{F}} & \text { Cost for the feeder, in } \$ / \mathrm{MVA} \\ \mathrm{K}_{\mathrm{BP}} & \text { Cost for battery power, in } \$ / \mathrm{MW} \\ \mathrm{K}_{\mathrm{BE}} & \text { Cost fortery energy, in } \$ / \mathrm{MWh} \\ \mathrm{Kr}_{0} & \text { Customer interruption cost, in } \$ / \mathrm{MWh}\end{array}$




\begin{tabular}{|c|c|}
\hline $\mathrm{K}_{\mathrm{rr}}$ & Growth rate of the customer interruption cost \\
\hline $\mathrm{K}_{\mathrm{BPR}}$ & Battery power replacement cost, in $\$ / M W$ \\
\hline $\mathrm{K}_{\mathrm{mf0}}$ & Initial feeder maintenance cost, in $\$$ \\
\hline $\mathrm{K}_{\mathrm{mf}}$ & Growth rate of the feeder maintenance cost \\
\hline $\mathrm{K}_{\mathrm{bf}}$ & Maintenance cost constant of BESS \\
\hline $\mathrm{K}_{\mathrm{bv}}$ & Variable maintenance cost of BESS \\
\hline $\mathrm{K}_{\mathrm{P}}$ & Round trip efficiency \\
\hline $\mathrm{K}_{\mathrm{E}}$ & Charging/discharging efficiency \\
\hline NB & Number of buses \\
\hline $\mathrm{NC}_{1}$ & Number of circuits in feeder sections \\
\hline NH & Number of time zones \\
\hline NL & Number of branches \\
\hline NR & Number of battery replacements \\
\hline NX & Number of batteries \\
\hline NY & Index for the year \\
\hline PV & Photovoltaic \\
\hline $\mathrm{r}$ & Interest rate \\
\hline $\mathrm{PB}_{\mathrm{i}}$ & Power supplied from the battery at bus ' $\mathrm{i}$ ' \\
\hline$\underline{S B}, \overline{S B}$ & Limitation on the generated power of the battery \\
\hline SB & Apparent Power supplied from the battery \\
\hline $\mathrm{SD}_{\text {it }}$ & Apparent power demand at bus ' $\mathrm{i}$ ' \\
\hline$S F E$ & Feeder emergency rating, in MVA \\
\hline$\overline{S F}$ & Peak flow in MVA \\
\hline
\end{tabular}


Apparent power generation

$\underline{S G}, \overline{S G}$

Limitation on the apparent power generated

SL

Apparent power injected from bus into the connected line

$\mathrm{V} \angle \delta$

Vector of the bus voltage phasors

$\underline{V}, \bar{V}$

Acceptable minimum and maximum bus voltages, respectively

$\mathrm{y}_{\mathrm{l}} \angle \theta_{\mathrm{l}}$

Vector of the line admittance

$\mathrm{y}_{11}$

Upgraded vector of the line admittance

\section{Fuzzy Variables}

$\mu_{\mathrm{c}}$

$\mu_{\mathrm{obj}}$

$\mu_{\mathrm{s}}$

$S D(x)$

$\underline{S D}, \overline{S D}$

$\mathrm{TC}(\mathrm{x})$

$\underline{T C}, \overline{T C}$
Fuzzy membership function of cost

Fuzzy membership function of objective

Fuzzy membership function of SAIDI

\section{Complementarity Variables}

$\Psi \mathrm{M}$

$\Psi \mathrm{M}_{\mathrm{i}}, \Psi \mathrm{M}_{\mathrm{j}}$

M

$\mathrm{N}$
AIDI - System Average Interruption Duration Index

Minimum and Maximum of SAIDI

Total Cost Variable

Minimum and Maximum of Total Cost 


\begin{tabular}{|c|c|}
\hline $\mathrm{N}_{\mathrm{S}}$ & Number of loops \\
\hline $\mathrm{N}_{\mathrm{S}}(\mathrm{s})$ & Set of line indices comprising the $s^{\text {th }}$ loop \\
\hline$P D$ and $Q D$ & vectors of bus-wise active and reactive power loads \\
\hline PG and QG & vectors of bus-wise active and reactive power generations \\
\hline PL and QL & Active and Reactive Power Injected from Bus into the connected line \\
\hline $\mathrm{P}_{\text {Loss }}$ & Total Active Power Loss of the System \\
\hline $\mathrm{Y}_{\mathrm{B}}(\mathrm{Y}-\mathrm{BUS})$ & System Admittance Matrix \\
\hline$[\mathrm{YI}]$ & Bus Incidence Matrix \\
\hline$[\mathrm{YL}]$ & Diagonal Matrix of Line Admittance Values \\
\hline \multicolumn{2}{|c|}{ Variables used in Appendix } \\
\hline$a$ & Discount Factor \\
\hline$A N$ & Annuity \\
\hline$P W$ & Present Worth \\
\hline$R N$ & Random Number \\
\hline
\end{tabular}





\section{Chapter 1}

\section{INTRODUCTION}

The major components of an electric power system are generation, bulk transmission system, transmission system stations, distribution system, distribution system substations and primary feeders. The transmission system consists of a network of three-phase transmission lines, transformers, shunt and series capacitors and transmission substations. Typical transmission voltages range from $115 \mathrm{kV}$ up to $765 \mathrm{kV}$. The distribution system consists of step-down transformers, substations, switching capacitors, voltage regulators, and distribution system lines. Typical distribution system voltages range from $4 \mathrm{kV}$ to $69 \mathrm{kV}$.

While this arrangement and configuration is typical, every power system design and implementation arises from requirements of load profile, geography, available energy resources, investments, planning philosophy, operational philosophy, etc. This is specific to each case.

Each distribution substation will serve one or more primary feeders. The feeders are usually radial, with the exception where the ring topology is used for higher reliability. A radial topology means that there is only one path for power to flow from the distribution substation to users or vice versa [1], Fig 1.1.

A typical distribution system will be composed of one or more distribution substations consisting of one or more feeders. Components of the feeder may consist of the following:

- Main Feeder

- Bus

- Transformers 
- Shunt Capacitor Banks

- Switches

- Voltage Regulators

- Protection Devices

Feeders are usually segregated into several sections connected through switches and reclosers. Each feeder section may have several lines connected to it through switches. Feeders run along streets, mostly as overhead lines, and supply distribution transformers that step the voltage down to the secondary distribution level (120 to $480 \mathrm{~V})$. From these transformers, energy flows through secondary mains and services conductors to supply power directly to customer loads. In some cases, underground feeders are used for power distribution where it is difficult to use an overhead system. In congested areas such as cities and towns, the cables are laid below the ground surface. Though the initial investment is significantly more compared to that of overhead lines, the maintenance required is much less compared to the overhead system. The higher cost of underground feeders is mostly due to the high cost of trenching, conduits, cables, manholes and other special equipment. There are disadvantages to using underground feeders in distribution systems. Even though there is only a rare chance of faults occurring in an underground system, it is very difficult to locate the fault point and its repair is difficult and expensive. The more important issue with underground feeders is that expansion for new loads is not possible and can only be met by installation of new lines. For the severely congested cities in North America, these disadvantages are going to be an enormous planning and operating concern, especially with the increase in popularity of Electric Vehicles (EV) and renewable energy sources. 
The penetration of electric vehicles, energy storage and renewable sources such as wind and solar into distribution systems has been growing steadily in recent years and is anticipated to grow at a fast pace in the future. In contrast, in the past, distribution systems were largely passive with only loads. These changes are transforming distribution systems to be active. Active loads, renewable generation and electric mobility have large economic implications and hence it makes it imperative that distribution systems function with a high reliability. In the past, only transmission systems required a high degree of reliability as they were generation connected. Slowly, but steadily, distribution system utilities are embracing smart grid technologies to make their systems more reliable, cost-effective and green.

Consequently, major efforts have been devoted to developing reliable and cost-effective operation of distribution systems (DS). The Electric energy distribution business entails sourcing electric energy from transmission stations and distributing it to customers in the most cost-effective and reliable manner while minimizing capital and operational costs. Accordingly, utilities look to minimize the sum of annual amortized capital costs and annual operating costs per unit of delivered energy, while meeting required reliability of service and quality of supply standards.

It is observed that as the ratio of peak to off-peak power flow increases, total operating and capital costs also increase. This is accentuated with renewables that drive off-peak power flow to a very low value.

One of the recent solutions to increase reliability and increase cost-effectiveness of distribution systems is to use Energy Storage (ES). That is due to the technological advances that enable ESS units to offer several services and a decrease in their prices. The cost of ESS is likely to decrease even more over the coming years to make them more attractive for use in distribution systems. Energy storage can also play a vital role in enabling distribution systems to serve electricity to 
Electric Vehicles (EV), which creates issues due to sudden load growth in concentrated areas and peaky load profiles. Further, ESS units could be used to increase the profit margins of wind and solar farm owners by providing arbitrage type services. In some cases, a hybrid solution of new feeders and Energy Storage Systems (ESS) may cost less than just using new feeders.
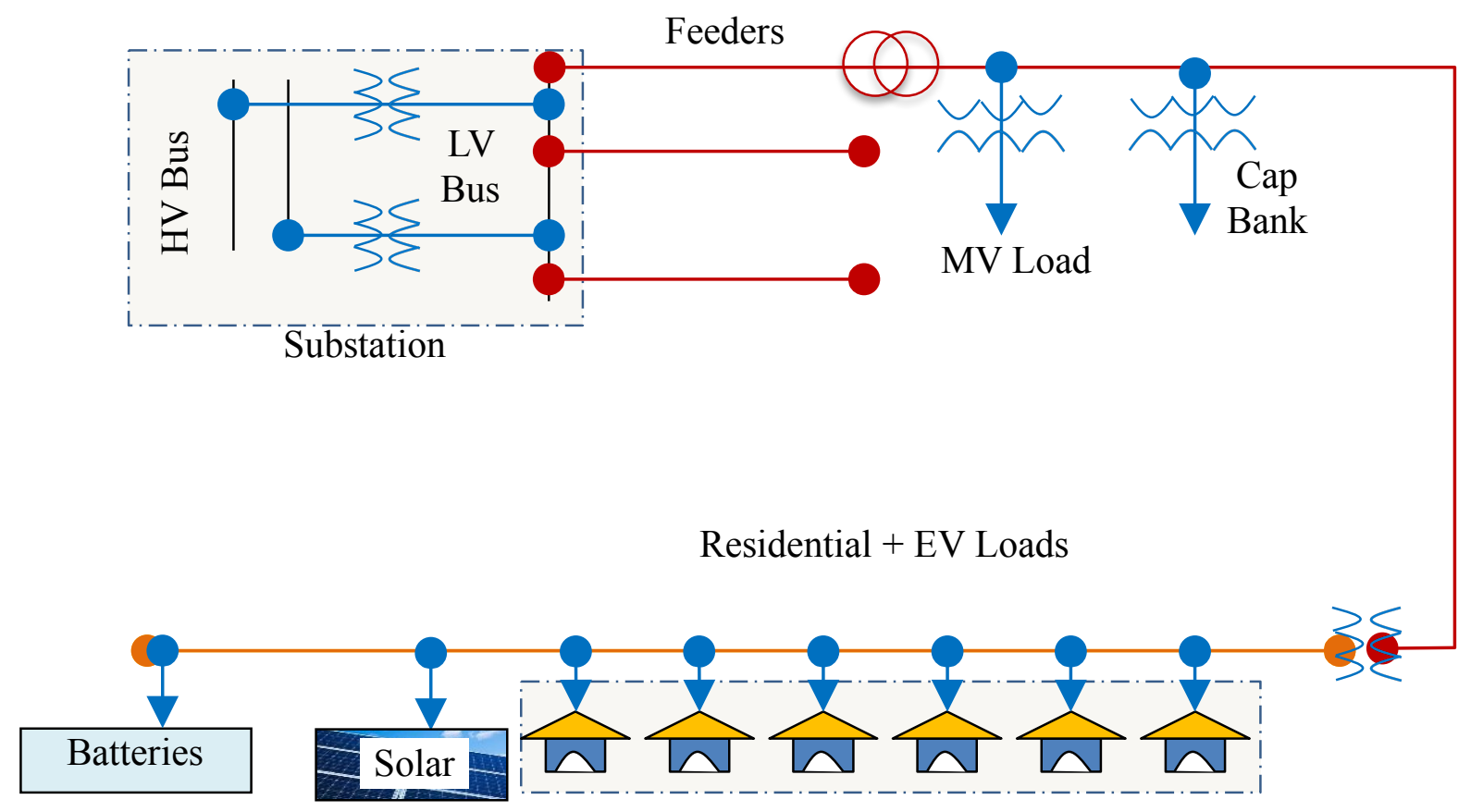

Fig. 1.1 Distribution System in the Presence of Renewables, EVs and ESSs 


\subsection{Effect of Renewables and Electric Vehicles on Load Profiles}

Electric vehicles and renewable generators have typical electricity consumption patterns. Taking an extreme case, electric cars such as Tesla with fast chargers can charge in 30 minutes, taking up to $60 \mathrm{kWh}$ of energy. It equals about $120 \mathrm{~kW}$ power requirement. Contrasting this need with a $2 \mathrm{~kW}$ peak demand for a home in Ontario with Natural Gas heating and $18 \mathrm{~kW}$ peak demand for a home with electric base board type heating, EVs of this type represent a high peak for a very short duration. To understand the effect of renewables and EVs on the load profile of DS, an example is shown that considers a three-bus electrical distribution system with an energy storage device as shown in Fig.1.2. Bus \#1 is the substation. The two loads, Load \#1 and Load \#2, are connected at Bus \#2 and Bus \#3 respectively. In general, the load profile in a day may vary as shown in Fig. 1.3 where $\mathrm{P}_{\text {avg }}$ is the

average demand at the substation in a day considering load and connected generation. $\mathrm{P}_{\max }$ is the peak substation demand in a day. The ratio of $\mathrm{P}_{\mathrm{avg}} / \mathrm{P}_{\max }$ quantifies the utilization factor.

and is the ratio which significantly influences the opportunity for energy arbitrage using batteries. In a peaky load profile, the utilization factor is low and vice versa. 


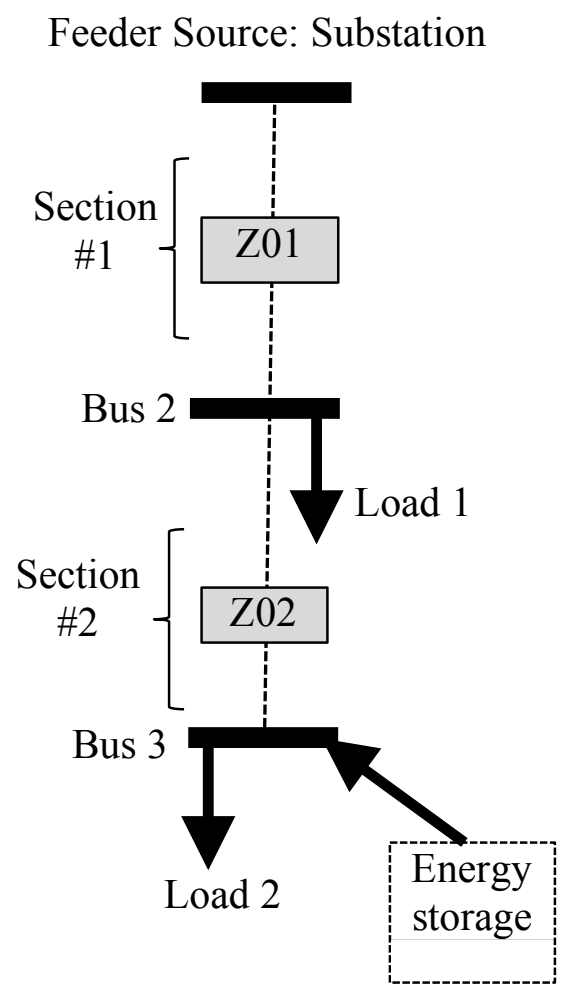

Fig. 1.2 Sample System with Energy Storage System

Ordinarily, without Energy Storage (ES), feeder size should be equal to or more than $\mathrm{P}_{\max }$ to satisfactorily supply connected demand on the feeder. If Energy Storage is connected, it will benefit from arbitrage opportunity, which is the shaded region. This shaded region is a function of $\mathrm{P}_{\text {avg }}$ and $\mathrm{P}_{\max }$.

In fact, if $\mathrm{P}_{\text {avg }}$ is close to $\mathrm{P}_{\max }$, there is no energy arbitrage opportunity and there will be no use of Energy Storage. In such a case, the feeder needs to be sized to equal or greater than $\mathrm{P}_{\max }$. 


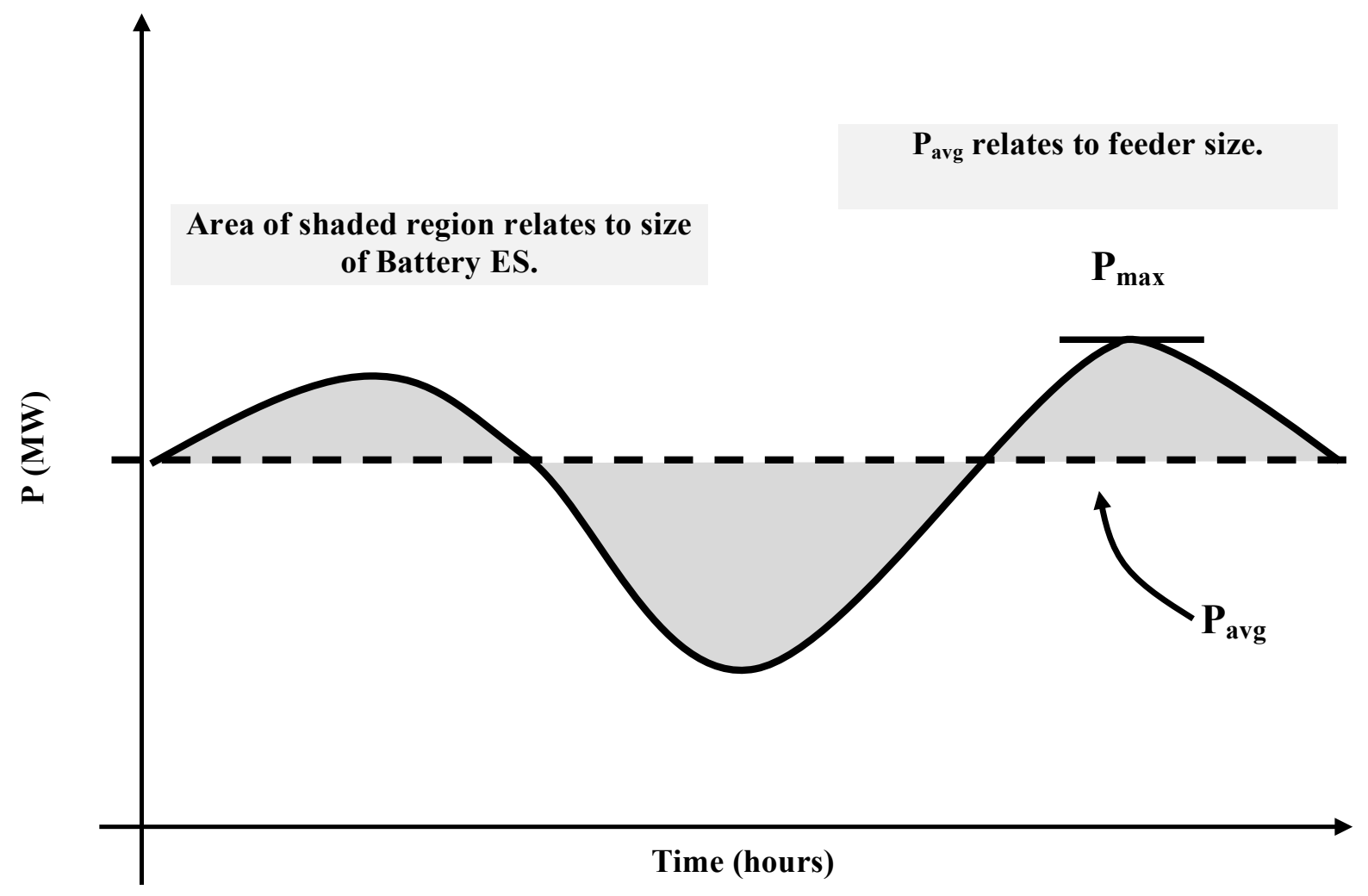

Fig. 1.3 Load Profile of a Day

As the ratio $\mathrm{P}_{\text {avg }} / \mathrm{P}_{\max }$ reduces (that is, as $\mathrm{P}_{\max }$ gets larger), the shaded region in Fig. 1.3 will increase. If Energy Storage is considered, a large sized Energy Storage Unit will be required and the feeder size will be reduced to $\mathrm{P}_{\text {avg }}$. Hence, it may be concluded that the area under the $\mathrm{P}_{\text {avg }}$ line determines the feeder size. The shaded region will determine the magnitude of the energy arbitrage opportunity. The sizes of batteries and feeders also depend on load patterns.

There is an opportunity to consider two options, (1) only feeders of the size $\mathrm{P}_{\max }$, and, (2) feeders of size $\mathrm{P}_{\text {avg }}$ and energy storage units that have a capacity to store and supply the shaded region. 
Hence, if the cost of energy storage is significantly low and feeders have a low utilization factor $\left(\mathrm{P}_{\text {avg }} / \mathrm{P}_{\max }\right)$, then an economic case may be made to install energy storage with lower feeder capacities.

\subsection{Opportunity to Plan with ES as a Cost-Effective Solution}

Good voltage regulation is the most important factor in a distribution system for delivering quality service to consumers. For this purpose, careful consideration is required for the design of new feeders and distribution networks. Further, in the last two decades, distributed generations at low voltage have become pervasive. The rate of transition to $\mathrm{EV}$ is on the rise. These changes have transformed distribution systems to be active with load, generation and energy storage in contrast to the past, where distribution systems were largely passive or load consuming entities. These factors require a robust distribution system with a high degree of reliability. Accordingly, planners are working to evolve newer planning tools. Distribution planning has recently returned to a golden age, with researchers and engineers challenged to develop planning techniques suitable to current and future distribution systems. [2]

Today's electricity delivery system provides high quality and highly reliable power to customers at the least cost. More than a century of development has led to a large, interconnected system that brings power from central station generators via transmission and distribution to customers.

Renewable energy sources at the distribution level are encouraged as they reduce pollution and are pervasive. Connecting new renewables to feeders may require feeder upgrades depending upon the situation. The usual process for utilities is to minimize the cost for feeder and other asset upgrades such that the resulting upgraded system can connect new renewable sources while maintaining system performance within standards. The model is called the feeder investment model that accounts for feeder capital costs and maintenance costs accrued via loading. The issues of intermittency and 
increase in system cost could be mitigated by planning to have energy storage solutions at appropriate locations. As battery energy storage systems are now an important part of many renewable energy systems, the selection of battery size also becomes an important task as it has a major influence on the reliability and cost of the system. A large energy storage system would clearly improve the system reliability but is usually not justified due to high overall cost. Therefore, a tradeoff always exists between reliability and cost. This project deals with deployment of battery energy storage solutions in distribution networks which have variable loads and renewable energy sources. Through this deployment, a distribution system could schedule and use a set of distributed energy storage units connected to different locations, deferring major capital investments and lowering costs for all customers.

Energy Storage Systems are typically very expensive in capital, operation, and maintenance costs. However, the installation costs of energy storage have declined by almost $50 \%$ in the last decade. They are expected to decrease further in the future.

Meanwhile large distribution utilities recognize that the purpose of their asset planning program is to maximize the value they provide to their stakeholders as measured in terms of its focus on customer service, asset renewal, and consistent financial performance. Asset planning comprises the tools by which utilities evaluate spending proposals to identify those that provide the best value. At its core, asset planning is about evaluation, justification, and prioritization of spending programs. Some utilities such as Toronto Hydro-Electric System Limited (THESL) are constantly looking for new methods to operate and move forward from the traditional techniques. In fact, they are on the path to becoming an industry-leading asset management utility. Their adoption of the feeder investment model is evidence of such leadership.

Basically, the Feeder Investment Model (FIM) is a risk-based economic model for identifying the 
economically optimal intervention timing for aging assets. This model considers all the costs associated with the system and does life cycle analysis. The expected outputs from this model would be the optimal replacement time for assets and the minimum life cycle cost. When used with renewable resources, energy storage can increase their usability of photovoltaic and wind generated electricity by making this generation coincident with peak load demand. The energy storage devices can store power/energy during off-peak periods and discharge it during peak load periods. This enhances the utilization of network infrastructure, resulting in the deferment of system upgrades to later years, which may reduce the feeder investment cost. In this work, the proposed FIM is constructed by also considering energy storage devices.

\subsection{Current DS PlanNing Methods}

To meet their business needs, distribution utilities must develop the least costly asset solution possible. On the other hand, a battery energy storage system (BESS) is a useful method of enabling the incorporation of renewable resources and electric transit. However, current asset planning methods employed by distribution utilities fail to include BESS as a possible asset.

The distribution system asset (DSA) planning algorithms evaluate a variety of possibilities and then select the least costly asset upgrade option. Despite the sophistication of these DSA planning algorithms and their outstanding performance, the advent of new smart grid tools such as microgrids and energy storage systems has resulted in a need to explore new algorithms and DSA planning paradigms. Connection of new renewables and electric vehicles to distribution systems in the future will require feeder upgrades. The common practice is for utilities to minimize their costs for feeder and other asset upgrades, so that the resulting upgraded system is able to handle the connection of new renewable sources and electric vehicles, while maintaining system performance within 
applicable standards. Meeting this goal requires careful consideration of the design of new feeders and distribution systems [3].

The main component of the DSA planning methods used by several utilities is the so-called Feeder Investment Model (FIM), which is a risk-based economic model for identifying the economically optimal plan for incorporating new assets and replacing aging ones. For fair comparison of the cost of various plan options, the practice is to consider the annualized costs of new and replacement assets (feeders, transformers, etc.), operation and maintenance, and customer interruptions over a planning period that reflects the current year. The resulting new or upgraded distribution system must connect new loads and/or generation while satisfying performance standards at the lowest annualized cost [4]. As shown in Fig. 1.4, the optimization must be based on the minimization of two competing cost curves: the annualized capital cost and the annualized risk cost. The annualized risk cost includes the cost of customer interruptions as well as the costs of operation and maintenance. As one can see from these curves, the risk cost increases when the year of intervention increases, as the probability of fails increases when the investment is postponed. On the other hand, the capital cost decreases when the year of intervention increases, as the investment is postponed.

Presently, the optimal asset planning algorithms used by utilities do not consider the use of battery energy storage systems and the current objective function of their algorithm looks like the following:

$$
f_{S F}(\overline{S F}, N Y)+f_{m c F}(N Y)+f_{C I C}(S F E, \overline{S F}, N Y)
$$

where $f_{S F}$ is capital cost of feeders, $f_{m c F}$ is maintenance cost of feeders and $f_{C I C}$ represents the customer interruption costs. 


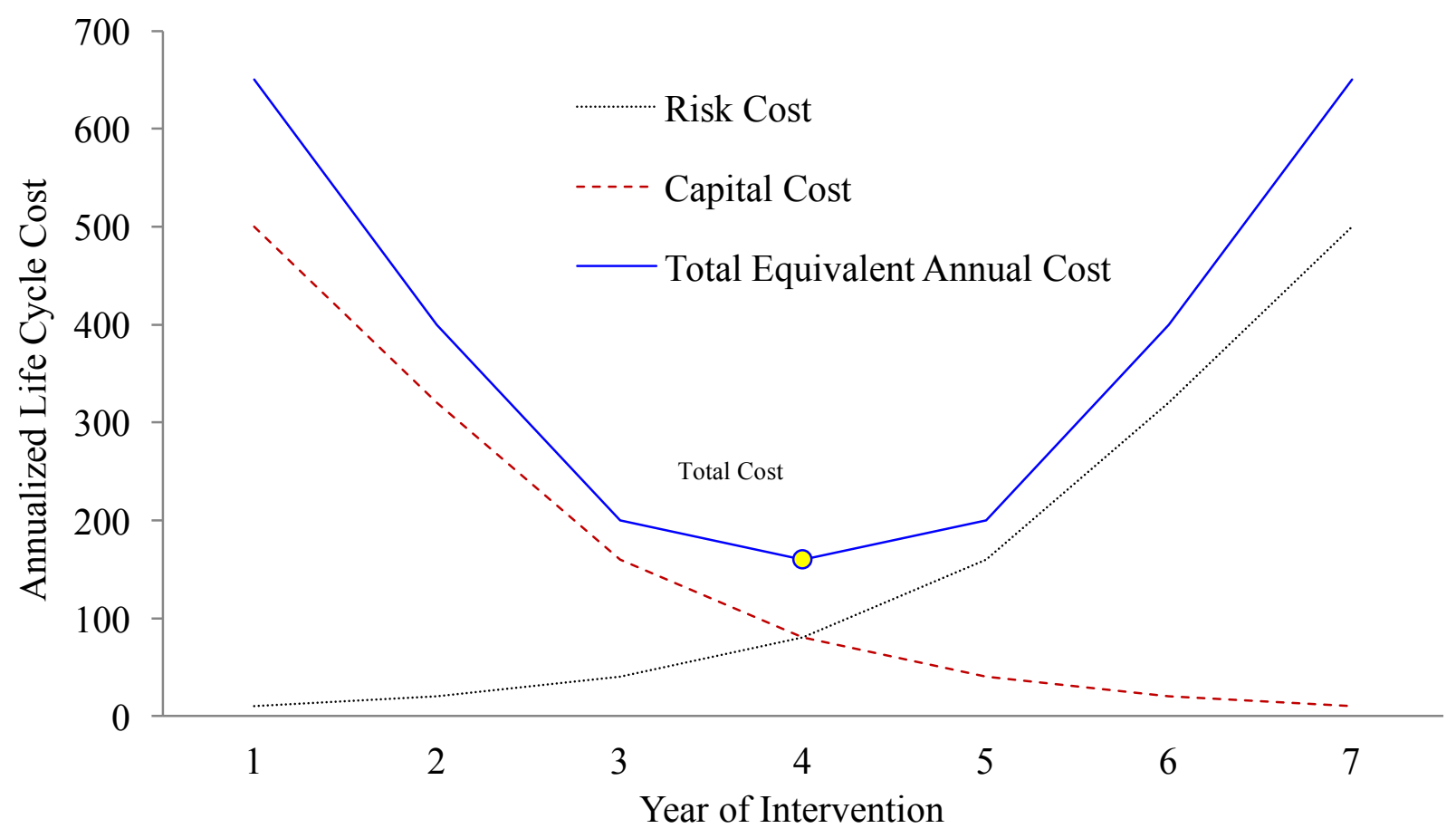

Fig. 1.4 Representation of the Equivalent Annual Costs

\subsection{ReCENT DeVELOPMENTS OF DS PLANNING WITH ES}

Renewable energy sources, such as wind and solar, have large potential to replace conventional fossil fuels and to reduce greenhouse gas emissions worldwide. However, both solar photovoltaic (PV) and wind electric generations, which have uncertainty in nature, may cause large reverse power flows, especially during off-peaks, from where they are installed back to transformer stations. Besides, with rapid development in electric vehicles (EV), there is potentially a large amount of demand that will appear in distribution systems. These new generation sources and demands will heavily increase the operational burden of distribution systems. Utilities are seeking proper tools to upgrade their distribution systems to adopt more renewables and to supply higher demand. 
In traditional ways, transformers and lines (major components of a feeder) are added on feeders to supply additional demand. These asset additions are usually very expensive. With recent advancements in energy storage technologies, battery energy storage systems (BESS) appear to be an option for utilities to consider in their system upgrades. California seeks to install $186 \mathrm{GWh} / 22$ GW of BESS to enable its $85 \%$ penetration of renewable generation [28]. The Northwest Power Pool will need up to $10 \mathrm{GWh} / 1 \mathrm{GW}$ of BESS by 2019 to balance $14.4 \mathrm{GW}$ of installed wind generation [29]. However, choosing locations and sizes of BESS in distribution networks is still a challenge.

The literature includes proposals for the sizing of energy storage (ES) in the context of active distribution systems and distributed generation (DG), but the methods suggested are based mainly on the adoption of trial-and-error approaches. In [5] - [7], this approach involves the investigation of a number of predetermined ES sizes and consideration of a variety of control schemes. The optimal size is achieved based on the existence of the correct level of trade-off between the cost of the ES facility and the operational costs [5], [6] or the volume of curtailment [7]. Although trialand-error approaches may be feasible and practical, given the existing combinations of power and energy capacities, they cannot encompass the exploration of a large search space. Adequately covering such a search space becomes even more challenging when multiple ES units are considered simultaneously. The research in [8] discusses the reduction of curtailment of renewable distributed generation (DG), specifically wind farms, simultaneously with the management of congestion and voltages through the introduction of a planning framework for determining the minimum storage sizes (power and energy) at multiple locations in distribution networks. However, this study fails to consider DSA planning or the economics of asset planning. 
The authors of [9] presented an active-reactive optimal power flow (A-R-OPF) for distribution systems with embedded wind generation and battery units based on a fixed-length charge and discharge cycle for the daily operation of battery units. In a further study [10], the same authors discussed a flexible battery management system whereby higher profits could be achieved than with a fixed-operation strategy for the use of battery units. This topic was also explored in [11], with the goal of ensuring both practical and optimal operational strategies, which was accomplished based on consideration of a flexible A-R-OPF with a flexible on-load-tap-changer (OLTC) control system. According to [12], the unique advantages of renewable DG sources have led to their increased penetration in active distribution networks. However, the incorporation of non-dispatchable DGs such as wind turbines raises questions about the risk of uncertainty and power quality issues within distribution networks. Such problems could be eliminated by the appropriate application of ES units, and in fact, a method for optimizing the allocation and economic operation of ES devices in a lowvoltage microgrid system was presented in [13]. In [14], the authors investigated the use of a genetic algorithm (GA) optimization technique to solve a multiple objective function for evaluating the economic impact of energy-storage-specific costs on the net present value of ES installations in distribution substations. To minimize the costs of power system assets and improve system voltage profiles through the siting and sizing of storage units, while including consideration of the uncertainty associated with wind power production, a hybrid multi-objective particle swarm optimization (HMOPSO) approach was proposed in [15]. The authors of [16] examined methods for determining optimal energy storage system operation to increase the value of wind-power generation. In [17], the researchers presented a probabilistic approach for ES sizing and siting in distribution systems in order to improve the reliability of distribution systems. Their algorithm is dependent on load shedding and it does not compare the cost of ES with the cost of upgrading other assets. 
Additional research, such as the work presented in [18] and [19], has been focused on sizing ES units for isolated microgrid applications. The optimization of the cost and arbitrage benefits of ES installation was discussed in [20] with respect to the costs of system upgrades and energy losses. The primary goal was to determine the optimal size, location, and operation of the storage units to be installed, so that the total system costs would be minimized and system benefits maximized. The work was based on the use of a combination of linear programming and a GA, coupled with a fixed planning period problem. Therefore, the algorithm was not designed to look for the optimal number of useful years for the assets and instead uses an assumed number to base the search for a solution to the planning problem. The methods proposed in [18] - [20] do not consider green field systems where there is no existing structure and in cases where the system requires additional feeder upgrades, it fails to account for the added feeder in the power balance equations. In [21], the authors discuss future demands on the grid structure. They claimed that a simple answer will be insufficient for addressing the operation of new smart technologies and that planning and operation processes must also be adapted to the new demands. Their conclusion was that the integration of decentralized energy resources in distribution networks extends beyond technical questions and requires a holistic integrative approach on a variety of levels.

The study presented in [22] investigates how information from vulnerability analyses and existing maintenance management systems can be combined with details about threats to establish indicators of vulnerability in power lines.

Much work has been done on the sizing and siting of BESS in distribution systems, and numerous applications of BESS are considered. These applications, such as energy arbitrage in transmission systems [25], power flow optimization with peak shaving [26], frequency [27] and voltage [28] support, as well as overcrowding management [25] - [29] have been studied. Sizing BESS in the 
context of active distribution systems with distributed generators (DG) is proposed in [30]. Tools are developed to optimally size a set of BESS with prediction errors in the forecast of the renewable sources.

In addition to sizing BESS, work was done to alleviate the detrimental impacts of short-term wind power and load fluctuations [31] and high penetration PV [32] in distribution systems. Capacity expansion of BESS through the time span that can result in significant cost saving is illustrated in [33] and [34]. Factors such as reliability criteria together with the investment and the operation costs are considered. A bilevel program that determines the optimal location and size of storage devices to perform the spatiotemporal energy arbitrage is developed in [35]. This method aims to simultaneously reduce the system-wide operating cost and the cost of investments in BESS while ensuring that merchant storage devices collect sufficient profits to fully recover their investment cost. The research in [36] to [40] discusses advantages and other aspects of BESS. However, none of the above literature considers the impact of BESS on distribution system reliability.

This review of the literature thus reveals that several methods have been devised for developing an optimal plan for BESS in distribution systems. However, none of these methods integrates BESS into the distribution system asset planning algorithms in a way that allows utilities to evenly compare several plan options with and without BESS based on an evaluation of the annualized costs of the asset plans. 


\subsection{BeSS Model Used in Planning And Evolution of Battery Technology}

Energy storage systems can be used in distribution systems by utilities for several applications. Some

of these applications and advantages are as below:

- Enabling the integration of renewable energy sources;

- Improving the reliability of distribution systems by providing back up power;

- Deferring the upgrade of assets in distribution systems;

It should be mentioned that the choice of a certain energy storage technology over others, is mostly dependent on the application and economics of the particular situation. In this study, the goal is planning of DS using ESS, which means improving the reliability of distribution systems, and deferring the expensive asset upgrades.

For this purpose, batteries are selected as the candidate storage technologies since their power and discharge time capacities are suitable for the application under study. The capacity model of Lithium-Ion batteries treats the battery as a tank of charge, removing and adding charge as needed, assuming that positive power implies discharging from the battery. The battery is only allowed to discharge to the user-specified minimum state-of-charge and stays within user-specified rates of current charge and discharge. Capacity relates to battery energy through the voltage. Power relates to energy by calculating how much energy is transferred over a period of time.

In this dissertation, the model of battery energy storage is from a planning perspective. Hence, simple models are used that consider power conversion system efficiency and self-discharge on an 
hourly basis. Transient, electro-chemical and thermal modeling is not a part of this dissertation. A simple schematic is provided in Fig. 1.5 for reference.

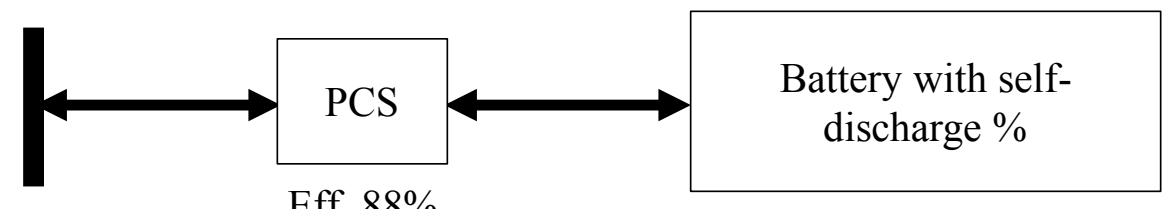

Bus

Eff. $88 \%$

Fig. 1.5 Battery Power Conversion System Efficiency and Self-Discharge Schematic

The lithium-ion battery technology is been around for around half a century and has taken over the electronics industry and is on the verge of entering the transportation industry and the utility grid. With the advancement of technology in manufacturing of batteries, it is safe to predict a massive drop in the battery prices in the near future, Fig. 1.6.

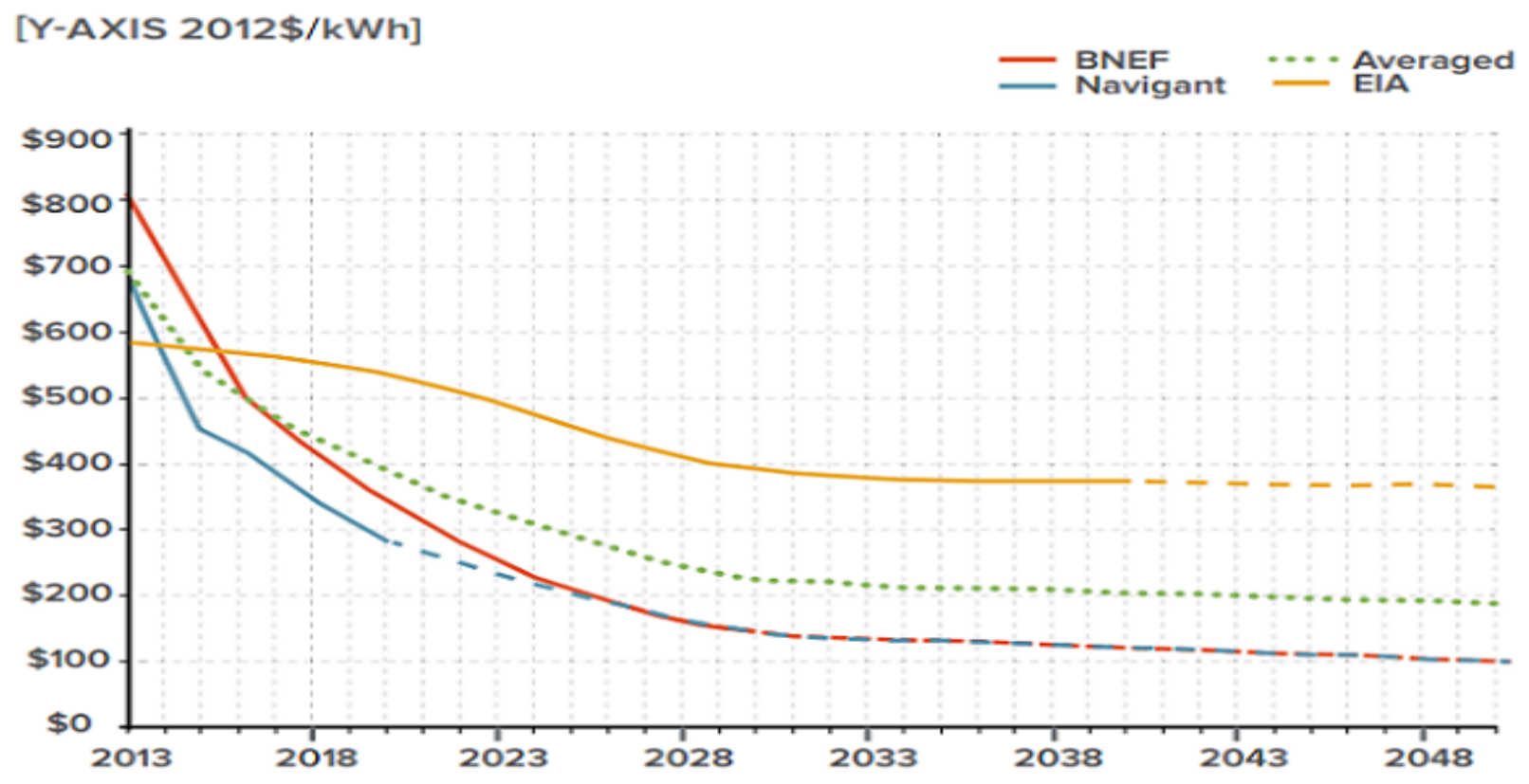

Fig. 1.6 Battery Price Prediction [74] 


\subsection{Opportunity to ReConfigure And Improve System Performance}

Smart Grid technologies enable reconfiguration of distribution systems in real-time to enable improved performance and reduced losses. With increase in EVs and renewables, and them causing a peaky load profile, it is the best to use the concept of reconfiguration in real-time to best operate distribution systems. Feeder reconfiguration can be used to solve different objectives. For example, the service restoration to disconnected areas and/or the minimization of active power losses. Therefore, applying a reconfiguration could provide solutions for real-time emergency situations as well as increase reliability and power quality.

Since reconfiguration involves switching of circuits, it is a mixed integer nonlinear optimization challenge. It has a set of solution spaces which are nonlinear in nature. Typically, reconfiguration is a complicated problem to solve, since the number of solution iterations grow exponentially with the number of available tie switches, leading to a time consuming, very difficult mathematical computation effort to achieve the optimal configuration.

The analysis of a distribution feeder is important to an engineer in order to determine the existing operating conditions of a feeder, and to be able to consider "what if" scenarios of future changes to the feeder. For example, it is essential to know the location and status (i.e. on/off) of shunt capacitors and their reactive power rating. Shunt capacitor banks including fixed and switched banks are used on primary feeders to reduce voltage drop, reduce power losses, and improve power factors. Capacitors are typically switched off during the night for light loads and switched on during the day for heavy loads.

In the case of existing switches, besides their locations, it is important to know their normal open/close status. To reduce the duration of interruptions due to transient faults, such as lightning, overhead feeders can be protected by automatic reclosing devices. [41] 
To further reduce the duration and extent of customer interruptions, switches are installed at selected intervals along radial feeders. In the case of a permanent fault, one or more switches open to isolate the fault. In addition, normally open tie switches to adjacent feeders are incorporated, so that during emergencies, healthy sections of a feeder can be tied to the adjacent feeder supplied by a different source. Spare capacity is often allocated to feeders to prevent overloads during such emergencies. Many utilities have also installed automatic fault locating equipment and remote control switches at intervals along radial lines, so that faulted sections of a feeder can be isolated and healthy sections reenergized rapidly from a dispatch center [42] - [43].

As it mentions before, primary distribution includes two basic systems:

- Radial

- Mesh (i.e. interconnected)

The mesh system is used where high service reliability is important. The feeder loops around a load area and returns to the distribution substation, especially providing two-way feed from the substation. The size of the feeder conductors, which are kept the same through the ring, is usually selected to carry the entire load connected to the loop, including future load growth. Then switches are used to reduce customer interruptions which are the focus of this study. The ring is normally operated with the open switch and power to a customer at any one time is supplied through a single path from the distribution substation.

\subsection{Current Optimal Reconfiguration Methods}

Optimization theory and algorithms play a key role in reconfiguration problems. Many optimization methods have been used to make the problem of reconfiguration more reliable and faster. However, 
all these methods were limited to Heuristic and Genetic algorithms due to the open/close nature of switches which turns the problem into a mixed integer algorithm.

\subsubsection{Heuristic Methods and Genetic Algorithm}

The evolution usually starts from a population of randomly generated individuals and is an iterative process. The population in each iteration is called a generation. In each generation, the fitness of every individual in the population is evaluated; the fitness is usually the value of the objective function in the optimization problem being solved. The more fit individuals are stochastically selected from the current population, and each individual's genome is modified (recombined and possibly randomly mutated) to form a new generation. The new generation of candidate solutions is then used in the next iteration of the algorithm. Commonly, the algorithm terminates when either a maximum number of generations has been produced, or a satisfactory fitness level has been reached for the population.

Initially many individual solutions are (usually) randomly generated to form an initial population. The population size depends on the nature of the problem, but typically contains several hundreds or thousands of possible solutions. Traditionally, the population is generated randomly, allowing the entire range of possible solutions (the search space). Occasionally, the solutions may be "seeded" in areas where optimal solutions are likely to be found. This generational process is repeated until a termination condition has been reached. Common terminating conditions are:

- A solution is found that satisfies minimum criteria

- A fixed number of generations is reached

- The allocated budget (computation time/money) is reached

- The highest-ranking solution's fitness is reaching or has reached a plateau such that successive iterations no longer produce better results 


\subsubsection{Nonlinear Optimization versus Genetic Algorithms}

Although all the referred papers have found optimal solutions, all of them were forced to use intelligent algorithms due to the zero-one state nature of switches. The discontinuous nature of solution space deprives benefits of a typical optimal power flow algorithm. Some of the disadvantages of using these kinds of intelligent techniques are explained in [44] and [45]:

- Repeated fitness function evaluation for complex problems is often the most prohibitive and limiting segment of artificial evolutionary algorithms. Finding the optimal solution to complex high dimensional, multimodal problems often requires very expensive fitness function evaluations. In real world problems such as structural optimization problems, a single function evaluation may require several hours to several days of complete simulation. Typical optimization methods cannot deal with such types of problem. In this case, it may be necessary to forgo an exact evaluation and use an approximated fitness that is computationally efficient. It is apparent that amalgamation of approximate models may be one of the most promising approaches to convincingly use GA to solve complex real-life problems.

- Genetic algorithms do not scale well with complexity. That is, where the number of elements which are exposed to mutation is large there is often an exponential increase in search space size.

- The "better" solution is only in comparison to other solutions. As a result, the stop criterion is not clear in every problem.

- In many problems, GAs may tend to converge towards local optima or even arbitrary points rather than the global optimum of the problem. This means that it does not "know how" to sacrifice short-term fitness to gain longer-term fitness. The likelihood of this occurring 
depends on the shape of the fitness landscape: certain problems may provide an easy ascent towards a global optimum; others may make it easier for the function to find the local optima.

- GAs cannot effectively solve problems in which the only fitness measure is a single right/wrong measure (like decision problems), as there is no way to converge on the solution. These disadvantages limit the practicality of these intelligence-based techniques in real-time applications. In general, evolutionary algorithm and artificial intelligence techniques are useful only when no other classical method is available.

\subsection{ReCEnt DeVelopments of DS Reconfiguration Methods}

Smart radial distribution system of the future will provide improved performance, reliability, and flexibility to best connect new elements such as renewable generators, energy storage, electric vehicles, etc. smart radial distribution system using a distribution management system (DMS) will be able to optimally reconfigure the system in real-time to improve performance and provide a highly reliable service.In a future setting, smart radial distribution system would have breakers and switches that are remotely controlled via DMS taking optimal settings from the optimal reconfiguration algorithm. It is necessary to have optimal reconfiguration algorithms that can function in real-time to benefit from the smart radial distribution system infrastructure. It is also necessary to develop optimal reconfiguration algorithms that are reliable and efficient which do not depend upon heuristics.

Electric utilities may reconfigure their network such that total system losses are minimized and voltage profile is satisfactory.

Optimal reconfiguration requires that one breaker or tie switch in every loop is opened, such that the set of closed breakers result in a radial topology which results in minimum losses and a satisfactory 
voltage profile. An optimization algorithm for optimal reconfiguration shall consider all possible combinations and scenarios of where one switch can be opened in every loop. This problem is a combinatorial challenge and its complexity increases exponentially with the number of switches. Further, examining the solution domain, which is undertaken in the next section, it is evident that the solution space is discontinuous.

Literature review shows that many researchers have attempted different optimization techniques and have found the optimal solution for published examples. However, limitations in classical linear and nonlinear optimization techniques preclude them from being successfully used for this optimization challenge. Hitherto, all attempts are restricted to optimizing using heuristic techniques such as evolutionary programming, genetic algorithms, etc.

Several methods [46] to [64] have been utilized in an effort to solve this mixed-integer non-linear program using genetic algorithms. The research in [46] and [47] has been successful in finding an optimal solution using fuzzy genetic and harmony search algorithms. A fuzzy controlled real coded genetic algorithm was used in [48] to solve the reconfiguration problem. Two controllers had been used to adaptively adjust the crossover and mutation probabilities based on the fitness function. An efficient codification to solve the distribution network reconfiguration for the loss reduction problem is reported in [49]; however, they are still using evolutionary algorithms and are not benefiting from using a regular NLP. Mendes et al. [50] used evolutionary algorithm to address the particular case of reconfiguration after an outage caused by the loss of a single branch of the network. The work in [51] claims that a multi-objective evolutionary algorithm (EA) based on subpopulation tables adequately models several objectives and constraints, enabling a better exploration of the search space. Research in [52] presents a novel charged system search (CSS) algorithm, the particle moving evaluation mechanism CSS (PMEM-CSS), for determining the switching strategy to solve 
the distribution system loss minimization problems. In [53], a novel optimization method that provides an error bound on the solution quality is found. Thus, the obtained solution quality can be evaluated in comparison to the global optimal solution. Instead of using local updates, a highly compressed search space is constructed using a binary decision diagram, and the optimization problem is reduced to a shortest path-finding problem.

A non-revisiting genetic algorithm $(\mathrm{NrGA})$ is used to determine distribution network configuration for loss reduction [54]. By advocating binary space partitioning (BSP) to divide the search space and employing a novel BSP tree archive to store all the solutions that have been explored before, a new solution is generated by genetic algorithms (GA), and can mutate an alternative unvisited solution. The main contribution of [55] is the presentation of GA with two network encodings, capable of representing only radial connected solutions without demanding a planar topology or any specific genetic operator. In [56], the GA was successfully applied to the loss minimization reconfiguration problem. In the proposed algorithm, strings consist of sectionalizing switch status or radial configurations, and the fitness function represents the total system losses and penalty value for voltage drop and current capacity violations. In [57], an effective approach based on the particle swarm optimization with integer coded is presented to determine the switch operation schemes for feeder reconfiguration. Fuzzy adaptation of evolutionary programming has been also implemented [58]. Ant colony search-based loss minimization for reconfiguration of distribution systems is used in [59]. In [60], the same problem is solved using GA. To enhance its ability to explore the solution space, efficient genetic operators are developed.

The algorithm in [61] is based on a heuristic rule and fuzzy multi-objective approach and it has been tested on a 69-bus system to solve the network reconfiguration problem. While the work reported in literature has found optimal solutions, evolutionary computations and genetic algorithms 
were always used due to the zero-one state nature of switches. The solution space is always discontinuous and does not derive the benefits of a typical optimal power flow algorithm. Some of the disadvantages of using such techniques are explained in [62] and [63]. In research [64], the problem of reconfiguration of distribution systems considering the presence of distributed generation is modeled as a mixed-integer linear programming (MILP) problem. It was possible to create a robust mathematical model that is equivalent to the mixed-integer non-linear programming model, and guarantees convergence to optimality using classical optimization techniques. The routine is tested and validated on real power system.

In summary, there is no guarantee for finding optimal solutions in a finite amount of time when using GA. In addition, parameter tuning is mostly accomplished by trial-and-error and there is no absolute assurance that GA will find a global optimum at all. One of the other disadvantages of GA is that it cannot assure constant optimization response time. Furthermore, the difference between the shortest and longest optimization response time is much larger than with conventional optimization methods. Such properties limit the practicality of these techniques in real-time applications. In general, GA and artificial intelligence techniques are valuable only for offline solutions or when no other classical method is available. Hence, it is evident that use of current optimal reconfiguration algorithms for smart radial distribution system in DMS is not possible and requires new techniques amenable for real-time applications. 


\subsection{Motivation}

\subsubsection{Need for New Methods of DS Planning with ES}

On reviewing the distribution system landscape, it is evident that renewables and electric vehicles will be integrated in a large scale with distribution systems. It is also evident that they will cause a peaky load profile, which leads to a poor utilization factor of distribution system assets.

On reviewing literature on distribution system planning and problems due to renewables and EVs as pointed out in sections 1.1 to 1.5 , it is beneficial to include ES storage into DS planning algorithms and consider ES alongside with feeders and transformers. This is true especially when utilization factors are low for assets with a peaky load profile.

Without existing methods and means for optimal planning of distribution systems, considering feeders and energy storage assets, it is not possible to develop least cost distribution system plans. A new formulation and algorithm will enable distribution system planners to plan for the least cost asset solutions considering ES as well, in addition to regular distribution system assets such as lines and transformers. This is the first motivation for this thesis.

\subsubsection{Need for New Methods of DS Reconfiguration}

New smart grid technologies are enabling real-time control and reconfiguration of distribution system networks. When the loads on adjacent feeders have peaky load profiles and those peaks are not time-coincident, then reconfiguration provides an invaluable method to minimize losses and reduce burden on feeders and other distribution system assets. 
The problem of turning switches on and off is a mixed integer nonlinear optimization challenge. This challenge requires mixed integer nonlinear optimization solvers which are typically not robust. Hence, a lack of conventional real-time mixed integer nonlinear optimization methods precludes use of optimal reconfiguration methods for operational purposes.

This creates the second motivation for this work.

\subsection{OBJECTIVES OF THE THESIS}

With the falling cost of renewables, wind and solar sources are being integrated in enormous amounts into electric grids. At the same time, because of the developments in electric vehicles and the popularity of them, there is an additional load demand that needs to be addressed. Therefore, as outlined in the motivation section, there is an absolute need for innovative algorithms to plan and operate modern distribution systems. For these reasons and motivations, the following objectives have been chosen for this dissertation:

1) A new Feeder Investment Model (FIM) and algorithm will be developed in the context of Distribution System Asset (DSA) planning that takes into consideration Battery Energy Storage Systems (BESS) in addition to conventional assets, such as feeders and transformers. It will consider a distribution system characterized by new and existing variable loads such as electric vehicles and intermittent renewable energy sources. The algorithm is also capable of calculating the optimal number of years for asset use before their replacement is required.

2) Typical feeder investment model considers asset costs in addition to operational and maintenance costs. These operation and maintenance costs consider costs due to SAIDI (system average interruption duration index) and Customer Interruption Costs (CIC). The CIC values are determined via a survey and hence are always an inaccurate value. 
The second objective is to address the conflicting objectives of minimizing asset costs and SAIDI for the system. A new fuzzy logic algorithm will be developed that considers two contradicting objectives in different units. It tries to reduce the asset cost in dollars per year while minimizing the SAIDI measured in minutes per year. In such a case, the SAIDI doesn't need to be translated into a dollar amount using approximate values of CIC. In other words, the algorithm maximizes the reliability while the cost is being minimized at the same time. This method takes into consideration the incorporation of energy storage systems in addition to conventional assets, such as feeders and transformers, into a distribution network characterized by new and existing variable loads and intermittent renewable energy sources.

3) The third objective is to develop a new optimal reconfiguration technique for smart distribution systems using complementarity constraints. The use of complementarity constraints transforms the set of discrete nonlinear solution spaces into one single solution space such that conventional Nonlinear Programming (NLP) techniques can be used for solving the challenge of optimal reconfiguration of Radial Distribution Systems (RDS) in real-time.

\subsection{SUMMARY OF CHAPTERS}

This dissertation is organized in the following chapters:

In Chapter 2, a new DSA planning algorithm that includes the installation and optimal sizing of BESS units in addition to traditional assets, such as feeders and transformers is proposed and demonstrated. It incorporates plan and asset lifetimes as a means of establishing the minimum total annualized costs of new and replacement assets, operation and maintenance, and customer interruptions. The algorithm is successfully validated on 3-bus and 33-bus distribution systems. 
In Chapter 3, a multi-objective FIM planning algorithm with contradicting objectives of System Average Interruption Duration Index (SAIDI) minimization measured in minutes per year and cost minimization measured in dollars per year is proposed. This method is a multi-objective algorithm that uses fuzzy optimization technique to handle multiple contradicting objectives that cannot be combined into a single objective as they are in different units. The objectives are translated into fuzzy satisfaction functions and their minimum is maximized to determine the optimal solution. The proposed algorithm is successfully demonstrated on a 33-bus distribution system.

In Chapter 4, a real-time optimal reconfiguration algorithm which uses a classical nonlinear optimization technique is demonstrated. Using complementarity, the nonlinear mixed integer optimization challenge of optimal reconfiguration with discontinuous solution space is transformed into a nonlinear optimization challenge with continuous solution space. This transformation allows the use of classical nonlinear optimization solvers that are robust, guaranteeing the optimal solution while being suitable for real-time application. This is successfully demonstrated on 7-bus, 33-bus and 69-bus distribution systems and the results are compared with those available in literature with respect to solution time, accuracy in results and robustness of the proposed algorithm and the superiority of the proposed technique has been verified.

In Chapter 5, conclusions and possible future research directions are presented.

\subsection{CONCLUSION}

This chapter introduced the challenge of planning and operating new distribution systems in the presence of a peaky load profile due to growth of EVs and renewables. A literature review was presented for planning techniques when using BESS and also operating techniques through 
reconfiguration. The research objectives were also presented along with chapter wise summary. The results and accomplishments will be presented and discussed in the chapters that follow. 


\section{CHAPTER 2}

\section{A Model for Distribution System Asset Planning}

\section{INCLUDING BATTERY ENERGY STORAGE}

This review of the literature in the previous chapter reveals that several methods have been devised for developing an optimal plan for BESS in distribution systems. However, none of these methods integrates BESS into the distribution system asset planning algorithms in a way that allows utilities to evenly compare several plan options with and without BESS based on an evaluation of the annualized costs of the asset plans.

With the objective of overcoming these limitations, a new Feeder Investment Model (FIM) in the context of Distribution System Asset (DSA) algorithms has been proposed that takes into consideration the incorporation of energy storage systems in addition to conventional assets, such as feeders and transformers, into a distribution network characterized by new and existing variable loads and intermittent renewable energy sources. The algorithm is also capable of calculating the optimal number of years before asset replacement is required.

A further consideration is that load characteristics, such as the load factor, significantly influence the extent of the benefits to be derived from the use of BESS. For the purposes of the work described in this research, the load factor is defined as the ratio between the average demand and the peak demand, which is influenced by the nature of the load and generation patterns of renewable resources connected to the system. 
A final factor is that the inclusion of BESS in a distribution system affects the costs associated with the entire plan, i.e., operation and maintenance costs and customer impact costs. These aspects have been explored in the proposed DSA planning algorithm, which is designed for determining plan life and includes consideration of the costs of both new and end-of-life replacement assets in addition to the costs of operation, maintenance, and customer interruptions. All costs are computed for the entire plan life and reflect those for the present year annualized over the planning period. These annualized planning costs enable a fair comparison of a variety of DSA plans with and without BESS. The proposed DSA planning method is constrained by limits on bus voltages, feeder capacities, BESS unit capacities and bus-power balance equations for the entire distribution system. The method has been tested on a Canadian distribution system feeder that was simplified into a 3bus system and on the IEEE 33-bus system.

\subsection{Problem Formulation of FIM with BESS}

A DS asset plan typically for a greenfield is to minimize the total asset cost to supply forecasted demand while meeting all equipment limits and prevalent standards. Typically, this planning formulation annualizes all cost so that they may be effectively compared.

This section introduces the proposed DSA planning formulation and algorithm that integrates planning for traditional assets, such as feeders and transformers, with the incorporation of new BESS units while optimizing the size of the BESS. The algorithm also determines a plan life based on consideration of the life of various assets. The objective function minimizes the total annualized cost comprising the costs of new and replacement assets, the costs of operation and maintenance, and the costs associated with customer interruptions. The formulation is constrained by limits on bus 
voltages, feeder capacities, BESS unit capacities and the bus-power balance equations. The problem formulation and its constraints are given below:

\subsubsection{Objective Function}

The objective function (2.1) searches for the best size of the BESS and number of required feeders while minimizing the total costs of the annualized asset solution, as set out below:

$f_{S F}(\overline{S F}, N Y)+f_{B}(\overline{P B}, N Y)+f_{r e p B}(\overline{P B}, N Y)+f_{m c F}(N Y)+f_{m c B}(N Y)+f_{C I C}(S F E, \overline{S F}, N Y)$

The total cost in (2.1) comprises two main segments. Capital costs and risk costs, referring to Fig. 1.4

\subsubsection{Annualized Capital Costs}

The capital cost includes the followings: feeder costs (2.2); BESS unit costs (2.3); BESS unit replacement costs (2.4) and (2.5); feeder maintenance costs (2.6); BESS maintenance costs (2.7); and customer interruption costs (2.8) and (2.9). The detailed formulation for each is explained down below.

The annualized costs of new feeder sections are computed as follows:

$$
f_{S F}(\overline{S F}, N Y)=\frac{r(1+r)^{N Y}}{(1+r)^{N Y}-1} \cdot \sum_{l=1}^{N L} K F_{l} \cdot\left(N C_{l}\right) \cdot \overline{S F_{l}}
$$

where $K F_{l}$ is the feeder cost constant, $N C_{l}$ is the number of feeder circuits, and $\overline{S F_{l}}$ is the peak flow of the feeder. 
The term $\frac{r(1+r)^{N Y}}{(1+r)^{N Y}-1}$ annualizes the cost for the present year based on consideration of a plan life of NY years and an interest rate of $r$. (Detailed explanation can be found in Appendix A).

The annualized cost of the BESS units is calculated as follows:

$$
f_{B}(\overline{S B}, N Y)=\frac{r(1+r)^{N Y}}{(1+r)^{N Y}-1} \cdot \sum_{i=1}^{N X}\left(K B P \cdot \overline{P B_{l}}+K B E \cdot \overline{P B_{l}}\right)
$$

where $K B P$ and $K B E$ correspond to the cost constants for power and energy, respectively, of the BESS units, and $\overline{P B_{l}}$ and $\overline{E B_{l}}$ are the respective optimized power and energy capacities of the BESS unit at bus $i$.

In this formulation, the replacement of the BESS units due to degradation is based on the following justification (2.5). Given the current state of the technology, feeder life is usually several times that of BESS units. In the DSA planning formulation, $N Y$ is the total number of useful years and is determined optimally, with the longest-lasting asset assumed to be the feeders. It is therefore necessary to make use of several BESS units during the life of a feeder. $N R$ represents the number of replacements, and $B Y$ indicates the useful life time of one BESS unit:

$$
N R=N Y / B Y
$$

The annualized cost of replacing the BESS units is then given as:

$$
f_{\text {rep } B}(\overline{S B}, N Y)=\frac{r(1+r)^{N Y}}{(1+r)^{N Y}-1} \cdot \sum_{i=1}^{N X} \sum_{n=1}^{N R} \frac{\left(K B P R \cdot \overline{P B_{l}}+K B E R \cdot \overline{E B_{l}}\right)}{(1+r)^{n \cdot B Y}}
$$

where $K B P R$ and $K B E R$ correspond to the replacement cost constants for BESS unit power and energy, respectively. 


\subsubsection{Annualized Risk and Maintenance Cost}

The annualized feeder maintenance cost is as follows:

$$
f_{m c f}(N Y)=\frac{r(1+r)^{N Y}}{(1+r)^{N Y}-1} \cdot \sum_{n=1}^{N Y} \frac{K_{m f 0}\left(1+K_{m f}\right)^{n}}{(1+r)^{n}}
$$

where $K_{m f 0}$ is the present initial maintenance cost of the feeders, and $K_{m f}$ is the growth rate for maintenance costs.

Considering both maintenance and replacement, the annualized maintenance cost of the BESS units is calculated as follows:

$$
f_{m c B}(N Y)=\sum_{i=0}^{N R-1} \sum_{n=1}^{B Y-1} \frac{K_{B f}\left(1+K_{B v}\right)^{n}}{(1+r)^{n+i \cdot B Y}}+\sum_{n=1}^{\substack{N Y-\\ N R * B Y}} \frac{K_{B f}\left(1+K_{B v}\right)^{n}}{(1+r)^{n+N R \cdot B Y}}
$$

where $K_{B f}$ is the initial present maintenance cost of the BESS units, and $K_{B v}$ is the growth rate for maintenance costs. The first part of the equation deals with the maintenance cost of BESS and the second part of (3.7) calculated the maintenance cost of the BESS replacement.

\subsubsection{SAIDI and Customer Interruption Cost Calculation}

Planning from the customer's perspective, the customer's net annual cost must be minimized. This includes two components: (1) asset and energy costs, and, (2) CIC. The plan must minimize the total cost for customers. Hence, minimizing CIC concurrently with minimizing asset costs is very important.

Feeder interruptions and their resulting impact on reliability and costs to the customers are a function of feeder usage. If a feeder is always lightly loaded, then it provides the greatest reliability with the lowest customer interruption costs. Conversely, feeders that are heavily loaded at all times are associated with the lowest reliability levels and the highest customer interruption costs. This 
phenomenon can be investigated by computing the reliability at each loading level, and then multiplying the result by the probability of the feeder being at that loading level to obtain a weight for each result. This calculation is then repeated for each feeder, ranging from light peak loading to heavy peak loading. The final result indicates the relationship of the level of reliability, expressed in terms of the System Average Interruption Duration Index (SAIDI), to the ratio of the peak load divided by the feeder capacity. The customer interruption cost can then be estimated from the computation of the SAIDI value for a feeder section as a function of the peak load. The SAIDI per feeder circuit value can be computed as follows, based on [65]. (More details about the derivation of (2.8) and constant values of A, B and C can be found in Appendix A):

$$
\operatorname{SAIDI}(S F E, \overline{S F})=\frac{A\left(\frac{\max _{t}\left\{S F_{l t}\right\}}{N C_{l} \cdot S F E_{l}}\right)^{2}+B\left(\frac{\max _{t}\left\{S F_{l t}\right\}}{N C_{l} \cdot S F E_{l}}\right)+C}{N C_{l}}
$$

The annual Customer Interruption Cost (CIC) is computed as follows [25]:

$$
f_{C I C}(S F, N Y)=\frac{r(1+r)^{N Y}}{(1+r)^{N Y}-1} \cdot \sum_{n=1}^{N Y} \sum_{l=1}^{N L} \frac{\operatorname{SAIDI}(S F E, \overline{S F}) \cdot K r_{0}\left(1+K r_{r}\right)^{N Y}}{N C_{l} \cdot(1+r)^{n}}
$$

Eqs. (2.2) to (2.9) are the computations for the objective function expressed in (2.1). $N Y$ is a variable whose value is optimally determined as part of the solving of this formulation.

\subsubsection{Constraints and Boundaries}

The proposed DSA planning formulation minimizes the objective function (2.1) while satisfying the following constraints imposed by the network model and equipment limits. The equations that model the energy in the BESS units make the formulation inter-temporal and establish the resulting complexity of the solution space. 
Since the feeders are in parallel, the new upgraded line admittance is equal to the previous admittance multiplied by the number of upgraded feeders:

$$
y_{l l}=y_{l} \cdot N C_{l}
$$

The complex bus-wise power balance equation is computed for time $t$ as follows:

$$
S D_{i t}+S L_{i t}\left(V_{i t}, \delta_{i t}, y_{l l}\right)-S G_{i t}-S B_{i t}=0
$$

where $S G$ is the vector of the bus-wise apparent power generation, $S D$ is the vector of the bus-wise apparent power loads, and $S L(V, \delta)$ is the vector of the apparent power injected from the buses into the connected lines. $S B$ is the vector of the apparent power of the BESS units. (For more detailed derivation, refer to equations A.1 and A.2 in Appendix A).

The power flow in feeder segment $l$ between buses $i$ and $j$ at time $t$ is computed as follows:

$$
S F_{l}-V_{i t} \angle \delta_{i t} \cdot\left[\left(V_{i t} \angle \delta_{i t}-V_{j t} \angle \delta_{j t}\right) \cdot y_{l l} \angle \theta_{l}\right]^{*}=0
$$

The energy in BESS unit $i$ at time $t$ can be computed as follows:

$$
E B_{i t}=H T_{t} \cdot \max \left(P B_{i t} \cdot K_{P} \frac{P B_{i t}}{K_{P}}\right)+E B_{i, t-1} \cdot K_{E}
$$

where $K_{P}$ models the efficiency of the power conversion system, and $K_{E}$ accounts for any selfdischarge.

In addition, the sum of the energy stored and retrieved from a BESS unit is constrained to equal zero over the study period, with $P B$ being the vector of the active power of the BESS unit.

$$
\sum_{t=1}^{N H} H T_{t} \cdot P B_{i t}=0 ; i=1,2 \ldots N X
$$

The formulation is also subject to the following boundaries:

Limits on the bus voltage magnitudes:

$$
\underline{V} \leq V_{i t} \leq \bar{V}
$$


Limits on complex power generated by the sources:

$$
\underline{S G_{i}} \leq S G_{i t} \leq \overline{S G_{i}}
$$

BESS power limits:

$$
\underline{S B} \leq S B_{i t} \leq \overline{S B} \quad i=1,2 \ldots N X
$$

Complex line flow limits:

$$
\left|S F_{l t}\right| \leq \overline{S F_{l}}
$$

Solving the optimization formulation (2.1), detailed in (2.10) to (2.18) above, enables the minimization of the annualized DSA investment cost and the determination of the optimal number of years for the best asset life.

\subsection{Solution Algorithm}

As previously mentioned, the proposed DSA planning formulation and algorithm includes planning for traditional assets, such as feeders and transformers, as well as for the addition of new BESS units while optimally sizing BESS units. It is important to clarify that the proposed model as previously presented in (2.1), (2.10) to (2.18) is a Mixed Integer Nonlinear problem (MINLP). This optimization method was implemented in the MATLAB programming language using OPTI Toolbox and an interior-point algorithm. 


\subsection{Test Results and Discussions}

In this section, the proposed method is applied in two different systems. A 3-bus simplified Canadian system and IEEE 33-bus system. Also, the economic data is presented. The details are given in the following sections.

\subsubsection{Economic Data}

The systems were analyzed in the context of undergrounded urban systems with critical infrastructure for which customer interruption costs are significant. Based on the Final Report by InfraSource Technology [66], the assumed costs are as shown in Table 2.1.

The real-estate cost for both BESS and feeders is not considered in the algorithm, but in cases that the price of real-estate is considered crucial in the planning process, it can be added to the algorithm as a constant in equations (2.2) and (2.3). 
Table 2.1 Feeder and BESS Costs (US\$)

\begin{tabular}{l|l}
\hline \multicolumn{1}{c|}{ Item } & Value \\
\hline$K F-$ Underground Feeder cost coefficient (\$/MVA) & US $\$ 1,350,000$ \\
\hline$K B E-$ Energy cost coefficient for a lithium-ion battery BESS unit (\$/MWh) & US\$100,000 \\
\hline$K B P$ - Power cost coefficient for a lithium-ion battery BESS unit (\$/MW) & US $\$ 300,000$ \\
\hline Customer interruption cost (\$/minute) & US $\$ 40,000$ \\
\hline Underground feeder maintenance cost (\$/year) & US $\$ 9,000$ \\
\hline BESS maintenance cost (\$/year) & US $\$ 1,000$ \\
\hline BESS replacement period (years) & 10 \\
\hline Replacement Cost for Battery Power Capacity (\$/MW) & US $\$ 210,000$ \\
\hline Replacement Cost for Battery Energy Capacity per (\$/MWh) & US $\$ 70,000$ \\
\hline Interest rate (per year) & $5 \%$ \\
\hline Power Conversion Efficiency of BESS & $88 \%$ \\
\hline
\end{tabular}

\subsubsection{Simplified Canadian Distribution System Data (3-bus)}

A simplified Canadian distribution system with three buses as depicted in Fig. 2.1 is considered for this work. The voltage at feeder source is set as 1.03 per unit, based on the nominal voltage of $27.6 \mathrm{kV}$. The equivalent impedances and loads are listed in Table 2.2 and Table 2.3. 


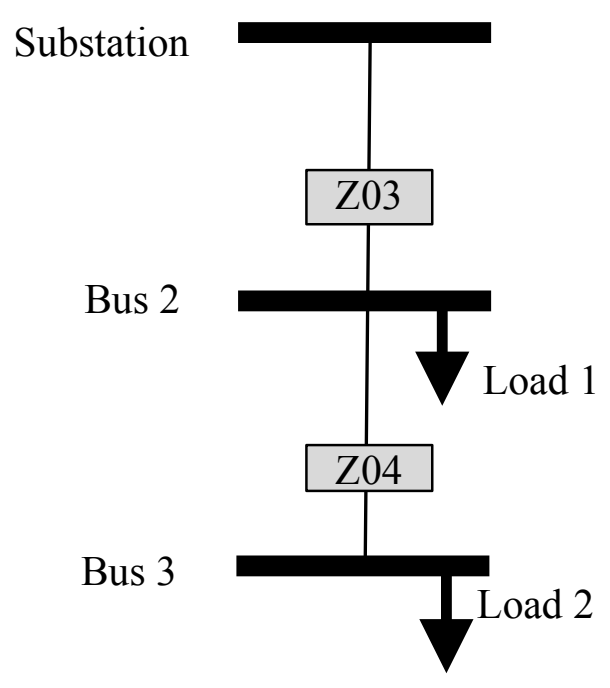

Fig. 2.1 3-bus Network

Table 2.2 Impedances in the Network in $27.6 \mathrm{kV}$

\begin{tabular}{c|c|c|c}
\hline & $\mathrm{R}(\Omega)$ & $\mathrm{X}(\Omega)$ & $\begin{array}{c}\text { Feeder Planning } \\
\text { Load Limit (MVA) }\end{array}$ \\
\hline $\mathrm{Z03}$ & 2.3 & 6.2 & 20 \\
\hline $\mathrm{Z04}$ & 0.4 & 0.9 & 20 \\
\hline
\end{tabular}

Table 2.3 Loads in the Network in $27.6 \mathrm{kV}$

\begin{tabular}{c|c|c}
\hline & PD (MW) & Power Factor \\
\hline Load \#1 & 12 & $95 \%$ \\
\hline Load \#2 & 7 & $95 \%$ \\
\hline
\end{tabular}


The base values used in this work are listed in Table 2.4.

Table 2.4 Base Values for 27.6kV System

\begin{tabular}{c|c}
\hline Voltage base & $27.6 \mathrm{kV}$ \\
\hline Power base & $10 \mathrm{MVA}$ \\
\hline Impedance base & $76.1 \Omega$ \\
\hline
\end{tabular}

By applying these base values, per unit values of voltage, power and impedances are calculated.

The load flow is solved using Newton-Raphson technique and the results obtained are recorded in Table 2.5.

Table 2.5 Voltage Solutions from Load Flow

\begin{tabular}{l|l}
\hline Buses & V (per unit) \\
\hline Bus \#1 & $1.03<0.00^{\circ}$ \\
\hline Bus \#2 & $1.02<-0.01^{\circ}$ \\
\hline Bus \#3 & $1.01<-0.01^{\circ}$ \\
\hline
\end{tabular}

The active and reactive powers supplied from feeder source are 19.13 MW and 5.9 MVAr respectively. The line flow through the circuit is given in Table 2.6. 
Table 2.6 Line Flow of the 3-bus System

\begin{tabular}{c|c|c}
\hline & Line flow per circuit (MVA) & Feeder Planning Load Limit (MVA) \\
\hline Section \#1 & 19.9 & 20 \\
\hline Section \#2 & 7.2 & 20 \\
\hline
\end{tabular}

The network integrated with energy storage device is shown in Fig. 2.2. In this work, Lithium-ion batteries are used as the energy storage device.

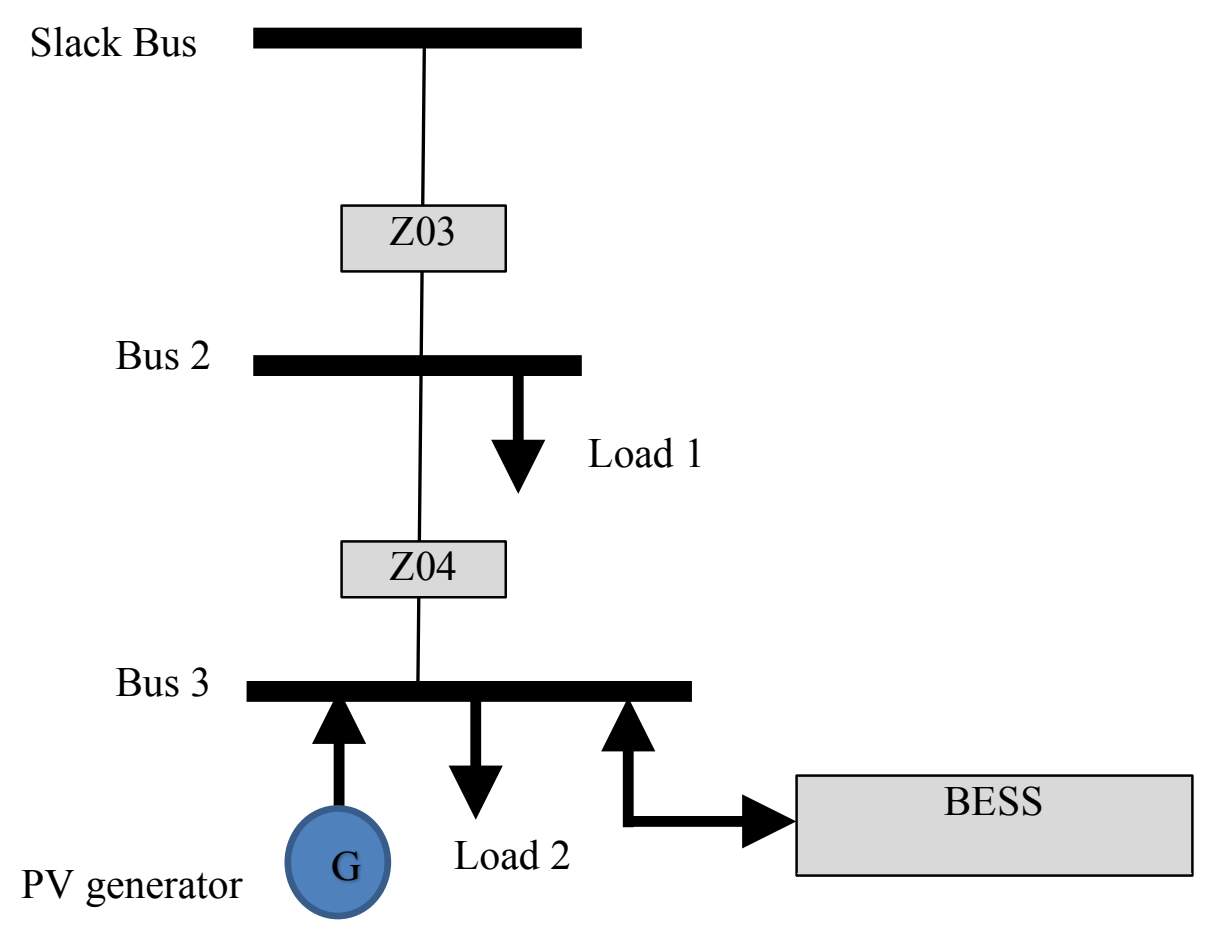

Fig. 2.2 System with BESS and PV

A PV generator with $30 \mathrm{MW}$ of rated power is connected to Bus \#3. To check the method performance, the load and generation scenarios were divided in three zones as follows:

a. $25 \%$ peak load (as a light load) and $90 \%$ PV generation - Time Zone 1 (5 hours) 
b. $60 \%$ peak load and $60 \%$ PV generation - Time Zone 2 (6 hours)

c. $100 \%$ peak load and $0 \% \mathrm{PV}$ generation - Time Zone 3 ( 13 hours)

The load and generation values for each region are provided in Table 2.7 and they present the worst case scenario of one day (24 hours), where PD stands for Power Demand and PV for Photovoltaic generation.

Table 2.7 Power Demand (PD) and PV Generation (PV) Values for Each Time Zone

\begin{tabular}{c|c|c|c}
\hline & Time Zone 1 & Time Zone 2 & Time Zone 3 \\
& 5 hours & 6 hours & 13 hours \\
\hline PD (MW) & 4.7 & 11.4 & 19 \\
& $(25 \%$ peak load $)$ & $(60 \%$ peak load $)$ & $(100 \%$ peak load $)$ \\
\hline PV (MW) & $27(90 \%$ PV $)$ & $18(60 \%$ PV $)$ & $0(0 \%$ PV $)$ \\
\hline
\end{tabular}

The power flow problem is solved, and the results for the varied load profiles and PV generation are listed in Table 2.8 and Table 2.9. 
Table 2.8 Voltage Solutions for Each Time Zone

\begin{tabular}{c|c|c|c}
\hline \multirow{2}{*}{} & \multicolumn{3}{|c}{ Voltage Solutions (per unit) } \\
\hline & Time Zone 1 & Time Zone 2 & Time Zone 3 \\
& 5 hours & 6 hours & 13 hours \\
\hline Bus \#1 & $1.03 \angle 0.00^{\circ}$ & $1.03 \angle 0.000^{\circ}$ & $1.03 \angle 0.000^{\circ}$ \\
\hline Bus \#2 & $1.03 \angle 0.070^{\circ}$ & $1.02 \angle 0.020^{\circ}$ & $0.99 \angle-0.060^{\circ}$ \\
\hline Bus \#3 & $1.03 \angle 0.150^{\circ}$ & $1.02 \angle 0.070^{\circ}$ & $0.98 \angle-0.080^{\circ}$ \\
\hline
\end{tabular}

Table 2.9 Line Flows for Each Time Zone

\begin{tabular}{c|c|c|c|c}
\hline & \multicolumn{2}{|c|}{ Line Power Flows Per Circuit (MVA) } & Feeder \\
\hline & Time zone 1 & Time zone 2 & Time zone 3 & Capacity \\
& 5 hours & 6 hours & 13 hours & (MVA) \\
\hline Z01 & $22.3^{*}$ & 7.5 & 19.9 & 20 \\
\hline Z02 & $25.2^{*}$ & 13.8 & 7.3 & 20 \\
\hline
\end{tabular}

Note: $(*)$ Overload

It should be noted that feeder sections 1 and 2, during Time Zone 1, are overloaded above the 20 MVA rated capacity. From the power flow results given in Table 2.9, an appreciable increase in the line flow above the feeder capacity can be observed in feeder sections Z01 and Z02 as the load profile and PV generation vary. The feeder section therefore requires upgrades. The PD and PV variations and line flows at different time zones are plotted in Fig. 2.3. 


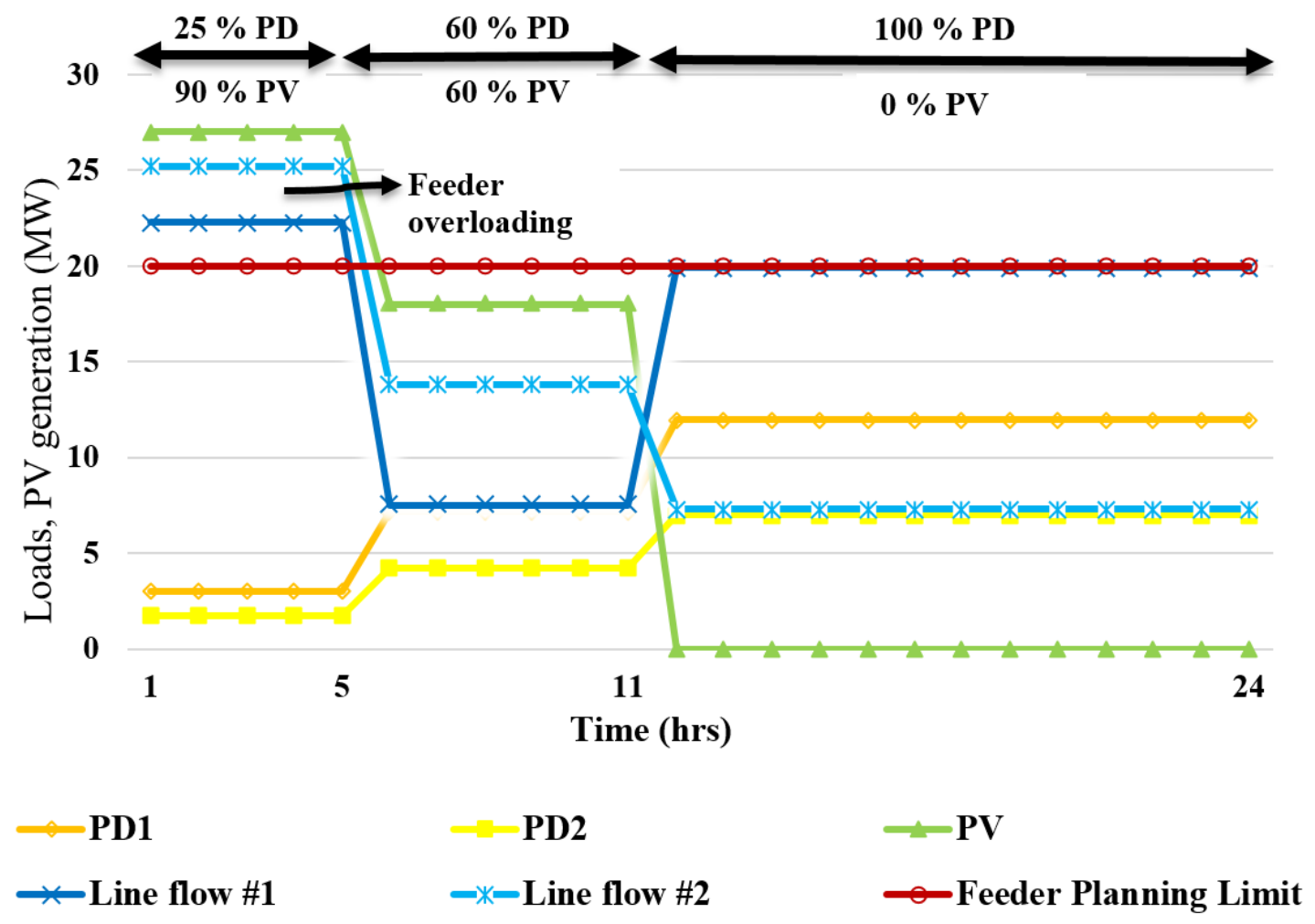

Fig. 2.3 Time vs. Load and Generation in Section \#1 and Section \#2

\subsubsection{Optimal Planning of a Canadian Distribution System}

For the work presented in this thesis, a lithium-ion battery BESS unit was used. The proposed DSA optimal planning formulation with BESS units was solved for the cases listed below. For each case, the proposed method establishes the optimal plan that results in the lowest total amortized annual cost solution. The data used for this optimization problem are provided in Table 2.1 to Table 2.3 .

The two cases for which the optimization problem is solved are as follows:

- Case I: Conventional DSA planning model that considers only feeders (i.e., no BESS units)

- Case II:Proposed DSA planning model that considers feeders and BESS units 
The line power flow results and the maximum number of feeders required for each case are listed in Table 2.10.

Table 2.10 Total Line Power Flow in the Feeders for the 3-Bus System Cases

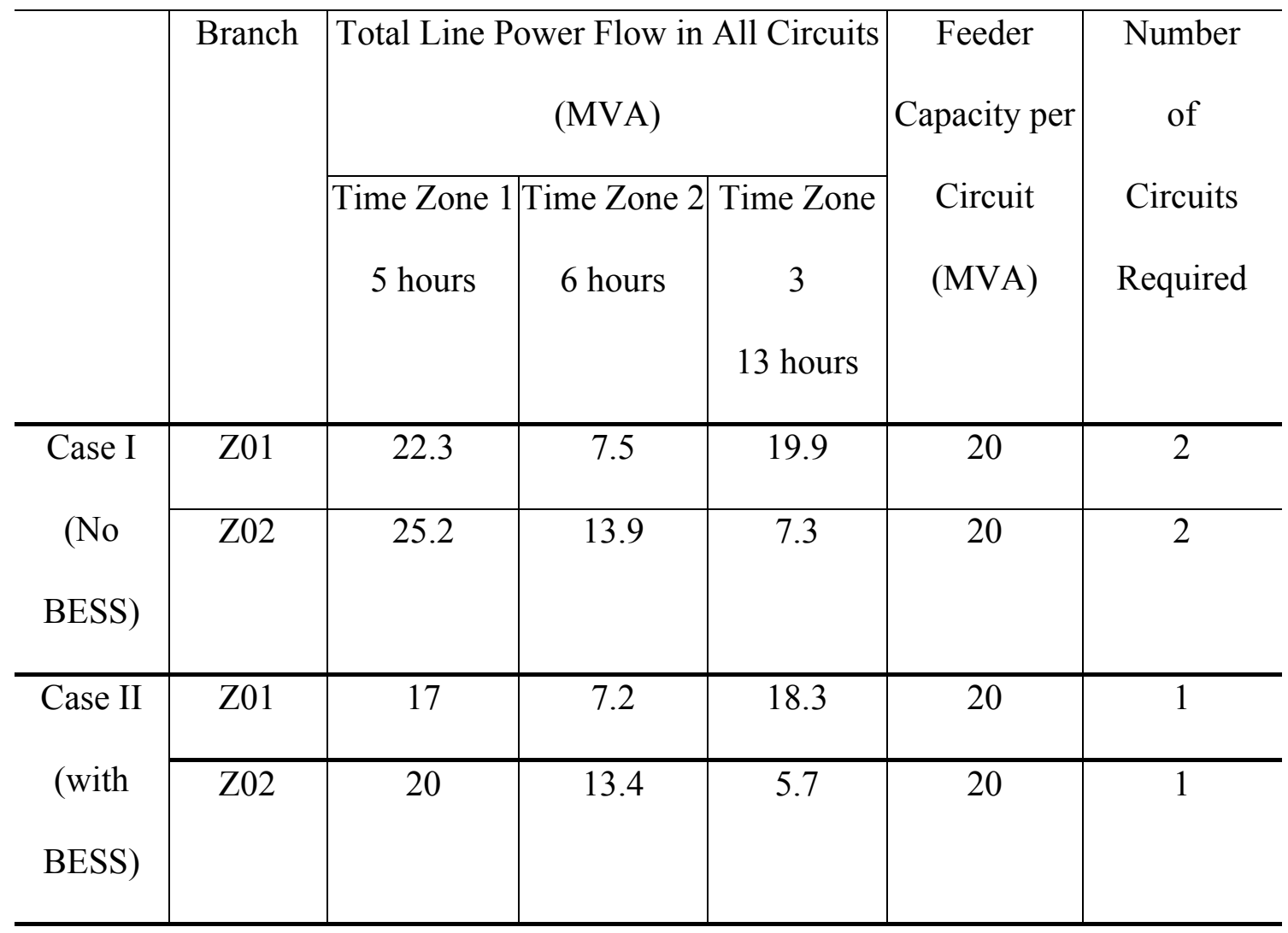

In case I, with no consideration of BESS units, during Time Zone 1, the line flow exceeds the feeder planning limit in the first feeder section. Therefore, an additional feeder is required in that section. The real and reactive power supplied from feeder source are listed in Table 2.11. The directions of flows can be seen in Fig. 2.4. 
Table 2.11 Power Supplied from Feeder Source - Case I

\begin{tabular}{l|c|c|c}
\hline & Time zone 1 & Time zone 2 & Time zone 3 \\
\hline PG (MW) & -22.2 & -6.6 & 19.0 \\
\hline QG (MVAr) & 1.6 & 3.6 & 6.0 \\
\hline
\end{tabular}

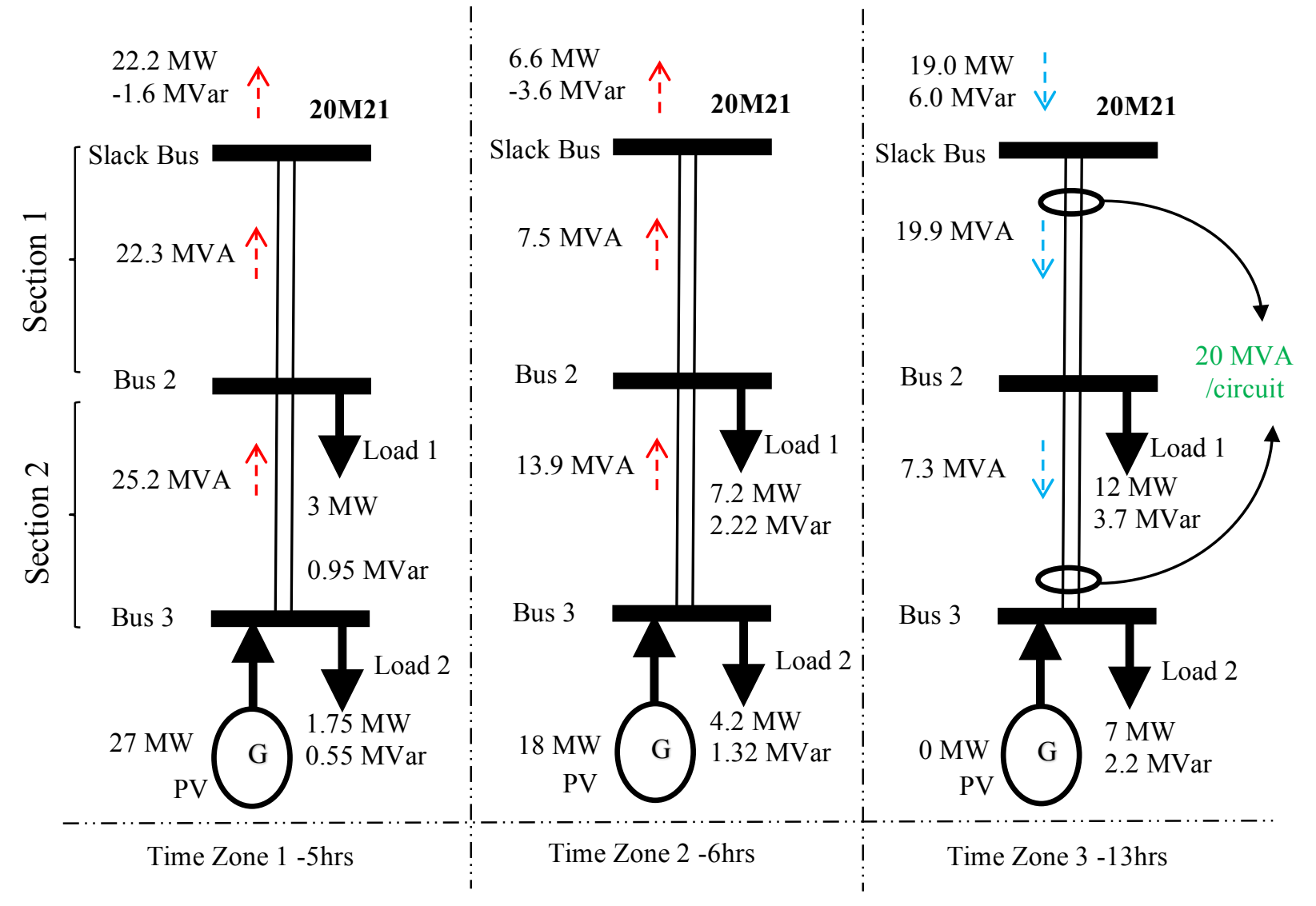

Fig. 2.4 Power Flows in Feeders with No Battery ES - Case I 
From a financial perspective, the estimated optimal number of years is 45 . The total amortized annual cost for creating and operating this solution is US\$ 3,512,855/year, which is composed of the following costs:

Annualized Feeder Upgrade Costs: $\quad$ US\$ 3,043,553

Annualized Customer Interruption Costs: US\$ 446,983

Annualized Feeder Maintenance Costs: $\quad$ US\$22,319

Total annualized Costs: $\quad$ US\$ 3,512,855

In case II, with the existence of BESS units, the line flow is within the feeder planning limit during all the time zones for both sections, so that additional feeders are not required. For case II, the power supplied from the feeder source is shown in Table 2.12, and the optimal BESS unit energy and power for each time zone to meet load requirements and to maintain the line power flow within the feeder capacity are as listed in Table 2.13. The negative sign in Table 2.13 means that the BESS is charging. The BESS unit power and energy capacities planned for case II are 5.22 MW and 22.98 MWh, respectively.

Table 2.12 Power Supplied from Feeder Source - Case II

\begin{tabular}{l|c|c|c}
\hline & Time zone 1 & Time zone 2 & Time zone 3 \\
\hline PG (MW) & 17.0 & 6.1 & 17.6 \\
\hline QG (MVAR) & 1.4 & 3.7 & 5.0 \\
\hline
\end{tabular}

Table 2.13 BESS Power and Energy for Case II

\begin{tabular}{c|c|c|c}
\hline & Time Zone 1 & Time Zone 2 & Time Zone 3 \\
\hline BESS Power (MW) & -5.22 & -0.44 & 1.40 \\
\hline BESS Energy $(\mathrm{MWh})$ & 22.98 & 22.98 & 0 \\
\hline
\end{tabular}


For Case II, the directions of flows can be seen in Fig. 2.5 with the presence of BESS.

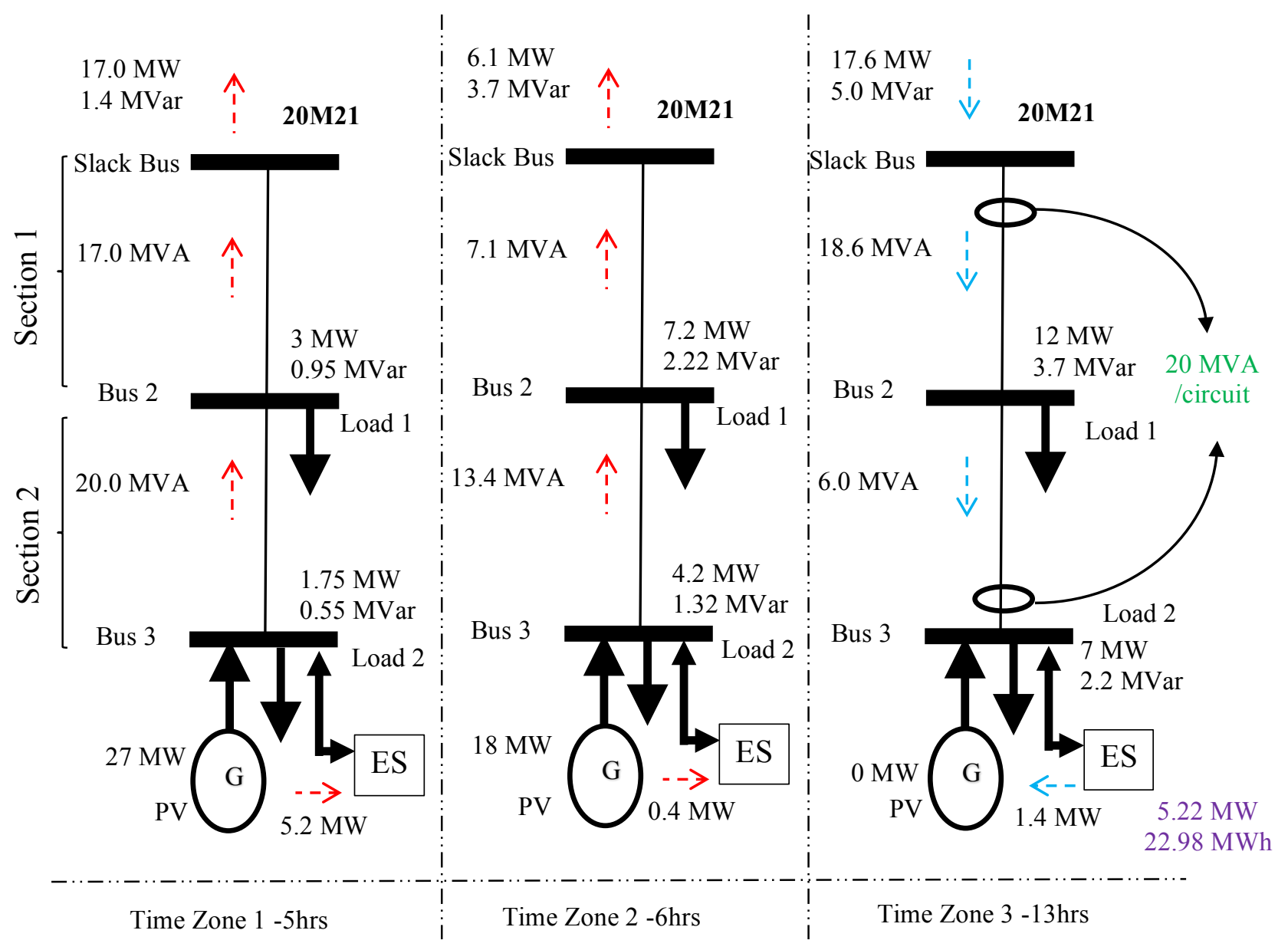

Fig. 2.5 Power Flows in Feeders with Battery ES - Case II

Power efficiency and battery energy efficiency for each time zone are shown in Fig. 2.6, assuming the efficiency is $88 \%$. 

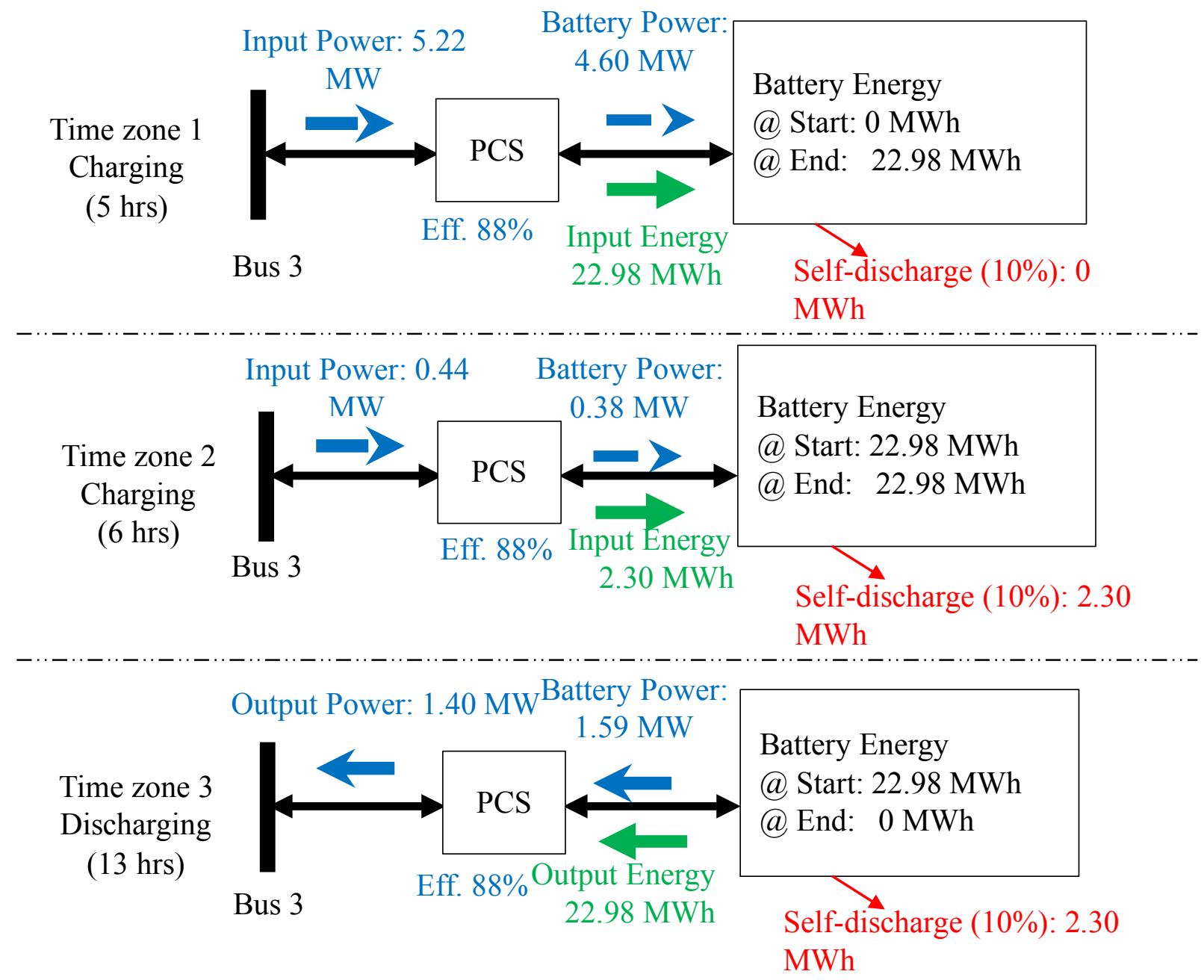

Fig. 2.6 Battery ES Energy and Power Efficiency - Case II 
The estimated financially optimal number of years is 46 . The total amortized annual cost for creating and operating this solution comes to US\$ 894,718/year, which is composed of the following costs:

Annualized Feeder Upgrade Costs: $\quad$ US\$ 0

Annualized Customer Interruption Costs: $\quad$ US\$ 400,544

Annualized Feeder Maintenance Costs: $\quad$ US\$ 15,001

Annualized BESS Costs: $\quad$ US\$280,132

Annualized Battery Replacement Costs: $\quad$ US\$ 194,289

Annualized Battery Maintenance Costs: $\quad$ US\$ 4,752

Total annualized Costs: $\quad$ US $\$ 894,718$

It can be concluded, for this specific case, that the cost associated with planning to connect BESS units is lower than the cost in the other case, in which the addition of two feeders is required. Hence, for the 3-bus Canadian system presented, using the DSA planning tool and minimizing the equivalent annual costs avoids the requirement to upgrade the feeders.

\subsubsection{Sensitivity Analysis for the Canadian Distribution System}

This section presents a sensitivity analysis of the proposed method with respect to costs and other critical aspects. In general, the load profile may vary on a daily basis, which affects the results. The average demand at the substation for one day considering the load and generation is indicated as $\mathrm{P}_{\text {avg. }}$ 
The peak demand at the substation for one day is denoted as $\mathrm{P}_{\max }$. The $\mathrm{P}_{\text {avg }}$ and $\mathrm{P}_{\max }$ values are computed as follows:

$$
\begin{gathered}
P_{\max }=\max _{t=1: N H}\left\{\operatorname{real}\left\{\sum_{i=1}^{N B}\left(S D_{i t}-S G_{i t}\right)\right\}\right\} \\
P_{\text {avg }}=\operatorname{real}\left\{\frac{1}{\sum_{t=1}^{N H} H T_{t}} \sum_{t=1}^{N H} \sum_{i=1}^{N B}\left(S D_{i t}-S G_{i t}\right)\right\}
\end{gathered}
$$

For the purposes of analysis, the loading of the 3-bus Canadian system was altered by varying the $\mathrm{P}_{\mathrm{avg}} / \mathrm{P}_{\max }$ ratio, which significantly influences the potential for BESS units to be chosen as assets.

The DSA planning model was solved considering an aggregated 24-hour period divided into three time zones. The sensitivity analysis involved three scenarios, as explained below. It is important to highlight that none of the scenarios include PV.

\section{1) Study Condition: $\mathrm{P}_{\mathrm{avg}}$ Kept Constant, $\mathrm{P}_{\max } \underline{\text { Increased: }}$}

In this case, the value of $\mathrm{P}_{\mathrm{avg}}$ is kept constant at $15.8 \mathrm{MW}$, and the value of $\mathrm{P}_{\max }$ is gradually increased from $19 \mathrm{MW}$ to $28.5 \mathrm{MW}$, so that the $\mathrm{P}_{\mathrm{avg}} / \mathrm{P}_{\max }$ ratio decreases from 0.83 to 0.55 . The results are presented in Fig. 2.7 showing the annual prices for the planning period. It can be seen in the figure that when $\mathrm{P}_{\mathrm{avg}}$ is constant, the opportunity for the effective use of BESS increases with an increase in $\mathrm{P}_{\max }$. For this case, the BESS varies from 10.2 MVA to 0 MVA and there is a higher arbitrage opportunity for BESS when the $\mathrm{P}_{\text {avg }} / \mathrm{P}_{\max }$ ratio decreases. Table 2.14 shows the load profile at each time zone for points 0.55 and 0.83 . 


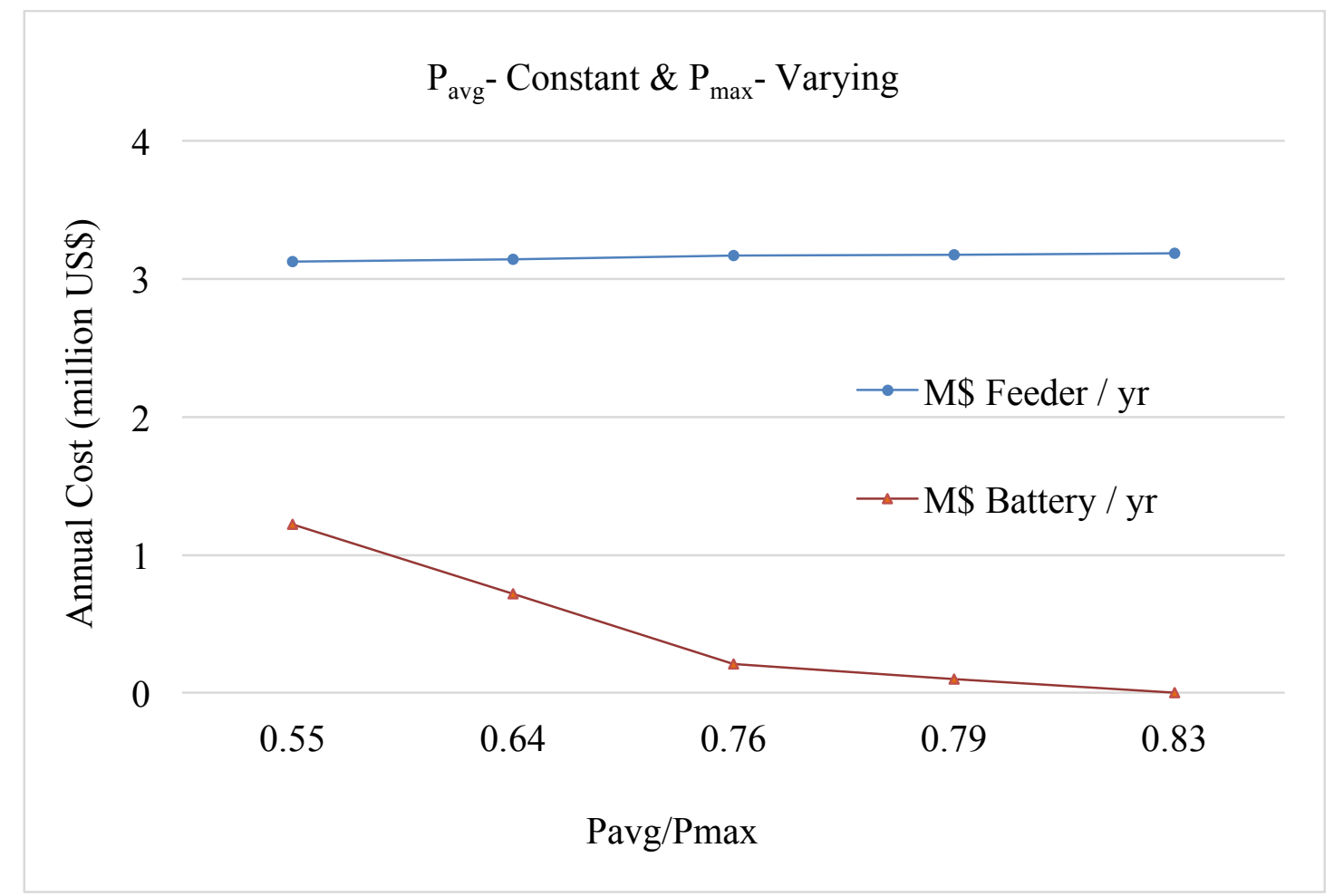

Fig. 2.7 Loading Factor versus Costs of Feeders and BESS for Constant $P_{\text {avg }}$ Value and Varied

$$
\mathrm{P}_{\max } \text { Value. }
$$

Table 2.14 Power Demand (PD) and Power Supplied by the Substation (PG) in MW

\begin{tabular}{c|c|c|c|c}
\hline \multirow{2}{*}{ Pavg/Pmax } & & $\begin{array}{c}\text { Time Zone 1 } \\
5 \text { hours }\end{array}$ & $\begin{array}{c}\text { Time Zone 2 } \\
6 \text { hours }\end{array}$ & $\begin{array}{c}\text { Time Zone 3 } \\
13 \text { hours }\end{array}$ \\
\hline \multirow{2}{*}{0.55} & PD & $9.5(50 \%)$ & $28.5(150 \%)$ & $12.35(65 \%)$ \\
\cline { 2 - 5 } & PG & 15.5 & 18.3 & 17.3 \\
\hline \multirow{2}{*}{0.83} & PD & $17.1(90 \%)$ & $19(100 \%)$ & $13.9(73 \%)$ \\
\cline { 2 - 5 } & PG & 17.1 & 19 & 13.9 \\
\hline
\end{tabular}




\section{2) Study Condition: $\mathrm{P}_{\mathrm{avg}}$ Varied, $\mathrm{P}_{\max }$ Kept Constant:}

In this sensitivity analysis, the value of $\mathrm{P}_{\text {avg }}$ is increased from $22.8 \mathrm{MW}$ to $39.9 \mathrm{MW}$, while the value of $\mathrm{P}_{\max }$ is kept constant at $45.6 \mathrm{MW}$, which causes the $\mathrm{P}_{\mathrm{avg}} / \mathrm{P}_{\max }$ ratio to vary from 0.5 to 0.88 . The results are presented in Fig. 2.8. With increases in the value of $\mathrm{P}_{\text {avg }}$, the optimal BESS size varies in the range of $8.06 \mathrm{MVA}$ to $0 \mathrm{MVA}$, at the same time the optimal feeder size varies from 40 MVA ( 2 feeders) to 60 MVA ( 3 feeders) for section \#1, and remains constant at 20 MVA (1 feeder) for section \#2. The increase in the value of $\mathrm{P}_{\text {avg }}$ demands a larger feeder capacity and a smaller BESS capacity. As long as the $\mathrm{P}_{\text {avg }}$ value is within the feeder capacity of a circuit, the feeder cost is constant. When the $\mathrm{P}_{\text {avg }}$ value exceeds the feeder capacity of one circuit, another circuit is added, resulting in a discrete increase in feeder costs.

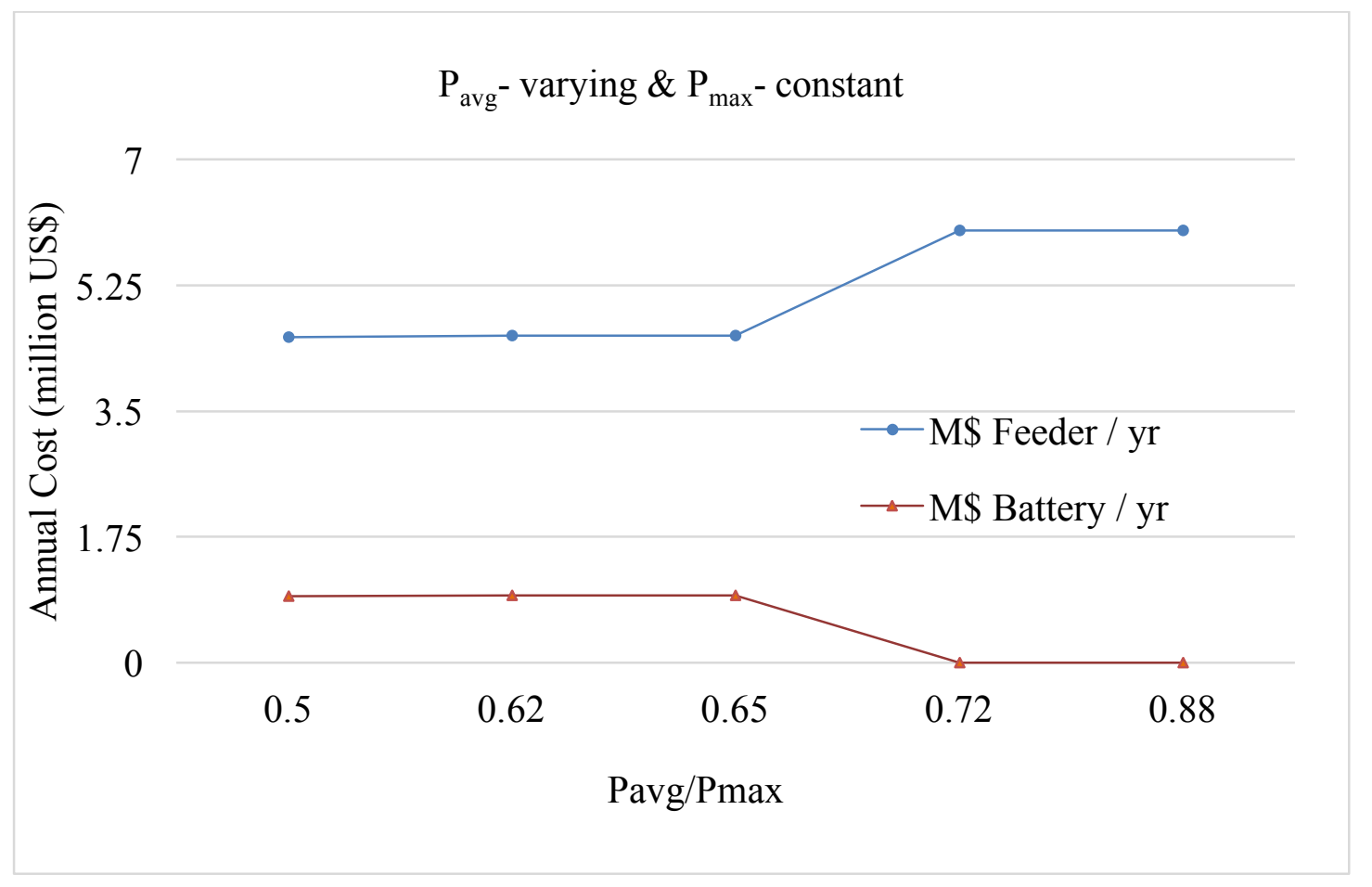

Fig. 2.8 Loading Factor versus Costs of the Feeders and BESS for a Constant $P_{\max }$ Value and Varied $\mathrm{P}_{\text {avg }}$ Value. 


\section{3) Sensitivity Analysis with the BESS Costs:}

To explore further the proposed DSA planning method, the BESS unit cost constants KBP (\$/MW) and $\mathrm{KBE}(\$ / \mathrm{MWh})$ were varied and added together, and the corresponding changes in the system costs were determined. As referenced from the Sandia Electricity Storage Handbook [67], the maximum KBP and KBE values were chosen as US\$ 800,000/MW and US\$ 800,000/MWh. Because it is expected in the future that the cost constants of BESS decrease, the KBP and KBE values are varied in this analysis, and an optimal plan is developed for each cost point using the proposed DSA planning algorithm. The total annual costs of the feeders and BESS units were obtained, which are shown in Fig. 2.9. Battery price prediction for future can be seen in Fig. 1.6.

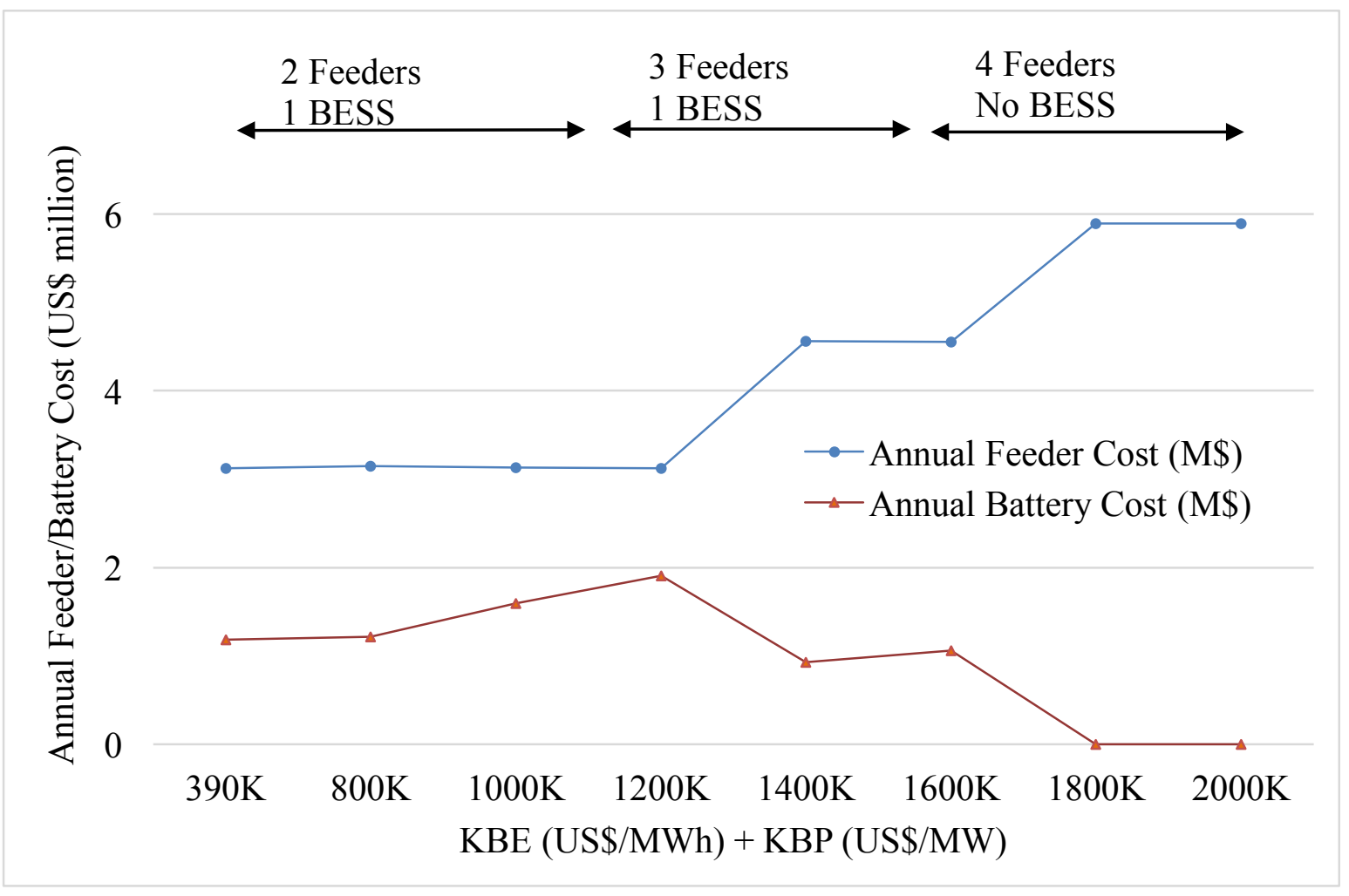

Fig. 2.9 Cost of Utilizing BESS and Feeders versus the BESS Cost Coefficient 
As one can see in Fig. 2.9, starting from the right side of the figure, the BESS unit is not utilized for values of KBP and KBE higher than US\$ 1,800,000, because in this case the usage of extra feeders is more economical. For lower values of KBP and KBE, starting from US\$1,200,000 up to US\$ $1,800,000$, the BESS unit is utilized, which is reflected in the reduced feeder cost. The same effect can be seen for values of KBE and KBP less than US\$1,200,000. In the figure, since the number of circuits in a feeder section changes as an integer in the model, the graph shows step changes.

\section{4) Sensitivity Analysis with the Feeder Cost:}

In all the analysis prepared so far, it has been assumed that the feeders were underground and therefore the feeder prices were assumed high. In this analysis, to show the capability of the proposed DSA planning method, the feeder cost constant KF (\$/MVA) is lowered, and the corresponding changes in the system costs is shown in Fig. 2.10. When the cost of utilizing feeders become less than US\$ 1,000,000 per MVA the proposed algorithm will not choose the BESS as an option and it will only increase the number of feeders. 


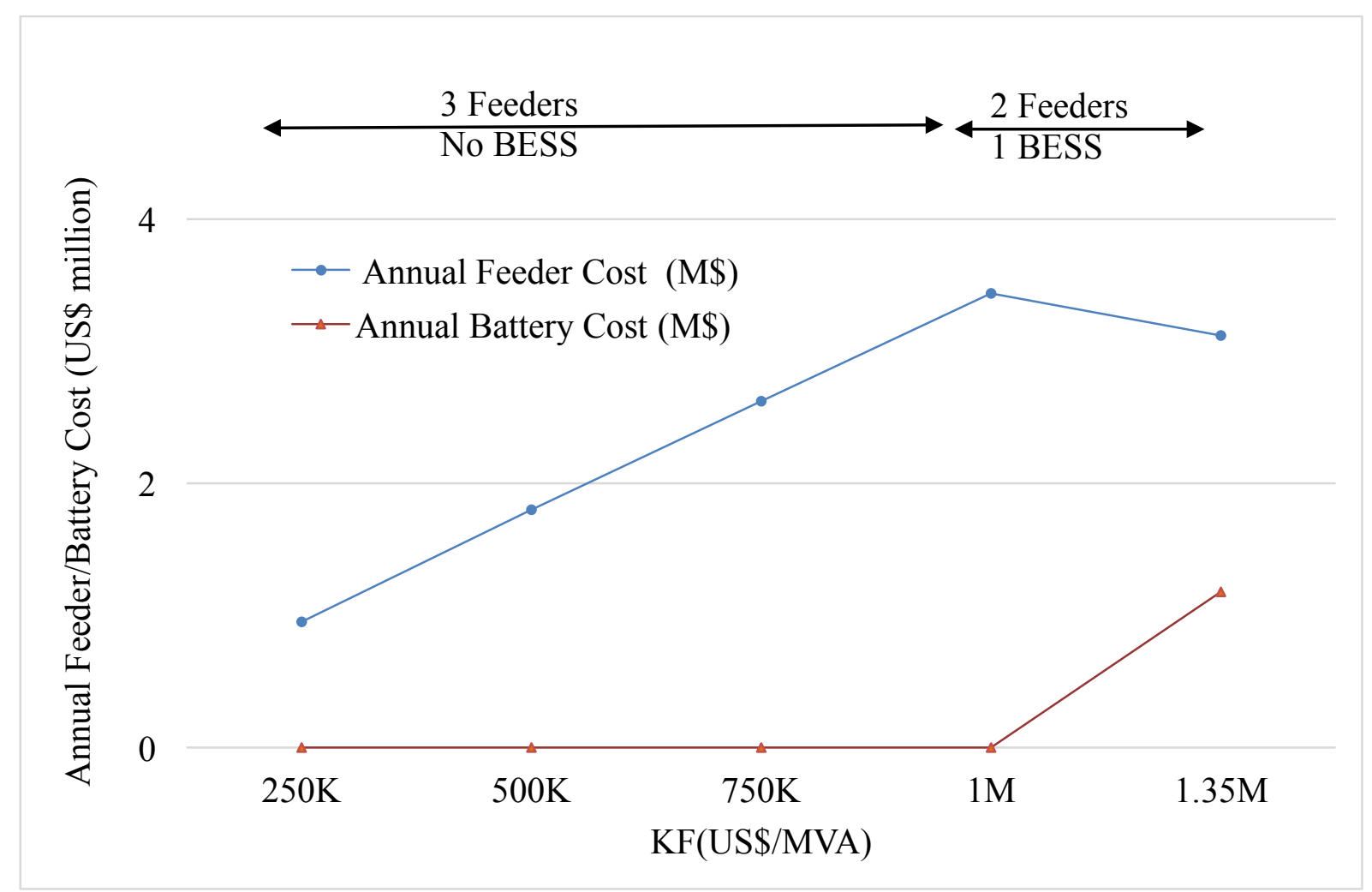

Fig. 2.10 Cost of Utilizing BESS and Feeders versus the Feeder Cost Coefficient

\subsubsection{Optimal Planning for the IEEE 33-bus Distribution System}

The proposed algorithm was also tested on the IEEE 33-bus system (Appendix B). The feeder capacity for the IEEE 33-bus system is assumed to be 5 MVA. A 7 MVA PV generator and one BESS unit are connected at bus \#24. The load and PV power variations for each time zone are listed in Table 2.15. 
Table 2.15 Power Demand (PD) and PV Generation (PV) for Each Time Zone in \%.

\begin{tabular}{c|c|c|c|c}
\hline & \multirow{2}{*}{ Bus \# } & Time Zone 1 & Time Zone 2 & Time Zone 3 \\
& & 8 hours & 8 hours & 8 hours \\
\hline PD & $2-33$ & $20 \%$ peak load & $50 \%$ peak load & $100 \%$ peak load \\
\hline PV & 24 & $100 \%$ PV & $50 \%$ PV & $0 \%$ PV \\
\hline
\end{tabular}

The optimization results for the IEEE 33-bus system are shown in Table 2.16, Table 2.17, and Table 2.18, which assist with comparison of two cases:

- Case I: with feeders only and no BESS units

-Case II: with both feeders and BESS units.

The following observations are based on the results:

In case I (no BESS), the loading on the feeders is higher, and the lowest estimated life cycle cost is US\$ $37,439,588$ which is composed of the following costs:

Annualized Feeder Costs:

US\$ 30,052,000

Annualized Customer Interruption Costs: $\quad$ US\$ 7,368,200

Annualized Feeder Maintenance Costs: $\quad$ US\$ 19,388

Total annualized Costs: $\quad$ US\$ $37,439,588$

The results of this case with no BESS are documented in Table 2.16 and indicate that two additional circuit feeders are needed to eliminate the overload of Sections \#1 and \#2 during Time Zone 2. 
Table 2.16 Total Line Power Flow for the 33-Bus System - Case I

\begin{tabular}{c|c|l|l|c|c}
\hline \multirow{2}{*}{ Section \# } & \multicolumn{3}{|c|}{ Line Power Flow (MVA) } & \multirow{2}{*}{ Feeder } & Maximum \# \\
\cline { 2 - 4 } & Time & Time & Time & Capacity & of Feeders \\
& Zone 1 & Zone 2 & Zone 3 & (MVA) & Required \\
\hline 1 & 1.7523 & 6.1742 & 3.9558 & 5 & 2 \\
\hline 2 & 1.5495 & 5.4628 & 3.4990 & 5 & 2 \\
\hline $3-32$ & $<5$ & $<5$ & $<5$ & 5 & 1 \\
\hline
\end{tabular}

In case II (with BESS), an optimal plan is developed whereby no additional feeders are necessary and only one BESS unit is sized, as one can see in Table 2.17. The minimum cost of the annual asset plan is calculated to be US\$ $36,509,475$ which is composed of the following costs:

Annualized Feeder Upgrade Costs: $\quad$ US\$ 29,000,000

Annualized Customer Interruption Costs: US\$ 7,402,000

Annualized Feeder Maintenance Costs: $\quad$ US\$ 19,037

Annualized BESS Costs: $\quad$ US\$ 47,011

Annualized Battery Replacement Costs: US\$ 40,219

Annualized Battery Maintenance Costs: US\$ 1,208

Total annualized Costs: $\quad$ US\$ $36,509,475$

Table 2.18 shows the energy and power in the BESS unit when it is optimally sized for 0.6744 MW and 3.9562 MWh. 
Table 2.17 Total Line Power Flow for the 33 Bus System - Case II

\begin{tabular}{|c|c|c|c|c|c|}
\hline \multirow[b]{2}{*}{ Section \# } & \multicolumn{3}{|c|}{ Line Power Flow (MVA) } & \multirow{2}{*}{$\begin{array}{l}\text { Feeder } \\
\text { Capacity } \\
\text { (MVA) }\end{array}$} & \multirow{2}{*}{$\begin{array}{c}\text { Maximum \# of } \\
\text { Feeders } \\
\text { Required }\end{array}$} \\
\hline & $\begin{array}{l}\text { Time } \\
\text { Zone } 1\end{array}$ & $\begin{array}{l}\text { Time } \\
\text { Zone } 2\end{array}$ & $\begin{array}{l}\text { Time } \\
\text { Zone } 3\end{array}$ & & \\
\hline 1 & 3.4785 & 3.7586 & 3.7091 & 5 & 1 \\
\hline 2 & 3.0696 & 3.2494 & 3.2494 & 5 & 1 \\
\hline $3-32$ & $<5$ & $<5$ & $<5$ & 5 & 1 \\
\hline
\end{tabular}

Table 2.18 Battery Power and Energy for Case II - 33-Bus System

\begin{tabular}{c|c|c|c}
\hline & Time Zone 1 & Time Zone 2 & Time Zone 3 \\
\hline Battery Power (MW) & -0.5034 & 0.6744 & -0.0824 \\
\hline Battery Energy (MWh) & 3.9562 & 0 & 1.2178 \\
\hline
\end{tabular}

From these results, it is clear that the proposed DSA planning method is able to include BESS units and additional feeder circuits into the problem solution. The proposed method considers a myriad of options to select the least costly annual asset plan. It compares the options evenly based on an evaluation of the total annual asset costs for delivering energy to the connected customers. 


\subsection{Chapter Summary}

As more intermittent generation, such as PV generation, and uncertain loads, such as Electric Vehicles, are integrated into expensive urban distribution systems, new solutions must be included into the Distribution System Asset (DSA) planning process. Battery Energy Storage Systems (BESS) have the potential to technically facilitate the integration of these technologies. In addition, as the cost of BESS is expected to decrease in the following years, this can also be an economic solution, especially in urban centers, where the installation of new feeders may be quite costly. Therefore, in this thesis a new model for the DSA planning process was proposed, which includes the possibility of installing BESS as a solution to integrate new loads and generators.

Based on the analyses presented here, one can see that the proposed model is flexible, automatically selecting the installation of new feeders and/or BESS while optimally sizing the BESS. In addition, the results show that in the presence of high penetration of PV systems and large differences between the average power and the peak power, there is a higher arbitrage opportunity for BESS, mainly in costly underground systems.

As a next step, a fuzzy method has been used due to the lack of certainty in how to calculate customer interruption cost in literature. Most of the research in this area is not practical as unreasonable values of customer interruption costs are used for translating SAIDI values in minutes per year to dollars per year. While the proposed algorithm minimizes SAIDI for the upgraded system, it does not depend on the customer interruption cost for optimization. 


\section{CHAPTER 3}

\section{A FuZzy OPTIMIZATION MODEL FOR DisTribution}

\section{System Asset Planning With Energy Storage}

Most DSA planning tools fail to include Battery Energy Storage Systems (BESS) as an asset solution for systems with high penetration of renewable energy sources. In addition, minimizing the investment cost and maximizing the reliability performance, which can be measured by the System Average Interruption Duration Index (SAIDI), are contradicting objectives. With a large investment, the system will have a small SAIDI, and vice versa. The distribution system reliability is contrary to customer interruptions, which are quantified by SAIDI [17] and [20]. A single cost function, including customer interruption cost, is used in such works. However, a trade-off always exists between reliability and cost. Use of a single objective may lead the problem into an infeasible zone, resulting in failure to come to a solution, especially for large complex systems.

With the objective of overcoming these limitations and the fact that the amount of SAIDI and the customer interruption cost incorporated with it is always imprecise and subjective, this work presents a new fuzzy logic algorithm that considers two contradicting objectives in different units. It tries to reduce the asset cost in dollars per year while minimizing the SAIDI measured in minutes per year. In such a case, the SAIDI doesn't need to be translated into dollar amount.

In this thesis, a multi-objective algorithm that uses fuzzy optimization technique to handle these contradicting objectives is proposed. The algorithm includes traditional types of assets as well as 
energy storage systems. One of the main advantages of the proposed method is that the Customer Interruption Costs are not explicitly necessary, as these values cannot be precisely determined for all loads. In the tool, the annual investment cost and SAIDI are minimized, and the optimal size of the energy storage is obtained. Its performance is demonstrated on a 33-bus distribution system and the results are compared with other method available in the literature and the results are discussed in the following sections.

\subsection{Fuzzy Optimization}

Traditional optimization techniques and methods have been successfully applied for years to solve problems with a well-defined structure/configuration, sometimes known as hard systems. Such optimization problems are usually well formulated by crisply specific objective functions and specific systems of constraints, and solved by precise mathematics. Unfortunately, real world situations are often not deterministic. There exist various types of uncertainties in social, industrial and economic systems, such as randomness of occurrence of events, imprecision and ambiguity of system data and linguistic vagueness, which could be caused by errors of measurement, deficiency in history and statistical data, insufficient theory, incomplete knowledge expression, and the subjectivity and preference of human judgement. Such types of uncertainty are categorized as fuzziness. It deals with the situation where the information cannot be valued sharply. This type of fuzziness is usually represented by membership functions which reflect the decision-maker's subjectivity and preferences on the objects. This method is useful when a problem cannot be formulated and solved effectively by traditional mathematics-based optimization techniques nor

probability-based stochastic optimization approaches. However, fuzzy set theory which was 
developed by Zadeh in the 1960's and fuzzy optimization techniques provide a useful and efficient tool for modelling and optimizing such systems. Modelling and optimization under a fuzzy environment is called fuzzy modelling and fuzzy optimization.

\subsection{Problem Formulation}

This thesis proposes a method, where the problem is modeled as a multi-objective optimization model with contradicting objectives of SAIDI minimization and total cost minimization. Fuzzy optimization was used to handle these multiple objectives, which cannot be directly combined into a single objective function without using hard to estimate CIC (customer interruption cost) values. This method has value due to the lack of certainty in how to calculate the CIC values, mainly in residential feeders. Indeed, most of the research in this area has been conducted by using the CIC values to translate SAIDI values from minutes per year to dollars per year. In the proposed method, this is not necessary, as the algorithm minimizes SAIDI directly for the upgraded system. This section introduces the formulation of the proposed algorithm that has a multi-objective function and is capable of planning for traditional assets such as feeders and transformers with the incorporation of new BESS units while optimizing the size and the location of the BESS. The algorithm also determines a plan life, based on consideration of the life of various assets. The total cost comprises the costs of new and replacement assets and the costs of operation and maintenance. SAIDI is the system average for interruption of supply to customers measured in minutes per year. To ensure the practical application of the solution, the formulation is constrained by limits on bus voltages, feeder capacities, BESS unit capacities and the bus-power balance equations. The objective function and its constraints are given below. 


\subsubsection{Objective Function}

In this work, fuzzy optimization technique is used. The multiple objectives of conventional multiobjective challenge are translated into fuzzy satisfaction functions, one for each objective. This allows expression of each objective on its own scale without the requirement of conversion to common units. Thereafter, the minimum of these satisfaction functions is maximized.

In other words, the objective function maximizes the satisfaction of fuzzy functions of objective functions of total cost and SAIDI without requirement for conversion.

\subsubsection{Total Cost Calculation}

TC(x) comprises feeder costs, BESS unit costs, BESS unit replacement costs, feeder maintenance costs, and BESS maintenance costs as set out below, $N Y$ is a variable whose value is optimally determined as part of the solving of this formulation. Although the following equations are identical to the ones in Chapter 2, the description is repeated for each equation:

$$
T C(x)=f_{S F}(\overline{S F}, N Y)+f_{B}(\overline{S B}, N Y)+f_{r e p B}(\overline{S B}, N Y)+f_{m c F}(N Y)+f_{m c B}(N Y)
$$

In the above equation, the total cost comprises of feeder costs, BESS unit costs, BESS unit replacement costs, feeder maintenance costs and BESS maintenance costs.

The annualized costs of new feeder sections are computed as follows:

$$
f_{S F}(\overline{S F}, N Y)=\frac{r(1+r)^{N Y}}{(1+r)^{N Y}-1} \cdot \sum_{l=1}^{N L} K F_{l} \cdot\left(N C_{l}\right) \cdot \overline{S F_{l}}
$$


where $K F_{l}$ is the feeder cost constant, $N C_{l}$ is the number of feeder circuits, and $\overline{S F_{l}}$ is the peak flow of the feeder.

The annualized cost of the BESS units is calculated as follows:

$$
f_{B}(\overline{S B}, N Y)=\frac{r(1+r)^{N Y}}{(1+r)^{N Y}-1} \cdot \sum_{i=1}^{N X}\left(K B P \cdot \overline{P B_{\imath}}+K B E \cdot \overline{P B_{l}}\right)
$$

where $K B P$ and $K B E$ correspond to the cost constants for power and energy respectively of the BESS units, and $\overline{P B_{\iota}}$ and $\overline{E B_{\iota}}$ are the respective optimized power and energy capacities of the BESS unit at bus $i$.

$N R$ represents the number of replacements, and $B Y$ indicates the useful life time of one BESS unit:

$$
N R=N Y / B Y
$$

The annualized cost of replacing the BESS units is then given as:

$$
f_{r e p B}(\overline{S B}, N Y)=\frac{r(1+r)^{N Y}}{(1+r)^{N Y}-1} \cdot \sum_{i=1}^{N X} \sum_{n=1}^{N R} \frac{\left(K B P R \cdot \overline{P B_{l}}+K B E R \cdot \overline{E B_{l}}\right)}{(1+r)^{n \cdot B Y}}
$$

where $K B P R$ and $K B E R$ correspond to the replacement cost constants for BESS unit power and energy, respectively.

The annualized feeder maintenance cost is as follows:

$$
f_{m c f}(N Y)=\frac{r(1+r)^{N Y}}{(1+r)^{N Y}-1} \cdot \sum_{n=1}^{N Y} \frac{K_{m f 0}\left(1+K_{m f}\right)^{n}}{(1+r)^{n}}
$$

where $K_{m f 0}$ is the present initial maintenance cost of the feeders, and $K_{m f}$ is the growth rate for maintenance costs.

Considering maintenance for the BESS unit and its replacement, the annualized maintenance cost of the BESS units is calculated as follows: 


$$
f_{m c B}(N Y)=\sum_{i=0}^{N R-1} \sum_{n=1}^{B Y-1} \frac{K_{B f}\left(1+K_{B v}\right)^{n}}{(1+r)^{n+i \cdot B Y}}+\sum_{n=1}^{\substack{N Y-\\ N R * B Y}} \frac{K_{B f}\left(1+K_{B v}\right)^{n}}{(1+r)^{n+N R \cdot B Y}}
$$

where $K_{B f}$ is the initial present maintenance cost of the BESS units, and $K_{B v}$ is the growth rate for maintenance costs. The first part of the equation deals with the maintenance cost of BESS and the second part of (3.7) calculated the maintenance cost of the BESS replacement.

\subsubsection{SAIDI Minimization}

The $\mathrm{SD}(\mathrm{X})$ in (3.8) stands for System Average Interruption Duration Index (SAIDI). Originally if a feeder is always lightly loaded, then it provides the greatest reliability with the lowest customer interruption costs. However, feeders that are heavily loaded at all times are associated with the lowest reliability levels and the highest customer interruption duration. This phenomenon can be investigated by computing the reliability at each loading level, and then multiplying the result by the probability of the feeder being at that loading level to obtain a weight for each result. This calculation is then repeated for each feeder, ranging from light-peak loading to heavy-peak loading. The final result indicates the relationship of the level of reliability, expressed in terms of SAIDI, to the ratio of the peak load divided by the feeder capacity. The customer interruption cost can then be estimated from the computation of the SAIDI value for a feeder section as a function of the peak load. The SAIDI-per-feeder-circuit value can be computed as follows, based on [65]. (More detailed explanation in Appendix A):

$$
\mathrm{SD}(X)=\frac{A\left(\frac{\max _{t}\left\{S F_{l t}\right\}}{N C_{l} \cdot \overline{S F_{l}}}\right)^{2}+B\left(\frac{\max _{t}\left\{S F_{l t}\right\}}{N C_{l} \cdot \overline{S F_{l}}}\right)+C}{N C_{l}} \times N L \times N Y
$$


It is known historically that the reliability of residential branches is not highly sensitive to loading. This is because the branch is heavily loaded only for a few hours per year. The situation is different for commercial/industrial (C\&I) feeders. However, with the emergence of Electric Vehicles, some of the residential branches are heavily loaded for longer periods.

\subsubsection{Fuzzy Set of Objective Function}

Fuzzy satisfaction functions help describe satisfaction of an objective. Considering multiple objectives, the fuzzy optimization technique determines the minimum of all satisfaction functions and maximizes it. The satisfaction functions for the conflicting objectives are defined between 0 and 1 as shown in Fig. 3.1 and Fig. 3.2 for Total Cost $(T C(x))$ and SAIDI $(S D(x))$, respectively.

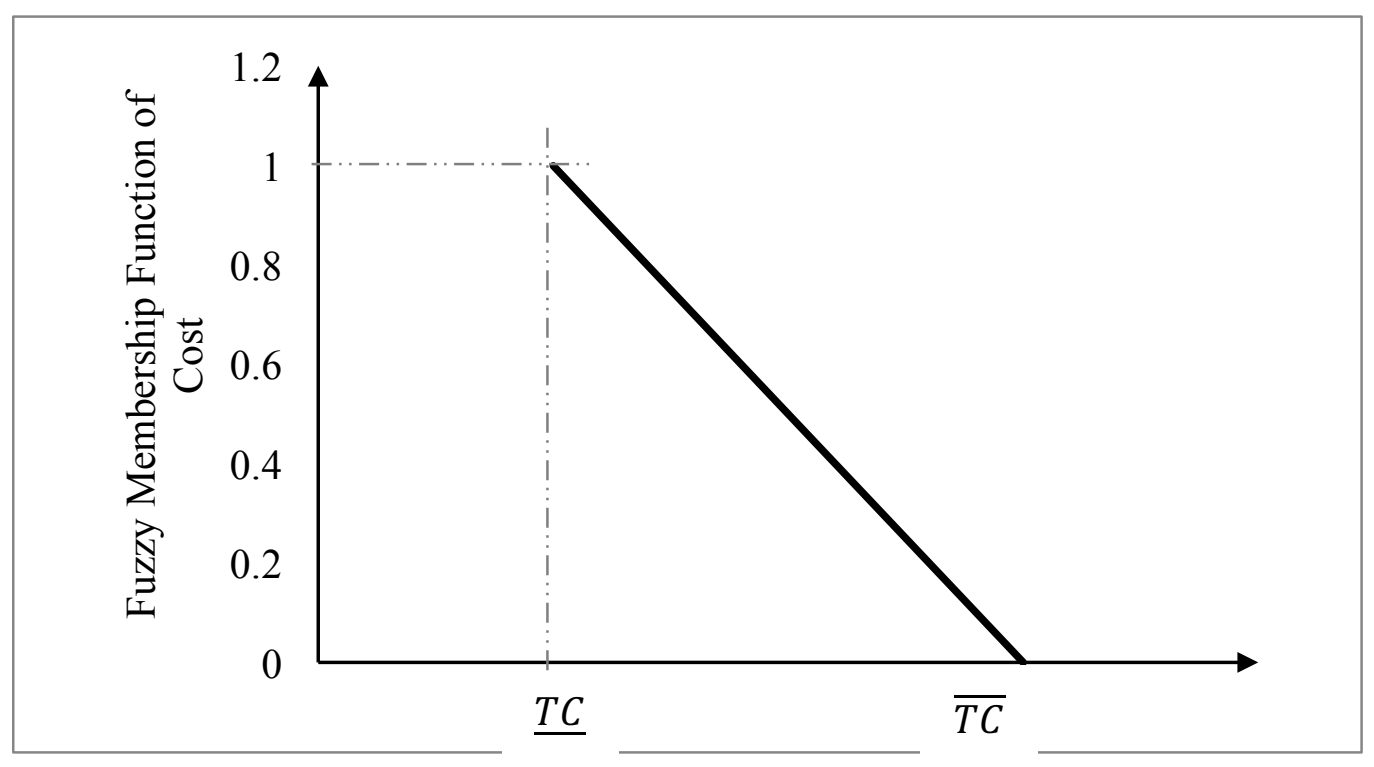

Fig. 3.1 Fuzzy Satisfaction Functions of Total Cost (TC) 


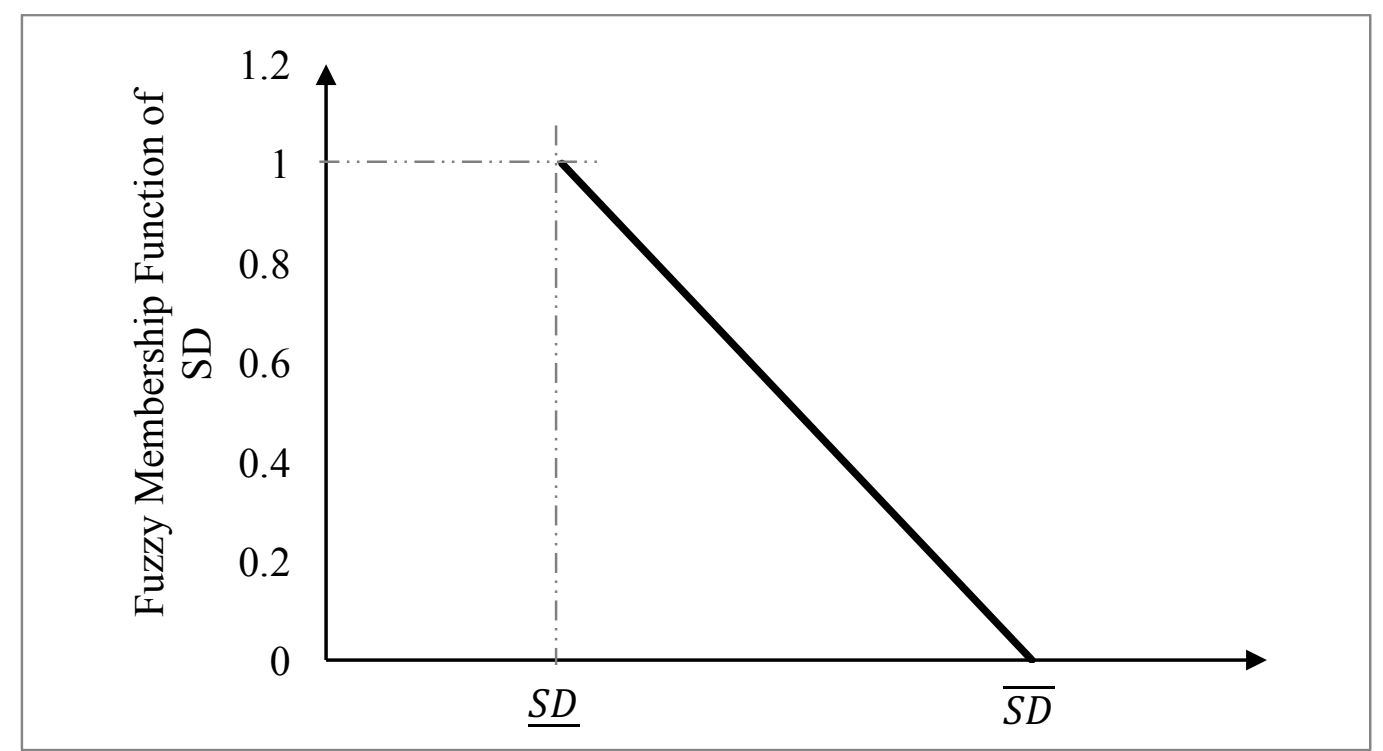

Fig. 3.2 Fuzzy Satisfaction Functions of SAIDI (SD)

$\mu_{T C}(X)$ is a fuzzy member for total cost $T C(x)$ and is shown in (3.9):

$$
\mu_{T C}(X)=\frac{\overline{T C}-T C(X)}{\overline{T C}-\underline{T C}}
$$

where $\overline{T C}$ and $\underline{T C}$ are maximum and minimum values of $T C(x)$ described in (3.1).

Also with the definition of $\mathrm{SD}(\mathrm{X})$, the fuzzy satisfaction function for SAIDI can be defined as below. $\mu_{S D}(x)$ is a fuzzy satisfaction function for SAIDI and is shown in (3.10):

$$
\mu_{S D}(x)=\frac{\overline{S D}-S D(X)}{\overline{S D}-\underline{S D}}
$$

where $\overline{S D}$ and $\underline{S D}$ are maximum and minimum values of $S D(X)$ described in (3.8). 


\subsubsection{Objective Definition}

The multi-objective function is limited by a set of rules and constraints that are explained below. The satisfaction function for the objective is defined as: $\mu_{O B J}$

This is lesser than or equal to all other satisfaction functions as it equals the least of all satisfaction functions.

Therefore:

$$
\begin{aligned}
& \mu_{O B J} \leq \mu_{T C} \\
& \mu_{O B J} \leq \mu_{S D}
\end{aligned}
$$

The final objective function is then defined as below:

$$
\text { Objective: } \operatorname{Max} \mu_{O B J}
$$

The objective (3.13) searches for the maximum $\mu_{O B J}$ which is a contradicting combination of $\mu_{T C}$ or $\mu_{S D}$ while keeping them independent from each other. In Fig. 3.1 and Fig. 3.2, the algorithm finds the minimum values of $\mu_{T C}$ and $\mu_{S D}$ while maximizing the objective function pushes those values to be as close as possible to $\underline{T C}$ and $\underline{S D}$.

\subsubsection{Constraints and Boundaries}

All the equations in this section are identical to Chapter 2 but are repeated here to help the flow of the thesis. (More detailed explanation in Appendix A).

Since the feeders are in parallel, the new upgraded line admittance is equal to the previous admittance multiplied by the number of upgraded feeders:

$$
y_{l l}=y_{l} \cdot N C_{l}
$$


The complex bus-wise power balance equation is computed for time $t$ as follows:

$$
S D_{i t}+S L_{i t}\left(V_{i t}, \delta_{i t}, y_{l l}\right)-S G_{i t}-S B_{i t}=0
$$

where $S G$ is the vector of the bus-wise apparent power generation, $S D$ is the vector of the bus-wise apparent power loads, and $S L(V, \delta)$ is the vector of the apparent power injected from the buses into the connected lines. $S B$ is the vector of the apparent power of the BESS units. (For more detailed derivation, refer to equations A.1 and A.2 in Appendix A).

The power flow in feeder segment $l$ between buses $i$ and $j$ at time $t$ is computed as follows:

$$
S F_{l}-V_{i t} \angle \delta_{i t} \cdot\left[\left(V_{i t} \angle \delta_{i t}-V_{j t} \angle \delta_{j t}\right) \cdot y_{l l} \angle \theta_{l}\right]^{*}=0
$$

The energy in BESS unit $i$ at time $t$ can be computed as follows:

$$
E B_{i t}=H T_{t} \cdot \max \left(P B_{i t} \cdot K_{P} \frac{P B_{i t}}{K_{P}}\right)+E B_{i, t-1} \cdot K_{E}
$$

where $K_{P}$ models the efficiency of the power conversion system, and $K_{E}$ accounts for any selfdischarge.

In addition, the sum of the energy stored and retrieved from a BESS unit is constrained to equal zero over the study period, with $P B$ being the vector of the active power of the BESS unit.

$$
\sum_{t=1}^{N H} H T_{t} \cdot P B_{i t}=0 ; i=1,2 \ldots N X
$$

The formulation is also subject to the following boundaries:

Limits on the bus voltage magnitudes:

$$
\underline{V} \leq V_{i t} \leq \bar{V}
$$

Limits on complex power generated by the sources: 


$$
\underline{S G_{i}} \leq S G_{i t} \leq \overline{S G_{i}}
$$

BESS power limits:

$$
\underline{S B} \leq S B_{i t} \leq \overline{S B} \quad i=1,2 \ldots N X
$$

Complex line flow limits:

$$
\left|S F_{l t}\right| \leq \overline{S F_{l}}
$$

Solving the optimization formulation (3.1) to (3.13), based on the above constraints (3.14) to (3.22), enables the minimization of the annualized investment cost and the determination of the optimal number of years for the best asset life while minimizing SAIDI.

\subsection{Solution Algorithm}

As previously mentioned, the proposed planning formulation and algorithm includes a multiobjective function and it chooses the best planning option using the list of traditional assets and also BESS. Optimally sizing the BESS units makes the results more practical. It is important to clarify that the proposed model is a Mixed Integer Nonlinear problem (MINP) using OPTI Toolbox in MATLAB Programming language. This optimization method was implemented using interior-point algorithm method and it has Fuzzy Logic structure. 


\subsection{Results and Discussion}

The analyzed systems are undergrounded urban systems with critical infrastructure for which customer interruption costs are significant, although for the proposed algorithm, it is not necessary to assign a value to customer interruption cost. This will yield more accurate results, as all the studies that have calculated the SAIDI amount based on customer interruption cost are either outdated or have used wide approximations in their method.

\subsubsection{Economic Data}

The economic data (Table 3.1) used for this work is identical to the Table 2.1 in Chapter 2, only without the Customer Interruption Cost. Both tables are based on the Final Report by InfraSource Technology [66].

Table 3.1 Feeder and BESS Costs (US\$)

\begin{tabular}{l|l}
\hline \multicolumn{1}{c|}{ Item } & \multicolumn{1}{c}{ Value } \\
\hline$K F-$ Underground Feeder cost coefficient (\$/MVA) & $\$ 1,350,000$ \\
\hline$K B E$ - Energy cost coefficient for a battery (\$/MWh) & $\$ 90,000$ \\
\hline$K B P$ - Power cost coefficient for a battery (\$/MW) & $\$ 300,000$ \\
\hline Underground feeder maintenance cost (\$/year) & $\$ 9,000$ \\
\hline BESS maintenance cost (\$/year) & $\$ 1,000$ \\
\hline BESS replacement period (years) & 10 \\
\hline Replacement Cost for Battery Power Capacity (\$/MW) & $\$ 210,000$ \\
\hline Replacement Cost for Battery Energy Capacity (\$/MWh) & $\$ 70,000$ \\
\hline Interest rate (per year) & $5 \%$ \\
\hline
\end{tabular}




\subsubsection{IEEE 33-Bus Distribution System Data}

The proposed method was validated through implementation on the IEEE 33-bus test system shown in Fig. 3.3. The detailed data for the 33-bus radial distribution system is in Appendix B. The feeder capacity for the system is assumed to be 5 MVA. The load active and reactive values as well as the feeder data are taken from [68].

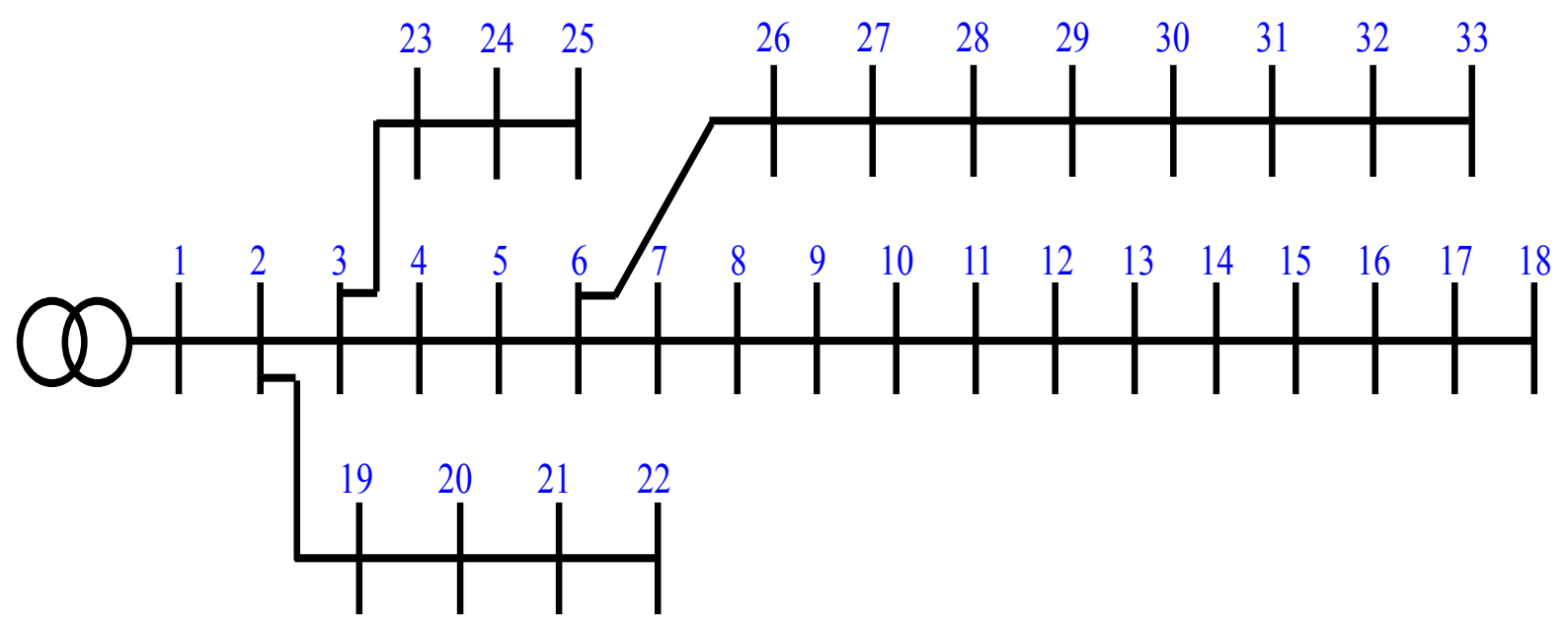

Fig. 3.3 IEEE 33-Bus System

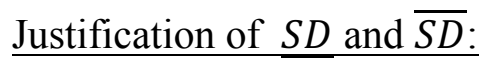

The $\underline{T C}$ and $\overline{T C}$ are set to be respectively $\$ 0$ and $\$ 200,000$ and the $\underline{S D}$ and $\overline{S D}$ are set to be respectively 512 and 1,036 hours.

At $0 \%$ loading, SAIDI is at a minimum and using (9) and multiplying the number of SAIDI to the number of branches $(\mathrm{NL}=32)$ and number of years $(\mathrm{NY}=20)$ the feeders are assumed to last under $100 \%$ loading condition, one can calculate $\underline{S D}$ as follows: 


$$
\underline{\mathrm{SD}}=\frac{A\left(\frac{0}{N C_{l} \cdot \overline{S F_{l}}}\right)^{2}+B\left(\frac{0}{N C_{l} \cdot \overline{S F_{l}}}\right)+C}{N C_{l}} \times N L \times N Y=512 \text { hours }
$$

Therefore, $\underline{S D}$ equal to 512 hours and is used for analysis of the proposed method.

On the other hand, at $100 \%$ loading, $\overline{S D}$ is being calculated in the following manner, assuming number of circuits is equal to 1 and emergency rating is 1.5 :

$$
\overline{\mathrm{SD}}=\frac{A\left(\frac{1}{1 * 1.5}\right)^{2}+B\left(\frac{1}{1 * 1.5}\right)+C}{1} \times N L \times N Y=1036 \text { hours }
$$

In the proposed algorithm $\overline{\mathrm{SD}}$ has been 1036 hours as the maximum SAIDI.

To check the method performance, the load scenarios were divided in three zones as follows:

•40 \% peak load (as a light load) - Time Zone 1 (5 hours)

•140\% peak load (Very heavy load) - Time Zone 2 (6 hours)

-90 \% peak load - Time Zone 3 (13 hours)

The load and generation values for each region are provided in Table 3.2, where PD stands for Power Demand.

Table 3.2 System Power Demand (PD) Values for Each Time Zone

\begin{tabular}{c|c|c|c}
\hline & Time Zone 1 & Time Zone 2 & Time Zone 3 \\
& 5 hours & 6 hours & 13 hours \\
\hline PD & 1.4860 & 5.2010 & 3.3435 \\
$(\mathrm{MW})$ & $(40 \%$ peak load $)$ & $(140 \%$ peak load $)$ & $(90 \%$ peak load $)$ \\
\hline
\end{tabular}


Then the algorithm solves the planning for two different cases. For each case, the proposed method establishes the optimal plan that results in the lowest total amortized annual cost solution.

- Case I: Conventional planning model that considers only feeders (i.e., no BESS units)

- Case II: Proposed planning model that considers feeders and BESS units.

In case I (no BESS), the loading on the feeders is higher, and the lowest estimated life cycle cost is US\$ 902,009 which is composed of the following costs:

Annualized Feeder Upgrade Costs: $\quad$ US\$ 884,900

Annualized Feeder Maintenance Costs: $\quad$ US\$ 17,109

Total annualized Costs: $\quad$ US\$ 902,009

The results of this case are documented in Table 3.3 and indicate that two additional circuit feeders are needed to eliminate the overload of Sections \#1 and \#2 during Time Zone 2. Also, the total SAIDI is 902.0446 hours for the whole planning period. The fuzzy satisfaction factors, $\mu_{T C}$ and $\mu_{S D}$ are calculated to be 0.0980 and equal.

In case II (with BESS), an optimal plan is developed whereby no additional feeders are necessary and only one BESS unit is sized, as one can see in Table 3.4 and Table 3.5 The fuzzy satisfaction values, $\mu_{C}$ and $\mu_{\mathrm{S}}$ are 0.3523 and the total SAIDI for the length of planning years is 647.72 hours. 
The minimum cost of the annual asset plan is calculated to be US\$178,700 which is composed of the following costs:

Annualized Feeder Upgrade Costs: $\quad$ US\$ 0

Annualized Feeder Maintenance Costs: US\$ 13,744

Annualized BESS Costs: $\quad$ US\$ 114,413

Annualized Battery Replacement Costs: US\$ 49,187

Annualized Battery Maintenance Costs: US\$ 1,356

Total annualized Costs: $\quad$ US\$ 178,700

Table 3.5 shows the energy and power in the BESS unit. It is optimally sized for $1.45 \mathrm{MW}$ and $10.98 \mathrm{MWh}$.

Table 3.3 Total Line Power Flow for the IEEE 33-Bus System - Case I

\begin{tabular}{|c|c|c|c|c|c|}
\hline \multirow{3}{*}{ Section \# } & \multicolumn{3}{|c|}{ Line Power Flow (MVA) } & \multirow{3}{*}{$\begin{array}{l}\text { Feeder } \\
\text { Capacity } \\
\text { (MVA) }\end{array}$} & \multirow{3}{*}{$\begin{array}{l}\text { Maximum \# of } \\
\text { Feeders } \\
\text { Required }\end{array}$} \\
\hline & Time & Time & Time & & \\
\hline & Zone 1 & Zone 2 & Zone 3 & & \\
\hline 1 & 1.7523 & 6.1738 & 3.9557 & 5 & 2 \\
\hline 2 & 1.5495 & 5.4625 & 3.4989 & 5 & 2 \\
\hline $3-32$ & $<5$ & $<5$ & $<5$ & 5 & 1 \\
\hline
\end{tabular}


Table 3.4 Total Line Power Flow for the IEEE 33-Bus System - Case II

\begin{tabular}{c|c|c|c|c|c}
\hline \multirow{2}{*}{ Section \# } & \multicolumn{2}{|c|}{ Line Power Flow (MVA) } & \multirow{2}{*}{ Feeder } & Maximum \# of \\
\cline { 2 - 4 } & Time & Time & Time & Capacity & Feeders \\
& Zone 1 & Zone 2 & Zone 3 & (MVA) & Required \\
\hline 1 & 2.8671 & 4.9983 & 4.4405 & 5 & 1 \\
\hline 2 & 2.6638 & 4.3101 & 3.9800 & 5 & 1 \\
\hline $3-32$ & $<5$ & $<5$ & $<5$ & 5 & 1 \\
\hline
\end{tabular}

Table 3.5 Battery Power and Energy for the IEEE 33 Bus System - Case II

\begin{tabular}{c|c|c|c}
\hline & Time Zone 1 & Time Zone 2 & Time Zone 3 \\
\hline Battery Power (MW) & -1.2173 & 1.4526 & -0.5498 \\
\hline Battery Energy (MWh) & 11.0474 & 0.0383 & 6.3239 \\
\hline
\end{tabular}

From these results, it is clear that with the use of the proposed planning method, BESS can be investigated as a viable asset for system planning. There are a few advantages in using BESS based on the above results. First, for this case, it is more economical since it is the least costly option. Second, the number of SAIDI is much lower while using a BESS unit. The reason for that is the relaxation of the loading percentage of the feeders in the presence of the BESS during heavy loading. 


\subsubsection{Sensitivity Analysis for the 33-Bus Distribution System}

As was previously mentioned, the proposed algorithm uses fuzzy optimization technique. It provides a means to optimize two conflicting objectives that are on different scales and cannot be combined. In other words, it is not necessary to accurately calculate the cost of interruption, which is a challenging task. The following studies are only possible due to the characteristics of fuzzy sets and are not achievable through any other method.

One of the objectives to be minimized is $\mu_{S D}$ which is explained in (3.10). In this sensitivity Analysis, the value of $\underline{\mathrm{SD}}$ is being varied in Case II (with BESS). The effect of this variation is shown in Fig. 3.4 and Table 3.6.

In this study $\overline{S D}$ remained constant at 1,000 hours and SD increased from 0 to 900 hours. The value of $\mathrm{SD}(\mathrm{X})$ will increase as a result. That happens because the method maximizes (3.13) by getting $\mu_{\mathrm{SD}}$ as close as possible to 1 . According to (3.10) as $\overline{S D}$ and $\underline{\mathrm{SD}}$ becomes closer to each other, the denominator becomes smaller and therefore the value of $\mu_{\mathrm{SD}}$ will become higher. At the same time NY will increase as well, because the number of years depends on the $\operatorname{SD}(\mathrm{X})$. The size and the present cost of the BESS remains unchanged for all the $\underline{\mathrm{SD}}$ values. However, $\mathrm{TC}(\mathrm{X})$ decreases as $\underline{\mathrm{SD}}$ increases. The reason is that since there is not much room to optimize $\mathrm{SD}(\mathrm{X})$, the objective function puts $\mathrm{TC}(\mathrm{X})$ as priority and starts minimizing it by adding more years to the project. It should be mentioned that the BESS units must be replaced every 10 years, which is the reason behind the discontinuity in the $\mathrm{TC}(\mathrm{X})$ at year 13 and year 23. Basically, after the year 10 and the year 20, the replacement cost of BESS has been added to the costs. 


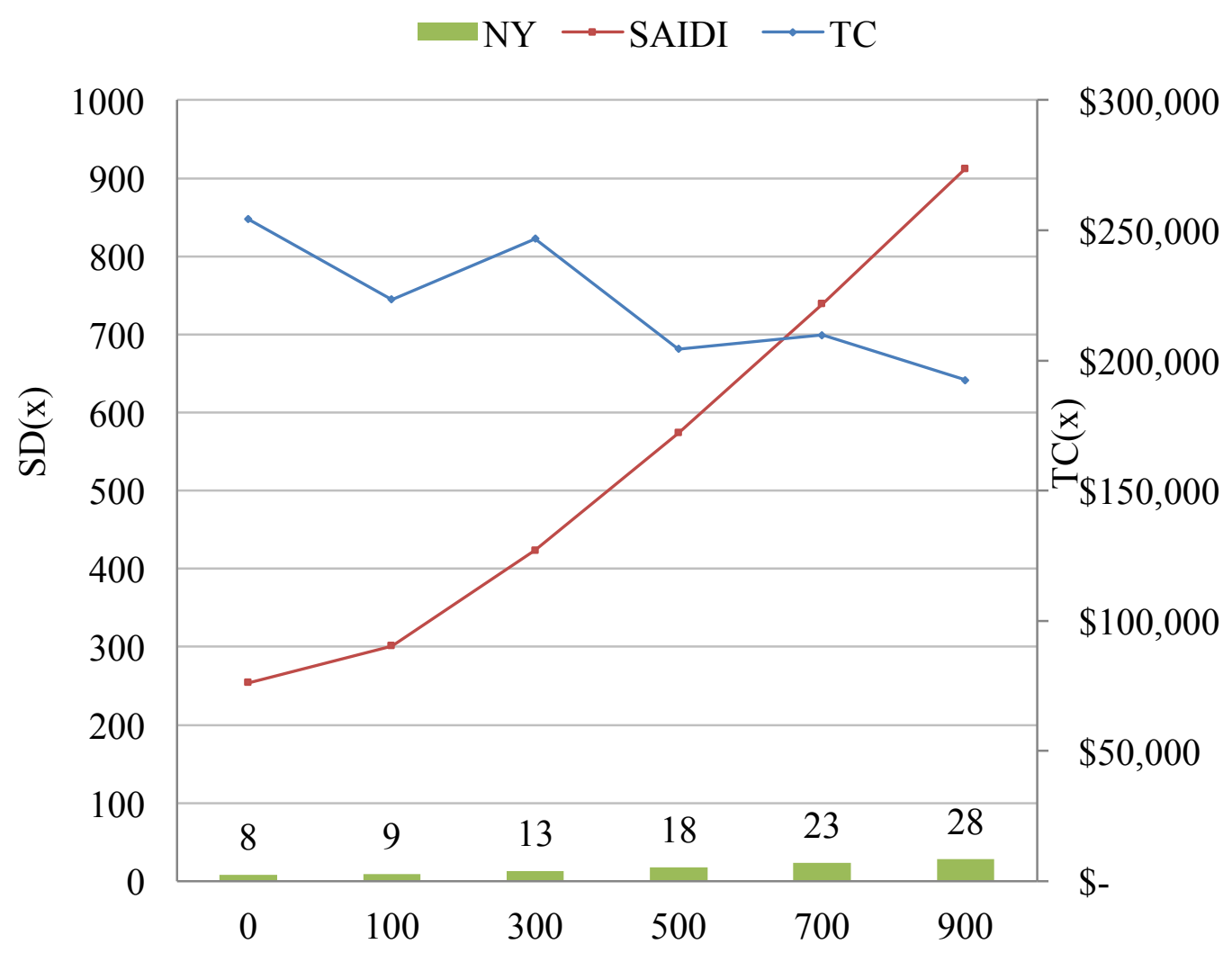

Fig. 3.4 Sensitivity Analysis Based on SD Variation

Table 3.6 Sensitivity Analysis on $\underline{\text { SD }}$ for 33-bus System

\begin{tabular}{c|c|cc|c|c}
\hline$\underline{\mathrm{SD}}$ & $\mathrm{SD}(\mathrm{X})$ & \multicolumn{2}{|c|}{$\mathrm{TC}(\mathrm{X})$} & $\mathrm{NY}$ & $\mu_{\mathrm{SD}} \& \mu_{\mathrm{TC}}$ \\
\hline 0 & 254.1893 & $\$$ & 254,200 & 8 & 0.7458 \\
\hline 100 & 301.036 & $\$$ & 223,400 & 9 & 0.7766 \\
\hline 300 & 423.6684 & $\$$ & 246,700 & 13 & 0.8233 \\
\hline 500 & 573.6849 & $\$$ & 204,400 & 18 & 0.8526 \\
\hline 700 & 739.1271 & $\$$ & 209,800 & 23 & 0.8695 \\
\hline 900 & 912.0965 & $\$$ & 192,400 & 28 & 0.8789 \\
\hline
\end{tabular}


In the second sensitivity analysis, the $\overline{S D}$ and $\underline{S D}$ remain constant at 1,036 hours and 512 hours, respectively, whereas, the value of $\overline{T C}$ is varied from $\$ 200,000$ up to $\$ 500,000$ with $\underline{T C}$ equal to $\$ 0$. The effects of this variation are shown in Fig. 3.5 and Table 3.7. As $\overline{T C}$ increases from $\$ 200,000$ to $\$ 500,000$, the optimal value of $T C(x)$ increases, so that the solution will become more expensive. That is a consequence of $N Y$ becoming shorter. $S D(x)$ also decreases as $\overline{T C}$ and $T C(x)$ become higher which is due to the nature of fuzzy method and two contradicting objectives of the algorithm. This study is specifically essential for the distribution companies that have tighter limits on their yearly capital expenditures.

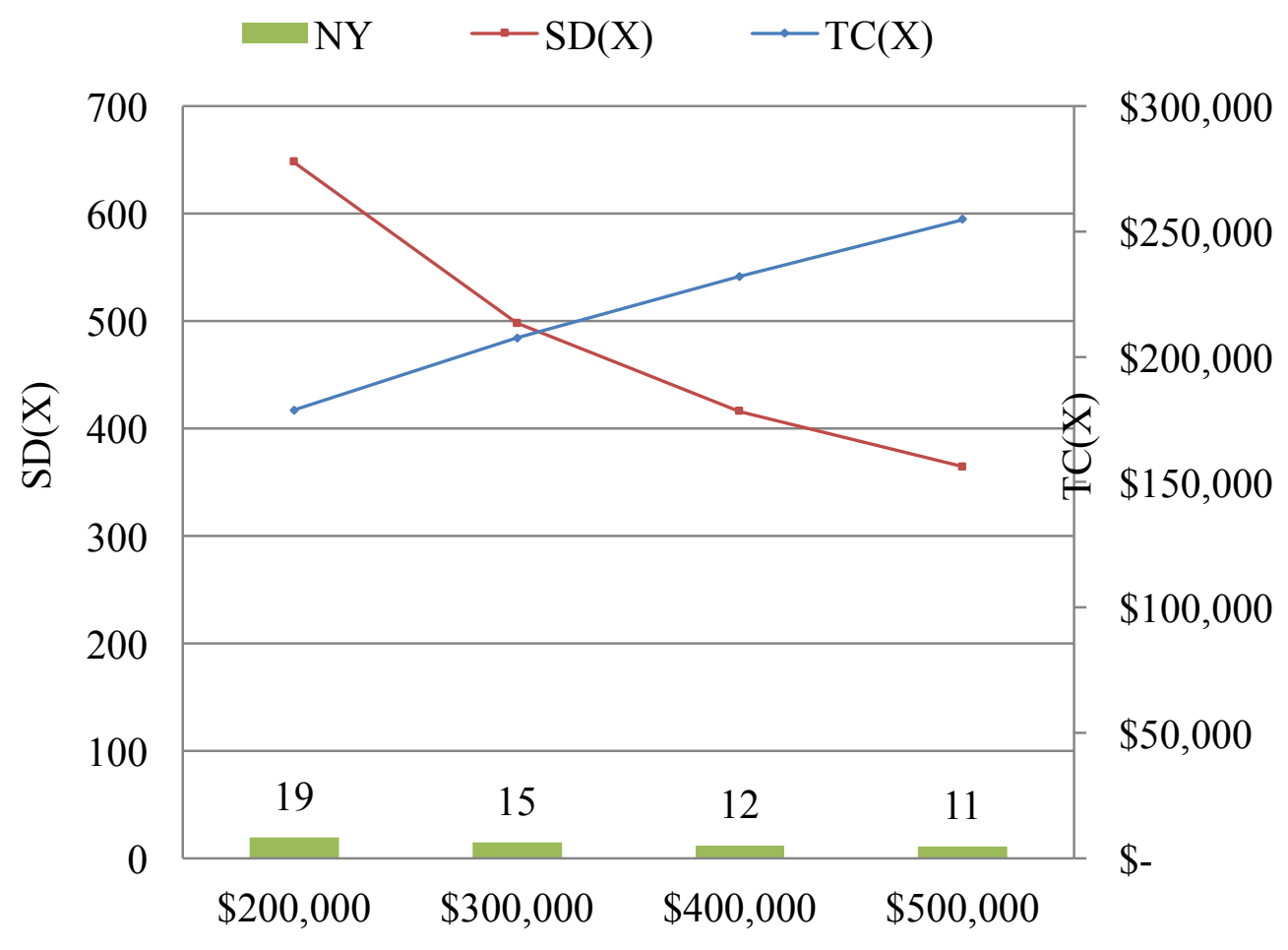

Fig. 3.5 Sensitivity Analysis Based on $\overline{T C}$ Variation 
Table 3.7 Sensitivity Analysis on $\overline{T C}$ for 33-bus System

\begin{tabular}{ll|c|cc|c|c}
\hline & $\overline{T C}$ & $\mathrm{SD}(\mathrm{X})$ & \multicolumn{2}{c|}{$\mathrm{TC}(\mathrm{X})$} & $\mathrm{NY}$ & $\mu_{\mathrm{SD}} \& \mu_{\mathrm{TC}}$ \\
\hline$\$$ & 200,000 & 647.7239 & $\$$ & 178,700 & 19 & 0.3523 \\
\hline$\$$ & 300,000 & 497.8992 & $\$$ & 207,500 & 15 & 0.5021 \\
\hline$\$$ & 400,000 & 415.6073 & $\$$ & 232,100 & 12 & 0.5844 \\
\hline$\$$ & 500,000 & 364.1388 & $\$$ & 254,700 & 11 & 0.6359 \\
\hline
\end{tabular}

\subsubsection{Sensitivity Analysis for the 33-Bus Distribution System}

In this section, a comparison between the proposed method and one method recently published in literature is presented. The algorithm used for comparison is described in [22] and [25], which will be called Method 2. The annualized upgrade cost determined by both methods are shown in Table 3.8.

As one can see, the result obtained by the proposed method is slightly below the one determined by Method 2, as the allocated battery is slightly smaller. However, the main advantage of the proposed method is that the exact Customer Interruption Cost is not necessary, as SAIDI information is included as a fuzzy satisfaction function. This is relevant as this type of information is not readily and accurately available for the distribution planning engineers, mainly when dealing with residential feeders. 
Table 3.8 Result Comparison - 33-Bus System

\begin{tabular}{c|c|c}
\hline Method & $\begin{array}{c}\text { Battery Power (MW)/ } \\
\text { Energy (MWh) }\end{array}$ & $\begin{array}{c}\text { Total Annualized } \\
\text { Upgrade Cost }\end{array}$ \\
\hline Proposed Method with & $\begin{array}{c}1.45 \mathrm{MW} \\
10.98 \mathrm{MWh}\end{array}$ & $\$ 178,700$ \\
BESS & $1.45 \mathrm{MW}$ & $\$ 180,030$ \\
\hline Method 2 & $10.99 \mathrm{MWh}$ & \\
\hline 22$] \&[25]$ & & \\
\hline
\end{tabular}

\subsection{Chapter Summary}

Besides a technical solution, the usage of Battery Energy Storage Systems (BESS) to enable high integration of renewable energy sources (e.g., solar and wind generation) and new loads (e.g., electric vehicles) is an economic question. This possibility must be included into Distribution System Asset (DSA) planning methods. To contribute to this issue, this thesis proposed a method, where the problem was modeled as a multi-objective optimization model with contradicting objectives of SAIDI minimization and total cost minimization. Fuzzy optimization was used to handle these multiple objectives, which cannot be directly combined into a single objective function without using hard to estimate CIC (customer interruption cost) values. This method has value due to the lack of certainty in how to calculate the CIC values, mainly in residential feeders. Indeed, most of the research in this area has been conducted by using the CIC values to translate SAIDI values from minutes per year to dollars per year. In the proposed method, this is not necessary, as the algorithm minimizes SAIDI directly for the upgraded system. The algorithm can plan for traditional assets such as feeders and transformers as well as the incorporation of BESS units. The 
algorithm was successfully validated on the IEEE 33-bus distribution system and the results were compared with a recent method published in the technical literature and shown to be superior. As the technology advances, the cost of BESS is expected to decrease in the following years, so that the proposed model can be useful especially in urban centers, where the installation of new feeders is costly.

To address operational aspects of distribution systems and minimizing losses, a complementarity method for reconfiguration of distribution systems in real-time is proposed in the next chapter. 


\section{Chapter 4}

\section{REAL-TIME SMART DiSTRIBUTION SYSTEM}

\section{RECONFIGURATION USING COMPLEMENTARITY}

Assuming that a new distribution system has been planned considering the least cost option, how to optimally operate this distribution system using a reliable optimization algorithm is discussed in this chapter. In this research, a new algorithm that can accomplish this task is proposed.

In North America, most power distribution systems are designed and operated as radial circuits. The radial circuit has a single power source feeding a network of downstream feeders.

Smart Radial Distribution Systems of the future will have improved reliability, performance and flexibility in operation by using SCADA operable breakers and algorithms such as optimal reconfiguration in real-time in their distribution management systems while considering the protection design limitations. However, today, optimal reconfiguration algorithms are largely academic because of challenges such as: they depend upon heuristic techniques that require repeated runs and are not suitable for real-time applications, they do not guarantee an optimal solution, and finally, they do not provide insight into solution space.

Electric utilities may reconfigure their network such that total system losses are minimized and voltage profile is satisfactory. Optimal reconfiguration requires that one breaker or tie switch in 
every loop is opened, such that the set of closed breakers results in a radial topology which results in minimum losses and a satisfactory voltage profile. An optimization algorithm for optimal reconfiguration shall consider all possible combinations and scenarios where one switch can be opened in every loop. This problem is a combinatorial challenge and its complexity increases exponentially with the number of switches. Further, examining the solution domain, which is undertaken in the next section, it is evident that the solution space is discontinuous.

Literature review shows that many researchers have attempted different optimization techniques and have found the optimal solution for published examples. However, limitations in classical linear and nonlinear optimization techniques preclude them from being successfully used for this optimization challenge. Hitherto, all attempts are restricted to optimizing using heuristic techniques such as evolutionary programming, genetic algorithms, etc.

To realize smart radial distribution system of the future, a real-time optimal reconfiguration algorithm is proposed, which uses a classic nonlinear optimization technique and guarantees an optimal solution in the least time. The method is based upon a complementarity technique that transforms discontinuous solution spaces into continuous, enabling use of classical nonlinear optimization techniques without resorting to heuristics.

This is successfully demonstrated on 7-bus, IEEE 33-bus and IEEE 69-bus distribution systems. The results are compared with those available in literature with respect to solution time, accuracy in results and robustness of the proposed algorithm and demonstrate the superiority of the proposed technique.

\subsection{Problem Formulation without Complementarity}


The formulation commonly used for reconfiguration without complementarity technique is as follows:

$$
\Psi M_{i}=1 \text { or } 0, \text { for } i=1,2 \ldots N
$$

When a branch is switched in and another is switched out in a loop, the solution space is no longer continuous. The variable $\Psi M_{i}$ that defines the status of a switch as to whether it is open/close assumes discrete states of zero or one.

The following constraint confirms that the system stays radial at all times:

$$
\sum_{i=1}^{N} \Psi M_{i}=N L-N B+1
$$

Total power loss of the network is the summation of all bus power injections. Minimizing this summation is the objective function:

$$
\text { Minimize: } P_{\text {Loss }}=\sum_{i=1}^{N B} P_{L}(V, \delta)
$$

The network is presented by calculating the $\mathrm{Y}_{\mathrm{B}}$ :

$$
\mathrm{Y}_{\mathrm{B}}=\left[\mathrm{Y}_{\mathrm{I}}\right] \cdot[\mathrm{I}-[\Psi \mathrm{M}]] \cdot\left[\mathrm{Y}_{\mathrm{L}}\right] \cdot\left[\mathrm{Y}_{\mathrm{I}}\right]^{\mathrm{T}}
$$

Using the Y-BUS matrix, the power balance equations for buses can be written as follows:

$$
\begin{array}{r}
\mathrm{P}_{\mathrm{G}}-\mathrm{P}_{\mathrm{D}}-\mathrm{P}_{\mathrm{L}}\left(\mathrm{V}, \delta, \mathrm{Y}_{\mathrm{B}}\right)=0 \\
\mathrm{Q}_{\mathrm{G}}-\mathrm{Q}_{\mathrm{D}}-\mathrm{Q}_{\mathrm{L}}\left(\mathrm{V}, \delta, \mathrm{Y}_{\mathrm{B}}\right)=0
\end{array}
$$


The problem has a discontinuous solution space since switches assume only discrete values of zero or one. Therefore, classical optimization methods are not suitable for the following formulation and researches were limited to use heuristic methods.

\subsection{Complementarity}

In this work, in search of making optimal reconfiguration suitable for real-time use, recent work on complementarity [69] and [70] is utilized. Using complementarity, the nonlinear mixed integer optimization challenge of optimal reconfiguration with discontinuous solution space is transformed into a nonlinear optimization challenge with continuous solution space. This transformation allows the use of classical nonlinear optimization solvers that are robust, guaranteeing the optimal solution while being suitable for real-time application.

Accordingly, it is proposed to use complementarity constraints and transform the solution space such that conventional NLP techniques can be used for solving the challenge of optimal reconfiguration of smart radial distribution system in real-time.

Consider the example shown in the problem (P1) with the following objective function:

Maximize:

$$
\left(3 x_{1}-2\right)^{3}+\left(5 x_{2}-1\right)^{3}
$$

Subject to:

$$
\begin{array}{r}
0 \leq x_{i \leq 1} \quad \forall i=1,2 \\
x_{1} \cdot x_{2}=0
\end{array}
$$


The problem is formulated such that only one of the two variables can assume a non-zero value for a feasible solution. Hence, use of classical optimization techniques is not possible. This is true when we model the optimal reconfiguration challenge in distribution systems.

Considering the problem in (4.7) to (4.9), since the result becomes infeasible for a solution other than at points $\left(0,0 \leq x_{2} \leq 1\right)$ and $\left(0 \leq x_{1} \leq 1,0\right)$, the formulation is restated as problem (P2) [69][70]:

Maximize:

$$
\left(3 x_{1}-2\right)^{3}+\left(5 x_{2}-1\right)^{3}-\mathrm{M} \cdot x_{1} \cdot x_{2}(4.10)
$$

The penalty factor, $M$, is an arbitrary large number, which is used to ensure that the multiplication of $\mathrm{x} 1$ and $\mathrm{x} 2$ will be zero. Therefore, solutions which do not satisfy the constraints will be disregarded.

This objective in (4.10) is plotted in Fig. 4.1 with $\mathrm{M}=100$, where it is evident that the optimal solution is $x_{1}=0$ and $x_{2}=1$.

Despite the fact that the $x_{1}$ and $x_{2}$ are both continuous variables limited between 0 and 1 , using complementarity constraints and theory, the result will always yield a discrete solution.

Hence, the problem in (4.7) to (4.9) with discontinuous solution space that could not be handled by classical optimization techniques is now transformed into the form in (4.10) whereby it is continuous and solvable using classical optimization techniques. 


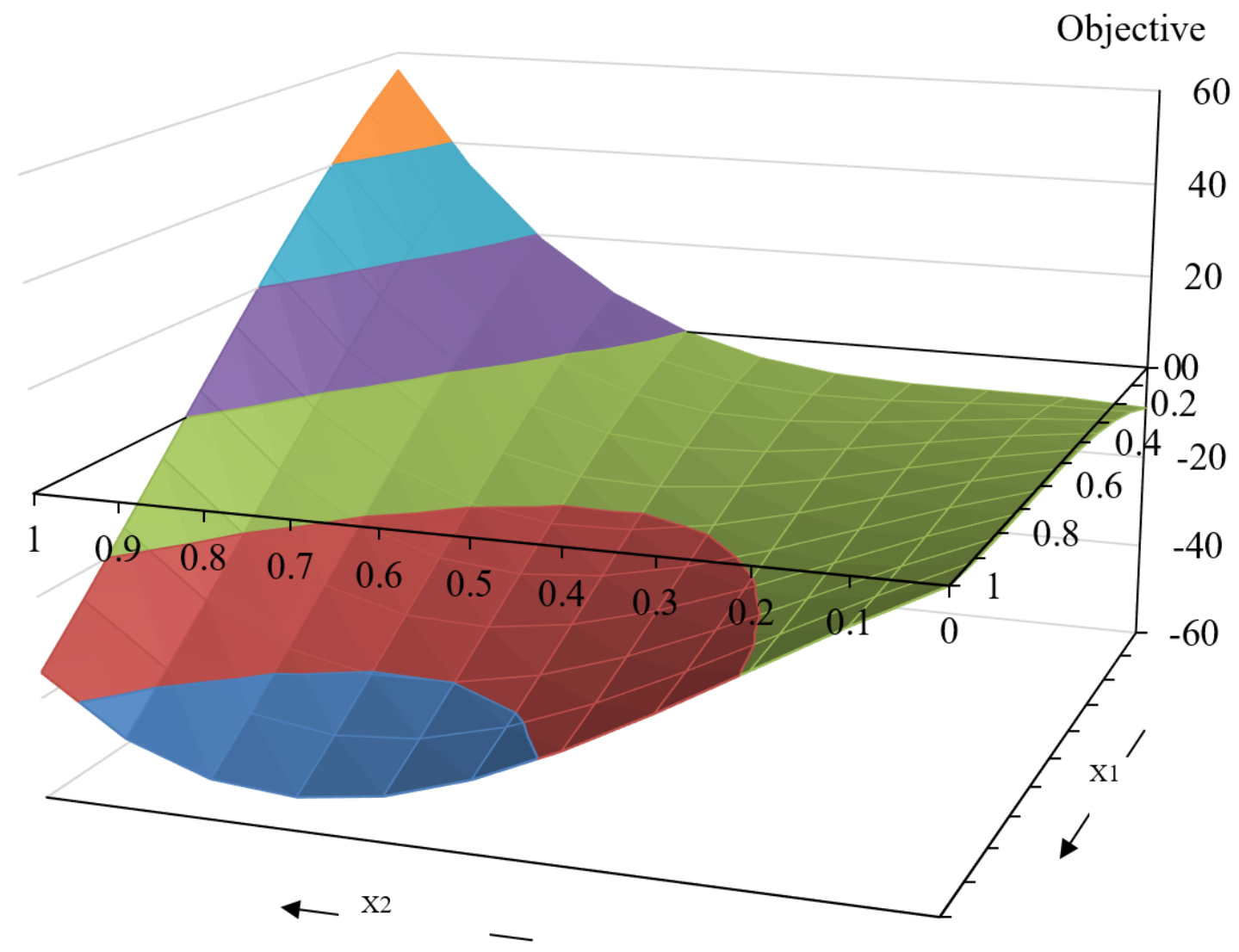

Fig. 4.1 Solution Space for a Sample Problem (P1)

\subsubsection{Switches and Complementarity}

In this work, it is proposed to model the status of switches by continuous variables limited between 0 and 1 , where 0 indicates that the switch is closed and 1 indicates that the switch is open. For the sake of argument, let there be a set of switches in a loop and their status be represented by the continuous variables $\Psi M_{i}$ constrained by:

$$
0 \leq \Psi M_{i} \leq 1, \text { for } i=1,2 \ldots N
$$


If only one switch, $\Psi M_{i}$, can be open in the loop, then complementarity constraints dictate that the following must be true for a feasible solution:

$$
\Psi M_{i} \cdot \Psi M_{j}=0 \forall i, j=1,2, \ldots, N \text { and } i \neq j
$$

Considering the objective of minimizing losses in a distribution system as a function of switch status $\left(\Psi M_{i}\right)$, it can be stated that:

Minimize: $f\left(\Psi M_{i}\right)$

Subject to the constraint for radial structure which is implemented through (4.12).

The problem in (4.12) and (4.13) has a discontinuous solution space as in (4.7) to (4.9). Just as the problem in (4.7) to (4.9) was recast in (4.10), the problem in (4.12) and (4.13) is recast as below in (4.14) whereby it becomes a continuous solution space which is differentiable and classical optimization technique can be used to find an optimal solution:

$$
\text { Minimize: } \quad f\left(\Psi M_{i}\right)+M \cdot \sum_{i} \sum_{j \neq i} \Psi M_{i} \cdot \Psi M_{j}
$$

Accordingly, in the next section the reconfiguration of radial distribution systems is formulated using complementarity constraints. 


\subsection{Proposed Problem Formulation}

\subsubsection{Loss Formula}

Consider a radial distribution system (RDS) with $N B$ buses. The objective is to minimize the total active-power loss. In the proposed algorithm, the loss is defined as follows:

$$
\text { Minimize: } P_{\text {Loss }}=\sum_{i=1}^{N B} P_{L}(V, \delta)
$$

where $\mathrm{P}_{\mathrm{L}}$ is the net active-power injected by the $i^{t h}$ bus into the connected lines and $\mathrm{V} \angle \delta$ is the vector of voltage phasors.

\subsubsection{Network Representation}

Let the RDS have $N L$ lines and $\left[Y_{L}\right]$ be a diagonal line admittance matrix of dimension $N L \cdot N L$. Let $\left[Y_{I}\right]$ be the bus incidence matrix. Let $[\Psi M]$ be a diagonal matrix with elements corresponding to switches where ones indicate open and zeros indicate otherwise. For the $i^{\text {th }}$ line segment, if there is a switch, $\Psi M_{i}$ is equal to one if the switch is open, and equal to zero if the switch is closed. Accordingly, the system admittance matrix in the bus frame of reference, the $Y$ - $B U S$, can be computed as below:

$$
\mathrm{Y}_{\mathrm{B}}=\left[\mathrm{Y}_{\mathrm{I}}\right] \cdot[\mathrm{I}-[\Psi \mathrm{M}]] \cdot\left[\mathrm{Y}_{\mathrm{L}}\right] \cdot\left[\mathrm{Y}_{\mathrm{I}}\right]^{\mathrm{T}}
$$

Using the Y-BUS matrix, Bus Power Balance equations may be computed as below and it has been illustrated in Fig. 2: 


$$
\mathrm{P}_{\mathrm{G}}-\mathrm{P}_{\mathrm{D}}-\mathrm{P}_{\mathrm{L}}\left(\mathrm{V}, \delta, \mathrm{Y}_{\mathrm{B}}\right)=0
$$

$$
\mathrm{Q}_{\mathrm{G}}-\mathrm{Q}_{\mathrm{D}}-\mathrm{Q}_{\mathrm{L}}\left(\mathrm{V}, \delta, \mathrm{Y}_{\mathrm{B}}\right)=0
$$

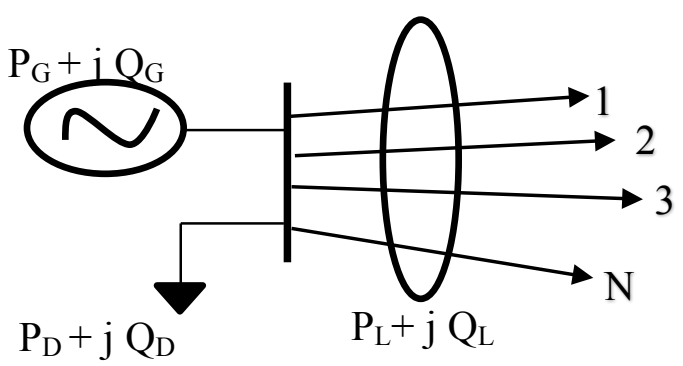

Fig. 4.2 $\mathrm{P}_{\mathrm{L}}$ and $\mathrm{Q}_{\mathrm{L}}$ are Sum of Power Flowing from the Bus into Connected Lines

where $\mathrm{P}_{\mathrm{G}}$ and $\mathrm{Q}_{\mathrm{G}}$ are vectors of bus-wise active and reactive power generations, $\mathrm{P}_{\mathrm{D}}$ and $\mathrm{Q}_{\mathrm{D}}$ are vectors of bus-wise active and reactive power loads and $\mathrm{P}_{\mathrm{L}}\left(\mathrm{V}, \delta, \mathrm{Y}_{\mathrm{B}}\right)$ and $\mathrm{Q}_{\mathrm{L}}\left(\mathrm{V}, \delta, \mathrm{Y}_{\mathrm{B}}\right)$ are vectors of active and reactive powers injected from buses into respective connected lines.

\subsubsection{Network Radial Structure - Complementarity Constraints}

Let a RDS have $N_{S}$ loops. Let $N_{S}(s)$ be a set of line indices comprising the $s^{\text {th }}$ loop. Writing complementarity constraint functions, one may write using (4.9) as below:

$$
\Psi M_{i} \cdot \Psi M_{j}=0 \forall i \neq j, i \in N_{S}(s), j \in N_{S}(s)
$$

The formulation (4.15) to (4.19) describes the optimal reconfiguration challenge which has a discontinuous solution space and cannot be solved using classical optimization techniques. Hence, 
we use complementarity technique to transform (4.15) to (4.19) in the following text such that it has a continuous solution space and therefore classical optimization techniques can be used.

\subsubsection{Complete Formulation}

The complete objective function for the optimization (4.20) is created as below using (4.15) to (4.19).

Minimize:

$$
f=\sum_{\mathrm{i}=1}^{\mathrm{NB}} \mathrm{P}_{\mathrm{L}}(\mathrm{V}, \delta)+\mathrm{M} \cdot \sum_{\mathrm{s}=1}^{\mathrm{NS}}\left[\sum_{\mathrm{i} \in \mathrm{NS}(\mathrm{s}), \mathrm{j} \in \mathrm{NS}(\mathrm{s}), \mathrm{i} \neq \mathrm{j}} \Psi \mathrm{M}_{\mathrm{i}} \cdot \Psi \mathrm{M}_{\mathrm{j}}\right]+\mathrm{M} \cdot \sum_{\mathrm{s}=1}^{\mathrm{NS}}\left[1-\sum_{\mathrm{i} \in \mathrm{NS}(\mathrm{s})} \Psi \mathrm{M}_{\mathrm{i}}\right]^{2}
$$

The first term in the objective function $\left(\sum_{\mathrm{i}=1}^{\mathrm{NB}} \mathrm{P}_{\mathrm{L}}(\mathrm{V}, \delta)\right)$ represents the active-power loss of the system which is meant to be minimized.

The second term $\left(\mathrm{M} \cdot \sum_{\mathrm{s}=1}^{\mathrm{NS}}\left[\sum_{\mathrm{i} \in \mathrm{NS}(\mathrm{s}), \mathrm{j} \in \mathrm{NS}(\mathrm{s}), \mathrm{i} \neq \mathrm{j}} \Psi \mathrm{M}_{\mathrm{j}} \cdot \Psi\right)\right.$, when minimized, ensures that the product of switch states equals zero in each loop which means only one switch per loop has a non-zero value.

The third term $\left(\mathrm{M} \cdot \sum_{\mathrm{s}=1}^{\mathrm{NS}}\left[1-\sum_{\mathrm{i} \in \mathrm{NS}(\mathrm{s})} \Psi \mathrm{M}_{\mathrm{i}}\right]^{2}\right)$, ensures that the only non-zero state of a switch in the loop equals to 1 , while the square avoids negative values.

In view of that, the global minimum of the second and third terms are both zero.

The objective (4.20) is subject to:

(1)Power Balance Equations: (4.17) and (4.18), 
(2) Voltage Limits:

$$
\underline{V} \leq V_{i t} \leq \bar{V}
$$

(3)Limits on $\Psi M$ :

$$
0 \leq \Psi M \leq 1
$$

This formulation can be readily solved using a classical nonlinear optimization solver.

\subsection{Results and Discussions}

The proposed methodology was tested on 7-bus, IEEE 33-bus and IEEE 69-bus systems. The 7-bus system is solved using both the proposed method, as well as GA method for direct comparison purposes. The IEEE 33-bus and IEEE 69-bus systems are solved using only the proposed method, and then the results are compared with various solution techniques available in the literature.

\subsubsection{The 7-bus System}

The system has only one loop as shown in Fig. 4.3 and the system data are given in Table 4.1.

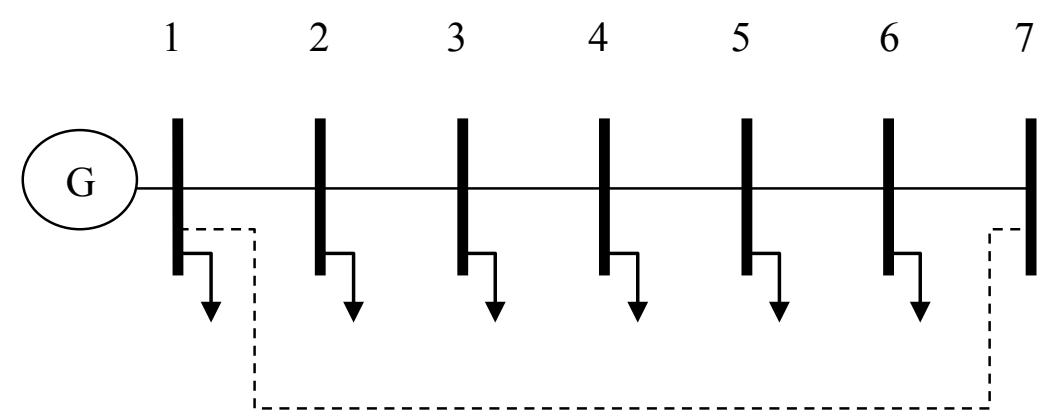

Fig. 4.3 Schematic of the 7-bus System 
Table 4.1 Data for 7-bus System (12.66 kV)

\begin{tabular}{c|c|c|c|c|c}
\hline Branch \# & From Bus & To Bus & $\begin{array}{c}\text { Resistance } \\
\text { (Per unit) }\end{array}$ & $\begin{array}{c}\text { Reactance } \\
\text { (Per unit) }\end{array}$ & $\begin{array}{c}\text { Load at To } \\
\text { Bus (MVA) }\end{array}$ \\
\hline 1 & 1 & 2 & 0.0922 & 0.0407 & $0.10+\mathrm{j} \mathrm{0.06}$ \\
\hline 2 & 2 & 3 & 0.4930 & 0.2510 & $0.09+\mathrm{j} 0.04$ \\
\hline 3 & 3 & 4 & 0.3660 & 0.1864 & $0.09+\mathrm{j} 0.04$ \\
\hline 4 & 4 & 5 & 0.3811 & 0.1941 & $0.12+\mathrm{j} 0.08$ \\
\hline 5 & 5 & 6 & 0.1872 & 0.6188 & $0.06+\mathrm{j} 0.03$ \\
\hline 6 & 6 & 7 & 1.7114 & 1.2351 & $0.06+\mathrm{j} 0.02$ \\
\hline 7 & 7 & 1 & 1.0300 & 0.7400 & 0 \\
\hline
\end{tabular}

\subsubsection{Result of Proposed Method Using Complementarity}

After optimization using (4.16), (4.17), (4.18), (4.20), (4.21) and (4.22) the optimal switch to open is determined to be at branch 5, which is located between buses 5 and 6. Fig. 4.4 shows the voltage profile before and after optimization. 


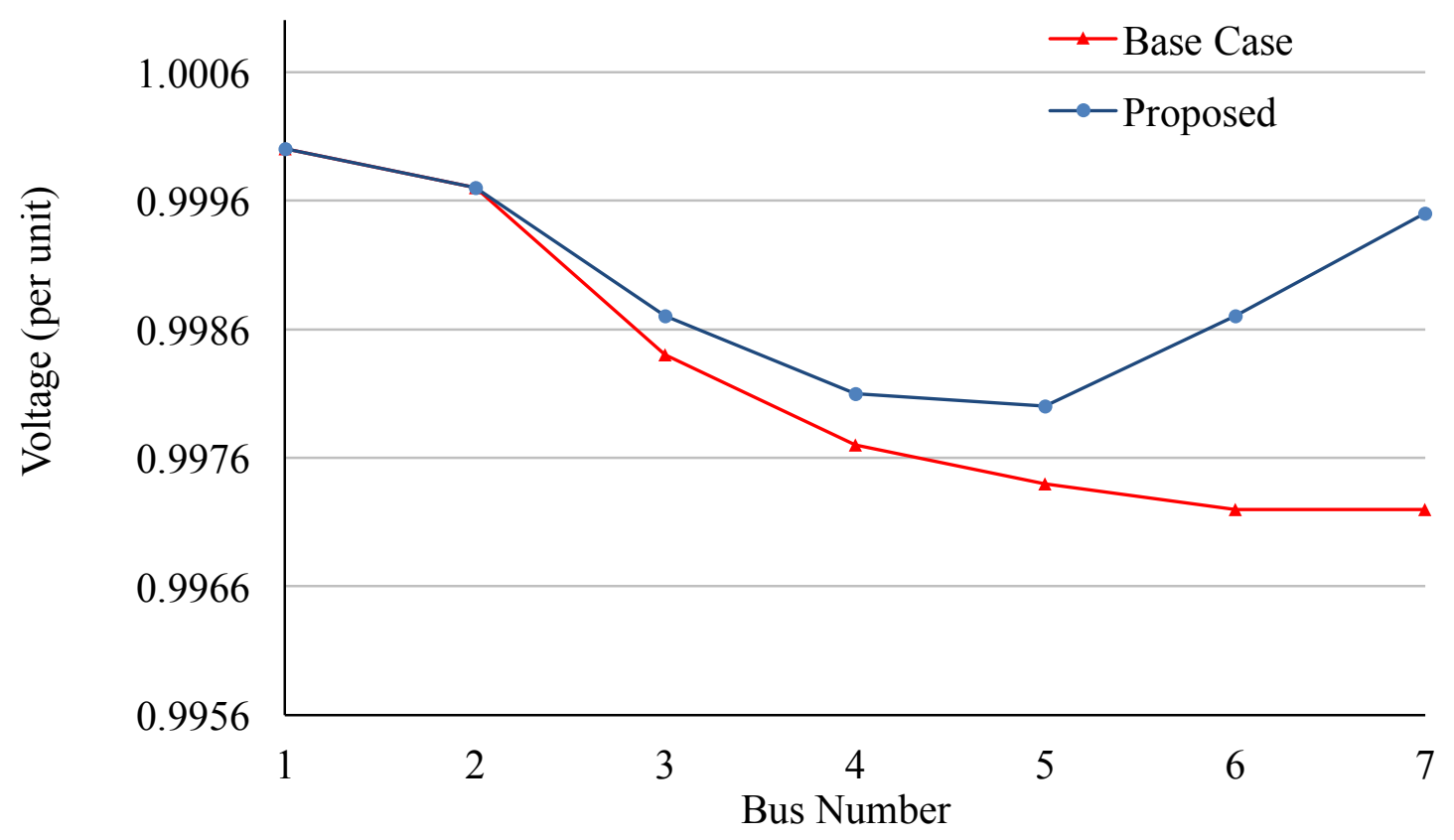

Fig. 4.4 Voltages of Each Individual Bus: 7-bus System

Fig. 4.5 shows the comparison of active-power flow of each branch before and after optimization. Before optimization, the tie-line 7 is open, so there is no power flowing into that branch. However, the result after optimization shows that there is no power flow in branch 5 since the switch is open at this branch. Also, it can be seen that the amount of flow has been decreased after optimization which is a sign of minimized loss. 


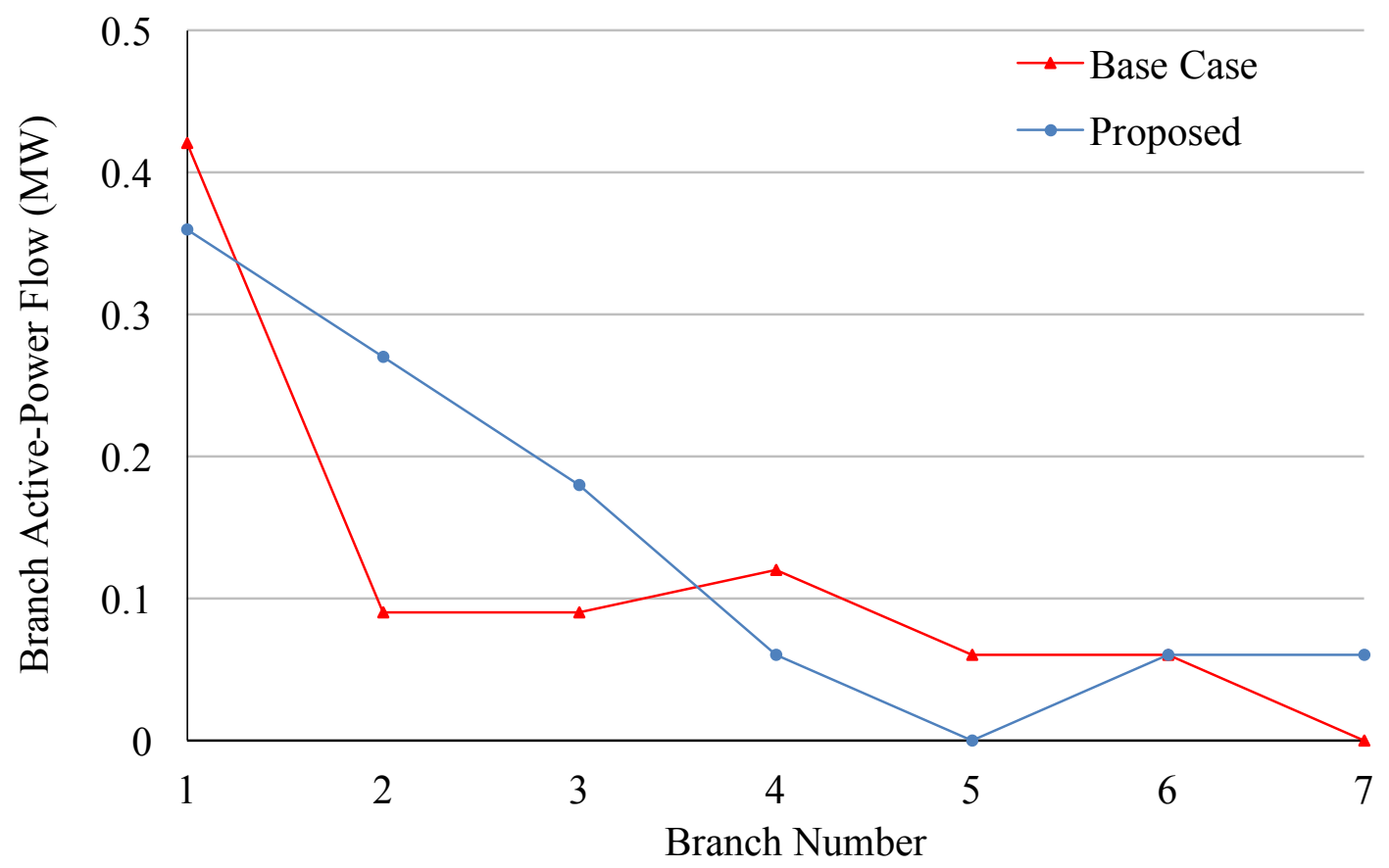

Fig. 4.5 Comparison of Active-Power Flow of Each Branch Before and After Optimization: 7-bus System

Table 4.2 compares the total system active-power loss before and after optimal reconfiguration using the proposed algorithm of nonlinear optimization technique with complementarity constraints.

Table 4.2 Comparison of Base Case and Optimal Solution

\begin{tabular}{c|c|c|c}
\hline Case & System Active-power Loss (kW) & $\begin{array}{c}\text { Worst Voltage } \\
\text { (per unit) }\end{array}$ & Open Branch \\
\hline Original & 0.77 & 0.9972 & Branch 7 \\
\hline Proposed & 0.57 & 0.9980 & Branch 5 \\
\hline
\end{tabular}




\subsubsection{The IEEE 33-bus System}

This distribution system has been widely used in research and literature for the purpose of reconfiguration [47]. The system has 5 loops. The number of switches in those 5 loops is respectively $6,4,5,4$ and 5 totaling to 24 switches. In each loop, only one switch must be open so that radial structure is preserved while the total active-power loss of the system is minimized. The active-power loss of the system before reconfiguration is $0.211 \mathrm{MW}$ and the minimum system voltage is 0.9038 per unit. Fig. 4.6 shows the schematic and the data can be found in Appendix B.
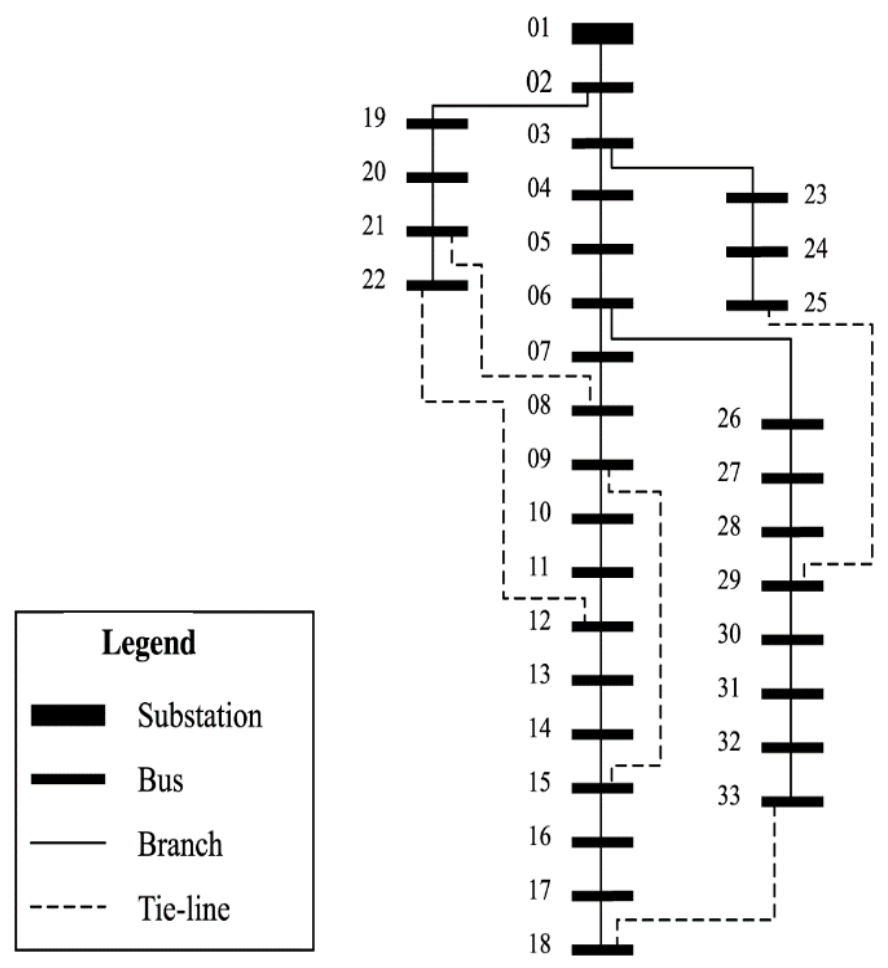

Fig. 4.6 Single Line Diagram of the 33-bus System

The dotted lines represent the open branches before optimization. The open branches in the basecase (before optimization) are: $33,34,35,36$ and 37 . After the system is optimized by the proposed 
complementarity algorithm, the best configuration determined open branches as: 7, 9, 14, 32 and 37 .

The bus voltages for this test system are limited between 0.9 to 1.05 per unit. A comparison between voltage profiles of base case and optimal solution is given in Fig. 4.7. The voltage profile after optimization is significantly improved. Also, Fig. 4.8 shows a comparison between the activepower flow of each branch for the base and optimized cases.

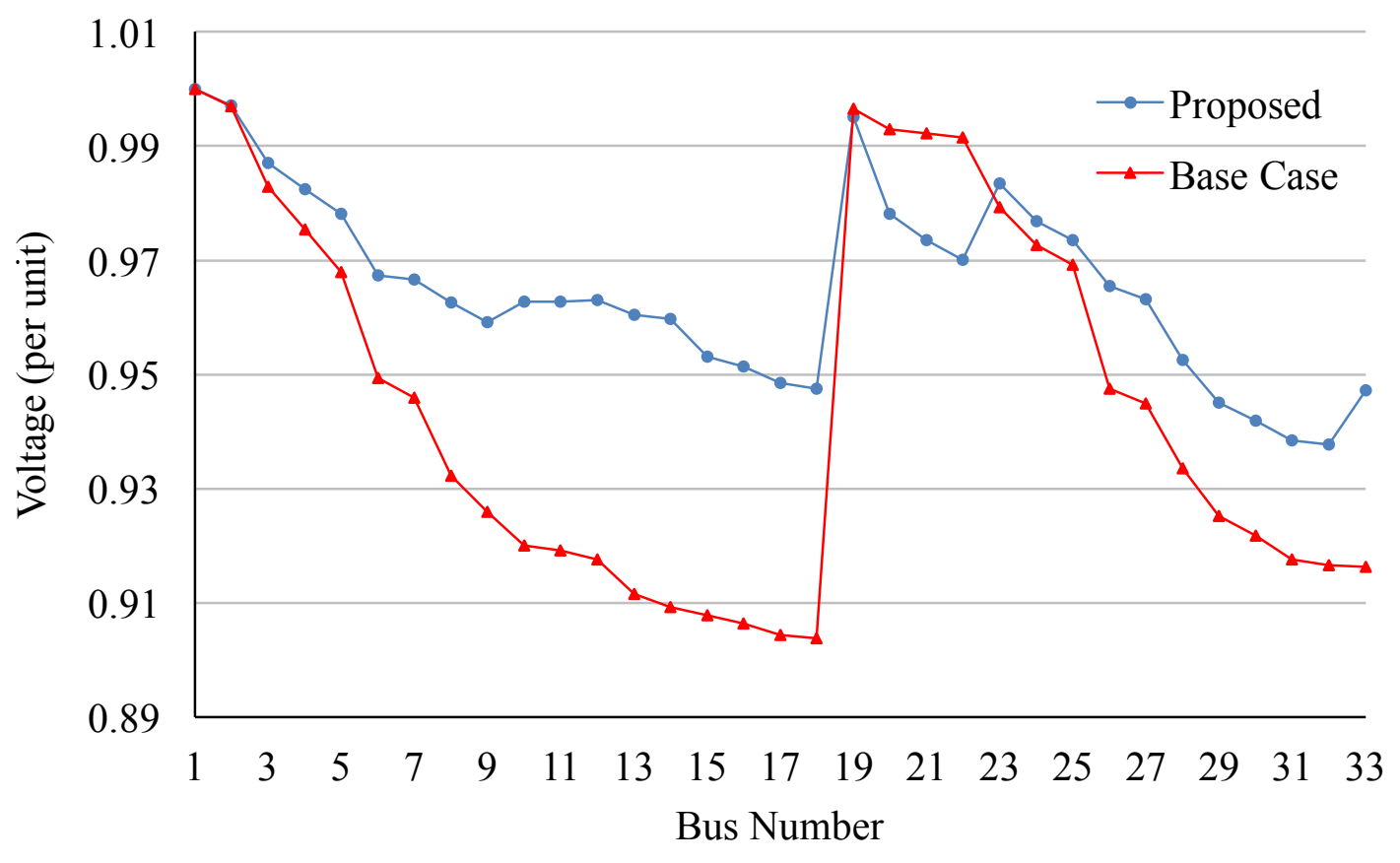

Fig. 4.7 Voltages of Each Individual Bus: IEEE 33-bus System 


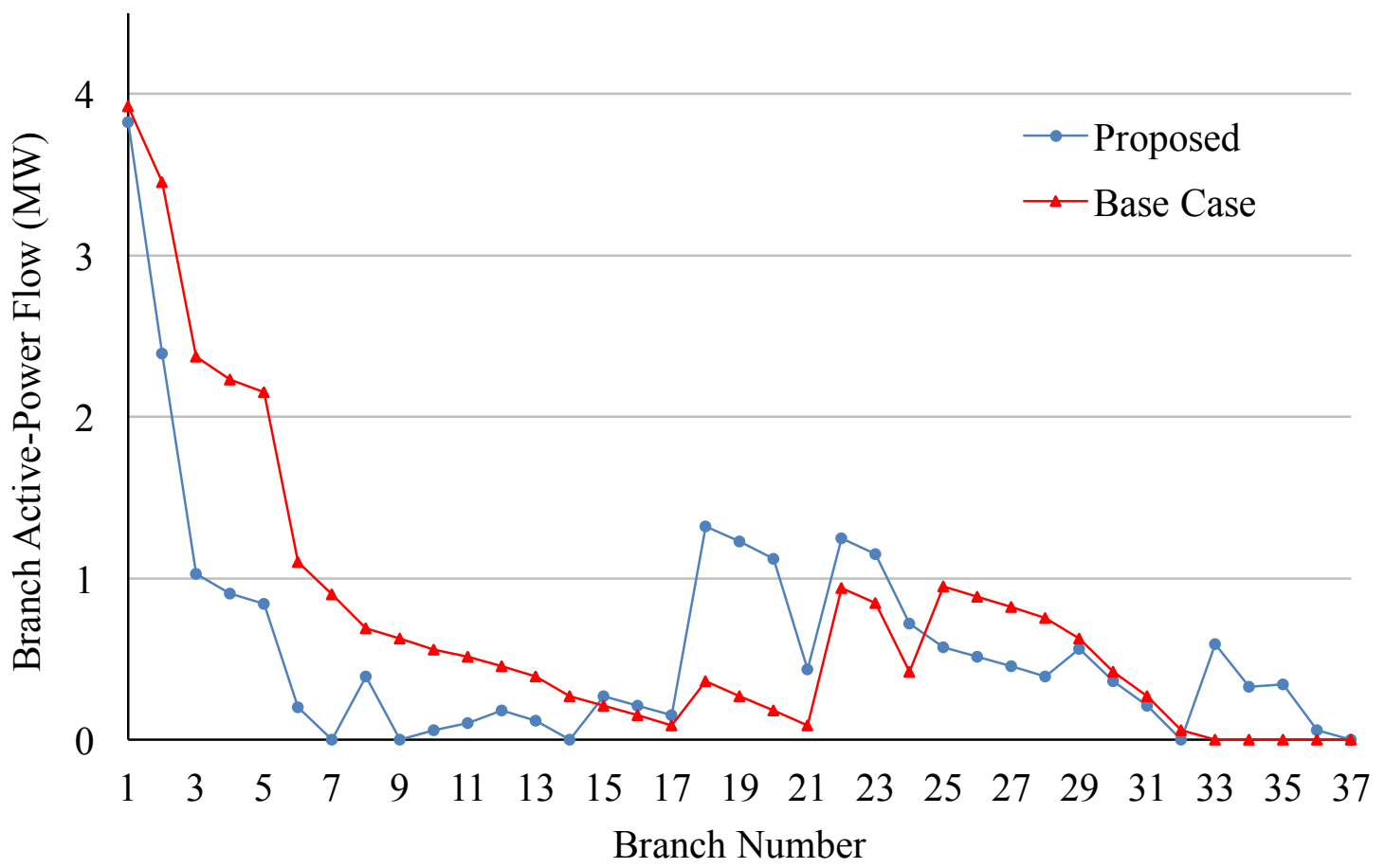

Fig. 4.8 Comparison of Active-Power Flow of Each Branch Before and After Optimization: IEEE

\section{3-bus System}

It is evident that the optimally reconfigured system has a better voltage solution and lighter loads flowing through lines.

Table 4.3 compares the running time and the total system active-power loss between the base case and optimal case. Also, in order to demonstrate performance of the proposed method, several other solutions published in literature have been compared in the table based on the same IEEE 33bus system. The worst voltage shows a big improvement compare to the base case and the voltage profile is also better than any other method shown in the table. 
Table 4.3 Comparison of Base Case and Optimal Solution for IEEE 33-bus System

\begin{tabular}{|c|c|c|c|c|c|c|}
\hline & \multirow{2}{*}{$\begin{array}{l}\text { Base } \\
\text { Case }\end{array}$} & \multicolumn{5}{|c|}{ Optimal Reconfiguration } \\
\hline & & HSA [47] & GA [56] & RGA [71] & ITS [72] & $\begin{array}{c}\text { Proposed } \\
\text { Method }\end{array}$ \\
\hline $\begin{array}{c}\text { Open } \\
\text { Branches }\end{array}$ & $\begin{array}{c}33,34, \\
35,36,37\end{array}$ & $\begin{array}{c}7,10,14,36 \\
37\end{array}$ & $\begin{array}{c}7,9,14,32,3 \\
7\end{array}$ & $\begin{array}{c}7,9,14,32 \\
37\end{array}$ & $\begin{array}{c}7,9,14,36 \\
37\end{array}$ & $7,9,14,32,37$ \\
\hline $\begin{array}{c}\text { Worst } \\
\text { Voltage (p.u.) }\end{array}$ & 0.9038 & 0.9342 & 0.929 & 0.931 & 0.921 & 0.9378 \\
\hline $\begin{array}{c}\text { Active-power } \\
\text { Loss } \\
\text { (MW) }\end{array}$ & 0.211 & $0.139-0.195$ & $\begin{array}{l}0.141- \\
0.202\end{array}$ & $\begin{array}{l}0.139- \\
0.198\end{array}$ & $\begin{array}{l}0.139- \\
0.196\end{array}$ & 0.139 \\
\hline $\begin{array}{c}\text { CPU } \\
\text { Time(s) }\end{array}$ & -- & 7.2 & 19.1 & 13.8 & 8.1 & 6.37 \\
\hline Runs & -- & 200 & 200 & 200 & 200 & 1 \\
\hline
\end{tabular}

The penalty factor "M" used for this system was 1000 .

In addition, It should be noted that GA and other heuristic optimization methods may not yield the optimal solution consistently, and they normally require several trial runs before an optimal solution is reached. Hence the CPU time of 7.2 seconds from the Harmony Search Algorithm [47] is not consistent and is achieved by solving the problem 200 times. However it can be seen from the above table that the computation time of the proposed method is always 6.37 seconds which is faster than any other method available in literature. This new method makes it possible to reach the optimum result in only one try. The proposed solution method is suitable for real-time application with smart radial distribution system and it becomes more obvious when we compare the complete time 
required to solve.

\subsubsection{The IEEE 69-bus System}

The single line diagram is shown in Fig. 4.9 and the data of the system is published in [61] and available in Appendix B. The system has 5 tie-switches located at branches 69, 70, 71, 72 and 73.

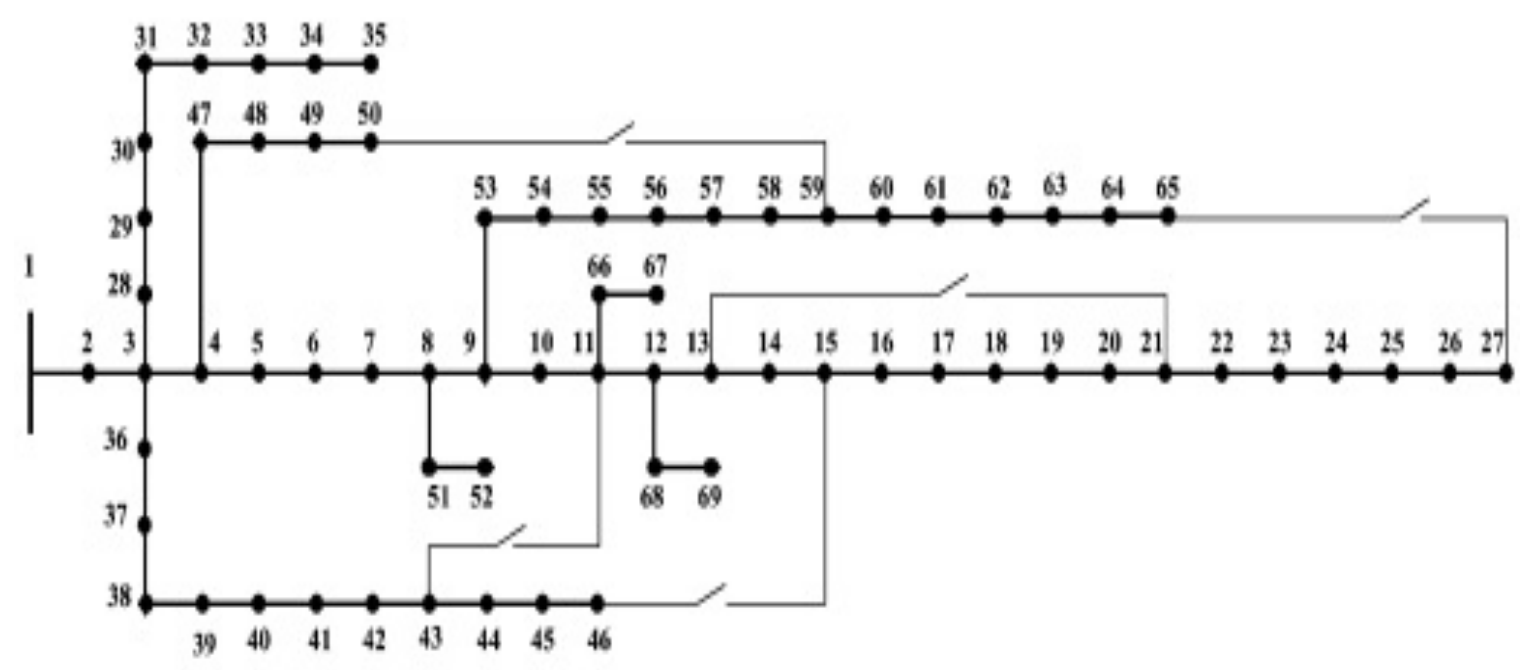

Fig. 4.9 Single Line Diagram of The IEEE 69-bus System

The active-power loss of the system before reconfiguration is $0.22495 \mathrm{MW}$ and the minimum system voltage is 0.9092 per unit. After optimally reconfiguring this system using the proposed method, the total active-power loss is minimized and the voltage profile is improved. The optimal solution is provided in Table 4.4 .

The voltage profiles of the base case and optimized solution are portrayed in Fig. 4.10. The worst voltage after optimization is 0.95 .

Similarly, Fig. 4.11 shows branch-wise active-power flow solutions in MW, before and after optimization. Comparing the base-case and optimal solution, it is clear that the optimized solution has been much improved. The result is compared to the Fuzzy Multi-Objective Optimization [61] 
and Particle Swarm Optimization Algorithm [73] in Table 4.4. While the proposed method has found a better solution with regards to active-power loss and minimum bus voltage, it only took 33.9 seconds and one execution for it to reach the optimum result. On the other hand, it takes 800 seconds for PSO in [73] to obtain its best results.

Table 4.4 Comparison of Base Case and Optimal Solution for IEEE 69-bus System

\begin{tabular}{|c|c|c|c|c|}
\hline & \multirow{2}{*}{ Base Case } & \multicolumn{3}{|c|}{ Optimal Reconfiguration } \\
\hline & & Fuzzy MO [61] & PSO [73] & Proposed Method \\
\hline $\begin{array}{c}\text { Open } \\
\text { Branches }\end{array}$ & $\begin{array}{c}69,70,71, \\
72,73\end{array}$ & $56,70,63,69,14$ & $59,71,62,70,15$ & $57,70,61,69,12$ \\
\hline $\begin{array}{l}\text { Worst } \\
\text { Voltage } \\
\text { (p.u.) }\end{array}$ & 0.9092 & 0.9483 & 0.94247 & 0.95 \\
\hline $\begin{array}{c}\text { Active-power } \\
\text { Loss } \\
\text { (MW) }\end{array}$ & 0.22495 & 0.0996 & 0.099635 & 0.09877 \\
\hline $\begin{array}{c}\text { CPU } \\
\text { Time(s) }\end{array}$ & -- & Not Available & 8 & 33.9 \\
\hline Runs & - & 200 & 100 & 1 \\
\hline
\end{tabular}




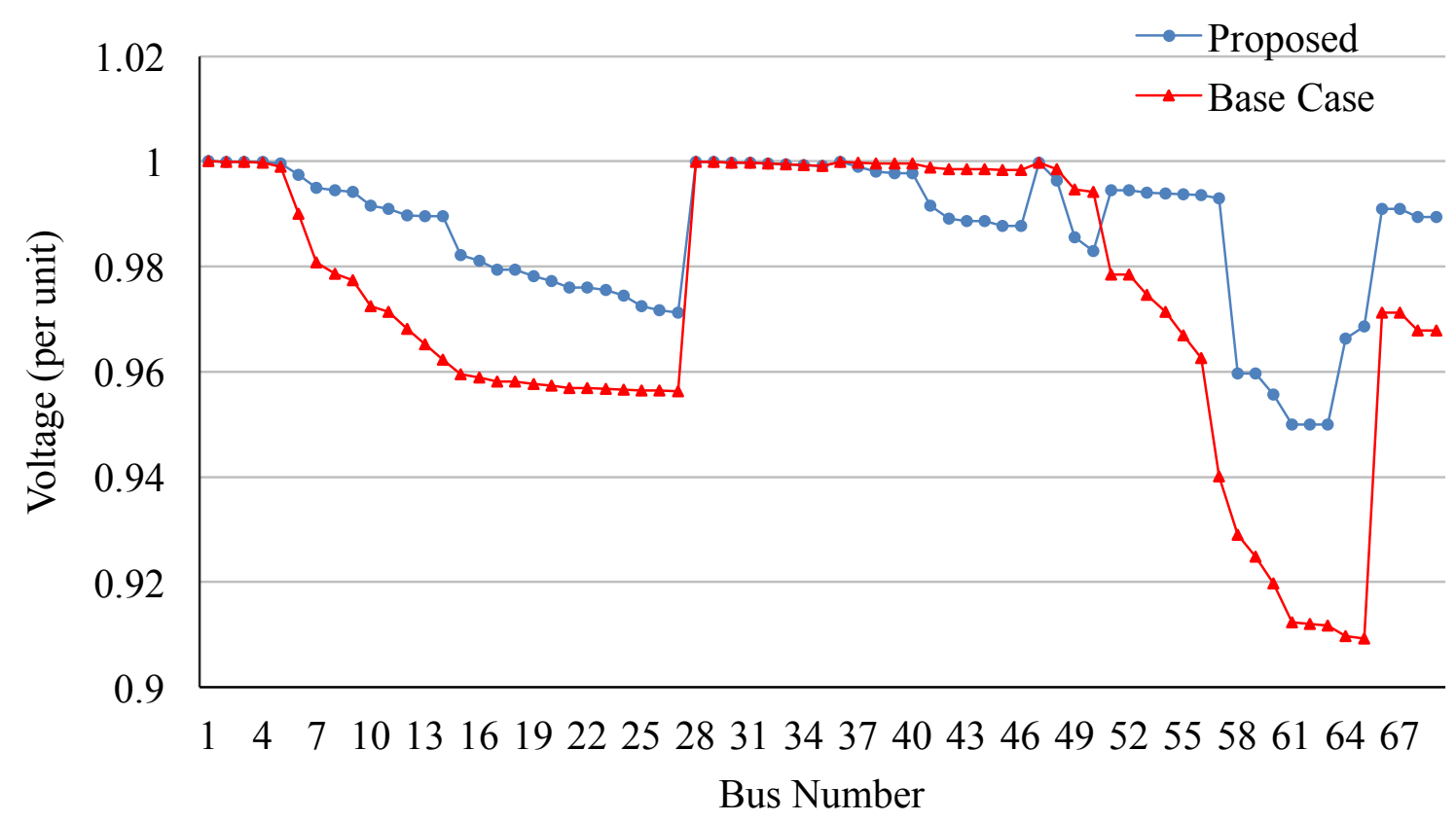

Fig. 4.10 Voltages of Each Individual Bus: IEEE 69-bus System

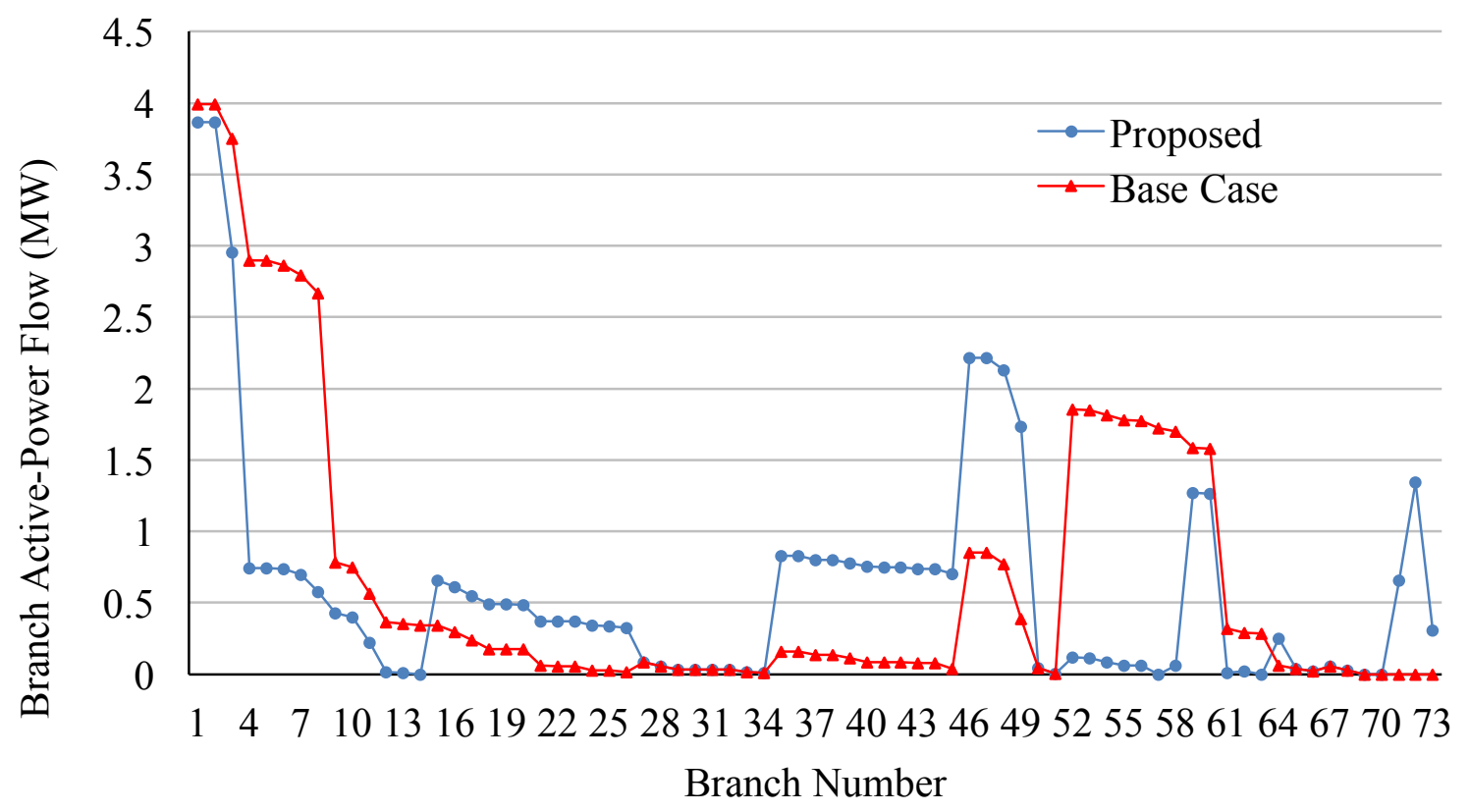

Fig. 4.11 Comparison of Active-Power Flow of Each Branch Before and After Optimization: IEEE 69-bus System 
The proposed method changes the way network reconfiguration was approached hitherto. Literature shows that heuristic methods are predominantly used which renders the solution methods unsuitable for real-time use. With the advent of smart grid technologies, enabling real-time management of smart radial distribution systems via DMS (distribution management systems) using real-time optimal reconfiguration type algorithms can bring benefits such as improved reliability, performance and efficiency.

To enable real-time performance, a complementarity technique is used to transform the mixed integer nonlinear optimization challenge of optimal reconfiguration with discontinuous solution space into a problem with a continuous solution space. This transformation allows solution(s) via an ordinary nonlinear programming technique. In addition, benefits are:

-Single shot solution.

-Fast solution in seconds for real-time use.

-Use of a classical nonlinear optimization technique.

-Easy extension to an optimal power flow type algorithm.

\subsection{Chapter Summary}

In this work, the discontinuous solution space is transformed into a continuous one using complementarity technique whereby conventional NLP optimization techniques can be used making the method suitable for real-time applications. The proposed method opens doors to the use of other methods and concepts of optimal power flow such as nodal pricing being applied to RDS.

The proposed method is demonstrated on standard IEEE test systems where it produces the same or even better results, but in much shorter time than that required by heuristic methods. Optimal solutions obtained in 33-bus and 69-bus systems were compared with published results and shown 
to be comparable or better in accuracy, time and robustness.

One interesting comparison could be held between deterministic and heuristic optimization routines when solving the problem of distribution system reconfiguration. Deterministic methods are known for lack of ability to converge to global minima all the time. However, convergence to the suboptimal solution obtained by these methods is much faster and much more robust than heuristics techniques. On the other hand, although heuristic algorithms are capable of reaching a global minimum solution, the convergence time is much longer and nearly uncertain. Due to the stochastic nature of heuristic algorithms, indefinite numerical experimentation is typically required before a satisfactory solution is obtained. Therefore, heuristic optimization is unsuitable for online and real-time solutions of the present problem because of such drawbacks. 


\section{CHAPTER 5}

\section{CONCLUSION AND FUTURE WORK}

General conclusions and the key contributions of this dissertation work are summarized in this chapter. Recommended future research in power system optimization studies as it relates to this dissertation is also discussed. The book [73] was immensely valuable in learning the fundamentals of power system optimization and acquire the knowledge of different techniques of optimization.

\subsection{Conclusion and Major Contributions}

The following targets have been achieved and are presented below as key research contributions:

1) A new FIM model for the DSA planning process was proposed, which includes the possibility of installing BESS as a solution to integrate new loads and generators. Based on the analyses presented here, one can see that the proposed model is flexible, automatically selecting the installation of new feeders and/or BESS while optimally sizing the BESS. In addition, the results show that in the presence of high penetration of PV systems and large differences between the average power and the peak power, there is a higher arbitrage opportunity for BESS, mainly in costly underground systems. For a 3-Bus simplified Canadian system, it was shown in Table 2.12 that the BESS with the 5.22 MW and 22.98MWh size is a more economical choice than using an additional feeder. Also, using the proposed algorithm on the 33-Bus system resulted in choosing a BESS with the optimized size shown in Table 2.18 as the more cost-effective option. A planning with BESS costs $\$ 36,509,475$ 
million dollars while planning with an additional feeder would cost $\$ 37,439,588$ million dollars. From these results, it is clear that the proposed DSA planning method is able to include BESS units and additional feeder circuits into the asset planning solution. The proposed method considers the myriad of options to select the least costly annual asset plan. It compares the options evenly based on an evolution of the total annual asset costs for delivering energy to the connected customers.

2) Based on the created FIM model, the proposed method is a multi-objective algorithm with contradicting objectives of SAIDI minimization and total cost minimization. Fuzzy optimization is used to handle multiple objectives that cannot be combined into a single objective. This method has been used due to the lack of certainty in how to calculate customer interruption cost in literature. Most of the research in this area is not practical, as unreasonable values of customer interruption costs are used for translating SAIDI values in minutes per year to dollars per year. While the proposed algorithm minimizes SAIDI for the upgraded system, it does not depend on the customer interruption cost for optimization. Therefore, the results are more reliable compared to the existing theories and models. Testing the following method on the 33-Bus system shows that the BESS is the cheaper option compare to additional feeders. The optimal size of the BESS unit as given in Table 3.5 is $1.45 \mathrm{MW}$ and $11.04 \mathrm{MWh}$, while the total planning cost of using BESS is $\$ 178,700$ compare to $\$ 902,009$ when using an extra feeder. Also, the proposed method was compared to recently published method in literature in Table 3.8 which shows that the proposed method is superior.

3) To enable real-time performance in operating distribution systems, a complementarity technique is used to transform the mixed integer nonlinear optimization challenge of optimal reconfiguration with discontinuous solution space into a problem with a continuous solution space. This transformation allows solution(s) via an ordinary nonlinear programming technique. Table 4.2 
shows that the complementarity method works on a simple 7-Bus system. Then for more complicated systems, the proposed method was tested on 33-Bus and 69-Bus system which had more loops and switches. For 33-Bus system, Table 4.4 shows that the proposed method needs only 6.37 seconds and a single run to achieve the optimum result, while the closest method in literature needs at least 7.2 seconds for each run and 200 runs which is equivalent of 1,440 seconds. Also, for the 69-Bus system, Table 4.5 indicates that the proposed algorithm needs 33.9 seconds and a single run to reach the optimum point, while the available methods in literature need 8 seconds for each run and a minimum of 100 runs, which is equal to 800 seconds. Although heuristic algorithms in literature can reach a global minimum solution, the convergence time is much longer and very uncertain. Due to the stochastic nature of heuristic algorithms, indefinite numerical experimentation is typically required before a satisfactory solution is obtained. On the other hand, the proposed method produces the same or even better results than heuristic methods in a much shorter time using a single run.

Overall, these algorithms and methods are very practical for real market applications. Many electrical distribution companies, such as Toronto Hydro-Electric System Limited (THESL) in Ontario, are seeking new methods to manage planning and operating of their assets. The proposed techniques are a big step towards that path. 


\subsection{Recommendations}

A number of future directions for related research activities could be explored:

1) Linearization of the formulation which needs extensive math work, would give the algorithms in Chapter 2 and 3 a direct proof and also make them significantly faster.

2) The complementarity method can be applied to the planning algorithms and FIM.

3) The possibility of mixing different energy storage devices in the grid, for example using a flywheel in conjunction with lithium-ion battery. These devices have different life time and maintenance characteristics that may optimize the total cost further when used together. 


\title{
APPENDiX A
}

\section{FUNDAMENTALS AND TECHNIQUES}

\author{
A.1 Power Balance Equations \\ DISTRIBUTION NETWORK CONSTRAINTS MUST BE ENFORCED IN THE PLANNING AND \\ OPERATING PROBLEMS TO PROVIDE A NETWORK FEASIBLE SOLUTION. POWER \\ BALANCE EQUATIONS, AS DISCUSSED BELOW, ARE THE BASIS FOR \\ DEVELOPING THE CONSTRAINTS.
}

FIG. A.1 SHOWS THE NET POWER INJECTION OF A PRACTICAL BUS IN A POWER DISTRIBUTION SYSTEM. THE NET POWER INJECTION FROM THE BUS INTO THE CONNECTED LINES, WHICH IS THE FUNCTION OF BUS VOLTAGE PHASE ANGLES AND MAGNITUDES, MUST BE EQUAL TO TOTAL POWER GENERATION SUBTRACTED FROM THE TOTAL DEMAND ON THE BUS. TO SOLVE THESE BALANCE EQUATIONS, REACTIVE POWER GENERATIONS, LINE POWER FLOWS, AND BUS VOLTAGE PHASE ANGLES AND MAGNITUDES CAN BE CALCULATED.

$$
P L_{i}(V, \delta)=V_{i} \sum_{j}^{N B} V_{j}\left|Y_{i j}\right| \cdot \cos \left(\delta_{i}-\delta_{j}-\theta_{i j}\right) \forall i
$$




$$
Q L_{i}(V, \delta)=V_{i} \sum_{j}^{N B} V_{j} \cdot Y_{i j} \mid \cdot \sin \left(\delta_{i}-\delta_{j}-\theta_{i j}\right) \forall i
$$

In equations (A.1) and (A.2), $V$ is voltage magnitude of the $\mathrm{i}^{\text {th }}$ bus, and $\delta$ is the corresponding voltage phase angle. $Y_{i j}$ is an element of the bus admittance matrix $Y$, and $\theta_{i j}$ is the phase angle of $Y_{i j}$. After solving the power balance equations, bus voltage and reactive power generation can be obtained. $S L_{i}$ is the apparent power injected from the buses into the connected lines.

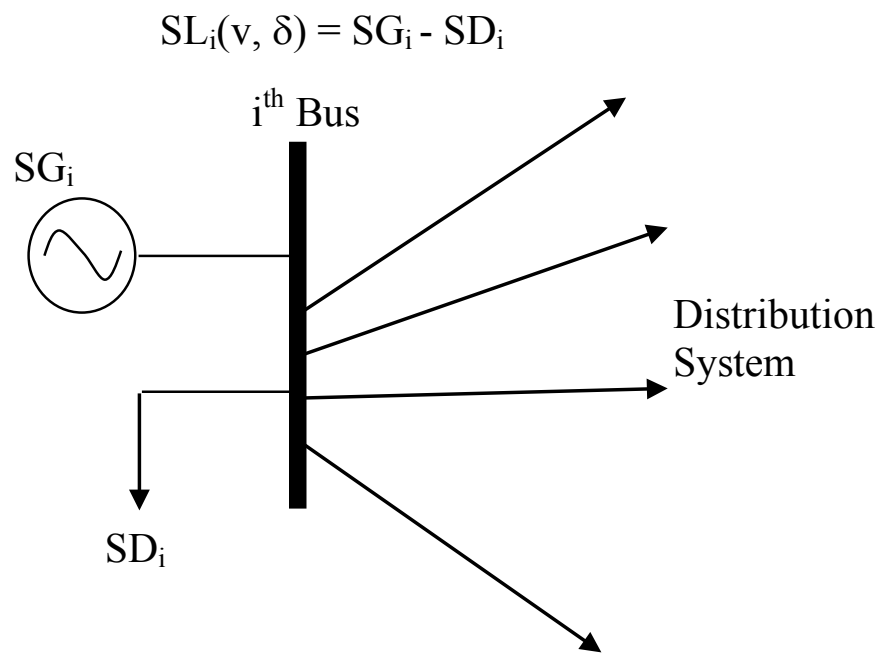

Fig. A.1 Net Power Injected into The Distribution System by the ith Bus

THESE POWER BALANCE EQUATIONS ARE CALLED AC POWER BALANCE EQUATIONS AND CONSIDER BOTH REAL AND REACTIVE POWERS. THESE POWER BALANCE EQUATIONS CAN BE SOLVED BY USING LOAD FLOW METHODS. 


\section{A.2 Derivation of AnNuity Formula With Interest Rate (PENAlty}

\section{FACTOR)}

The Present Worth $(P W)$ of a COnstant Annuity $(A N)$, FOR $N Y$ Periods is:

$$
\mathrm{PW}=\frac{A N}{(1+r)}+\frac{A N}{(1+r)^{2}}+\cdots+\frac{A N}{(1+r)^{N Y}}
$$

The discount factor can be defined as:

$$
\mathrm{a}=\frac{1}{(1+r)}
$$

substituting it into the (A.3):

$$
P W=A N \cdot\left(a+a^{2}+a^{3}+\cdots+a^{N Y}\right)
$$

To simplify this formula, $a^{N Y+1}, a^{N Y+2}$ and so on will be added to (A.5), and then subtract all the added terms:

$$
\left(\mathrm{a}+\mathrm{a}^{2}+\mathrm{a}^{3}+\cdots+\mathrm{a}^{N Y}\right)+\left(\mathrm{a}^{N Y+1}+\mathrm{a}^{N Y+2}+\cdots\right)-\left(\mathrm{a}^{N Y+1}+\mathrm{a}^{N Y+2}+\cdots\right)
$$

The (A.6) can be rewritten as:

$$
a\left(1+a+a^{2}+a^{3}+\cdots\right)-a^{N Y+1}\left(1+a+a^{2}+a^{3}+\cdots\right)
$$


Note that the infinite number of terms in each of the brackets is the same. To Simplify, a Random Number $(\mathrm{RN})$ is used:

$$
R N=\left(1+\mathrm{a}+\mathrm{a}^{2}+\mathrm{a}^{3}+\cdots\right)
$$

Now, observe that $R N=1+a \cdot R N$, which mean that:

$$
R N=\frac{1}{1-a}
$$

Therefore, the expression

$$
\left(1+\mathrm{a}+\mathrm{a}^{2}+\mathrm{a}^{3}+\cdots+\mathrm{a}^{N Y}\right)=a \cdot R N-a^{N Y+1} \cdot R N=\frac{a}{1-a}-\frac{a^{N Y+1}}{1-a}
$$

Now, replace $a$ with the discount factor $1 /(1+r)$ and simplify to get:

$$
\text { a. } R N-a^{N Y+1} \cdot R N=\frac{1}{r}\left(1-\frac{1}{(1+r)^{N Y}}\right)
$$

By replacing the expression in the bracket in (A.5) with what was achieved in (A.11), the following equation is created:

$$
P W=\frac{A N}{r}\left(1-\frac{1}{(1+r)^{N Y}}\right)
$$

which is the annuity formula.

$$
\text { Annuity: }\left(\frac{\mathrm{AN}}{\mathrm{PW}}, \mathrm{r} \%, \mathrm{NY}\right)=\mathrm{PW} \cdot \frac{\mathrm{r}(1+\mathrm{r})^{\mathrm{NY}}}{(1+\mathrm{r})^{\mathrm{NY}}-1}=\mathrm{AN}
$$




\section{A.3 SAIDI AND CUSTOMER INTERRUPTION COST DERIVATION}

It is known historically that the reliability of residential branches is not highly sensitive to loading. This is because the branch in only heavily loaded for a few hours per year. The situation is different for commercial/industrial (C\&I) feeders. However, with the emergence of Electric Vehicles, right now some of the residential branches are heavily loaded every day. It can be seen in Fig. A.2 that branches loaded at $90 \%$ of rating have an SAIDI of 1.4 hours, while feeders loaded at $100 \%$ of rating have an SAIDI of 1.9 hours.

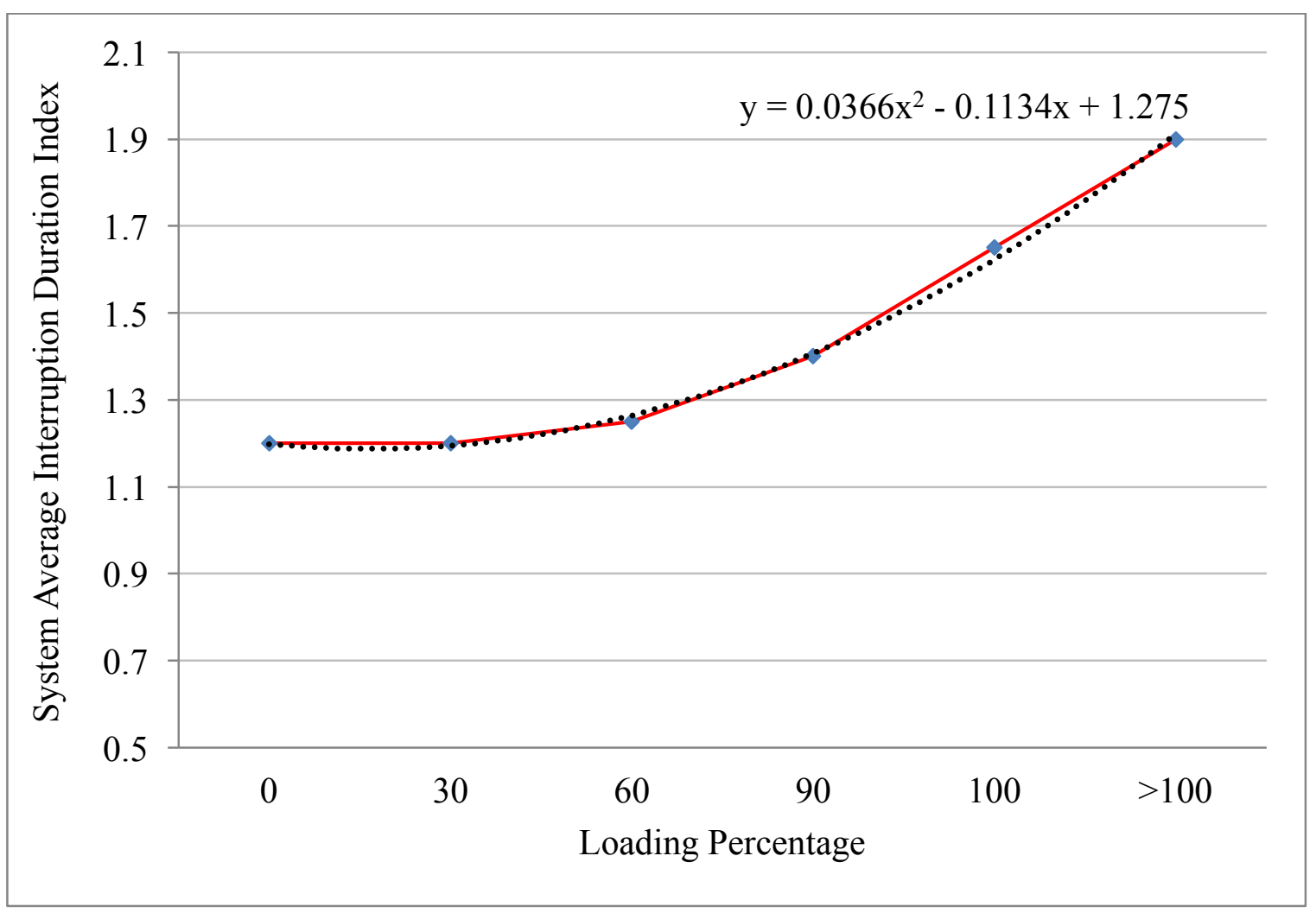


Fig. A.2 Variation of SAIDI (hour/year) versus Branch Loading Percentage $\frac{\overline{S F}}{S F E}$

Based on the trend line of the figure above, and knowing that $(\mathrm{y}=\mathrm{SAIDI})$ and $\left(\mathrm{x}=\frac{\overline{S F}}{S F E}\right)$, it can be concluded that:

$\mathrm{A}=0.0366$

$\mathrm{B}=-0.1134$,

$\mathrm{C}=1.275$

Therefore, the equation (2.8) becomes as follows for each circuit at each specific time zone:

$\operatorname{SAIDI}(S F E, \overline{S F})=\frac{A\left(\frac{\max _{t}\left\{S F_{l t}\right\}}{N C_{l} . S F E_{l}}\right)^{2}+B\left(\frac{t}{N C_{l} \cdot S F E_{l}}\right)+C}{N C_{l}}$

\section{APPENDIX B}

\section{DAta of Test Systems}

\section{B.1 IEEE 33-bus System}

The detailed transmission system data can be found in Table B.1, B.2 and B.3. The price data of CEG and WEG data are listed in the following tables. The system is tested for the OPF algorithm in Chapter 3. 
Table B.1 Data for IEEE 33-bus System

\begin{tabular}{c|c|c|c|c|c|c}
\hline Branch \# & $\begin{array}{c}\text { From } \\
\text { Bus }\end{array}$ & To Bus & $\begin{array}{c}\text { Resistance } \\
\text { (per unit) }\end{array}$ & $\begin{array}{c}\text { Reactance } \\
\text { (per unit) }\end{array}$ & $\begin{array}{c}\text { Load at To Bus } \\
\text { (MW) }\end{array}$ & $\begin{array}{c}\text { Load at To Bus } \\
\text { (MVAr) }\end{array}$ \\
\hline 1 & 1 & 2 & 0.0922 & 0.047 & 0.1 & 0.06 \\
\hline 2 & 2 & 3 & 0.493 & 0.2511 & 0.09 & 0.08 \\
\hline 3 & 3 & 4 & 0.366 & 0.1864 & 0.12 & 0.03 \\
\hline 4 & 4 & 5 & 0.3811 & 0.1941 & 0.06 & 0.02 \\
\hline 5 & 5 & 6 & 0.819 & 0.707 & 0.06 & 0.1 \\
\hline 6 & 6 & 7 & 0.1872 & 0.6188 & 0.2 & \\
\hline
\end{tabular}




\begin{tabular}{|c|c|c|c|c|c|c|}
\hline Branch \# & $\begin{array}{l}\text { From } \\
\text { Bus }\end{array}$ & To Bus & $\begin{array}{l}\text { Resistance } \\
\text { (per unit) }\end{array}$ & $\begin{array}{l}\text { Reactance } \\
\text { (per unit) }\end{array}$ & $\begin{array}{c}\text { Load at To Bus } \\
(\mathrm{MW})\end{array}$ & $\begin{array}{c}\text { Load at To Bus } \\
\text { (MVAr) }\end{array}$ \\
\hline 7 & 7 & 8 & 1.7114 & 1.2351 & 0.2 & 0.1 \\
\hline 8 & 8 & 9 & 1.03 & 0.74 & 0.06 & 0.02 \\
\hline 9 & 9 & 10 & 1.044 & 0.74 & 0.06 & 0.02 \\
\hline 10 & 10 & 11 & 0.1966 & 0.065 & 0.045 & 0.03 \\
\hline 11 & 11 & 12 & 0.3744 & 0.1238 & 0.06 & 0.035 \\
\hline 12 & 12 & 13 & 1.468 & 1.155 & 0.06 & 0.035 \\
\hline 13 & 13 & 14 & 0.5416 & 0.7129 & 0.12 & 0.08 \\
\hline 34 & 9 & 15 & 2 & 2 & 0.06 & 0.01 \\
\hline 15 & 15 & 16 & 0.7463 & 0.545 & 0.06 & 0.02 \\
\hline 16 & 16 & 17 & 1.289 & 1.721 & 0.06 & 0.02 \\
\hline 17 & 17 & 18 & 0.732 & 0.574 & 0.09 & 0.04 \\
\hline 18 & 2 & 19 & 0.164 & 0.1565 & 0.09 & 0.04 \\
\hline 19 & 19 & 20 & 1.5042 & 1.3554 & 0.09 & 0.04 \\
\hline 33 & 8 & 21 & 2 & 2 & 0.09 & 0.04 \\
\hline 21 & 21 & 22 & 0.7089 & 0.9373 & 0.09 & 0.04 \\
\hline 22 & 3 & 23 & 0.4512 & 0.3083 & 0.09 & 0.05 \\
\hline 23 & 23 & 24 & 0.898 & 0.7091 & 0.42 & 0.2 \\
\hline 24 & 24 & 25 & 0.896 & 0.7011 & 0.42 & 0.2 \\
\hline 25 & 6 & 26 & 0.203 & 0.1034 & 0.06 & 0.025 \\
\hline 26 & 26 & 27 & 0.2842 & 0.1447 & 0.06 & 0.025 \\
\hline 27 & 27 & 28 & 1.059 & 0.9337 & 0.06 & 0.02 \\
\hline
\end{tabular}




\begin{tabular}{c|c|c|c|c|c|c}
\hline Branch \# & From & To Bus & Resistance & Reactance & Load at To Bus & Load at To Bus \\
& Bus & & (per unit) & (per unit) & (MW) & (MVAr) \\
\hline 37 & 25 & 29 & 0.5 & 0.5 & 0.12 & 0.07 \\
\hline 29 & 29 & 30 & 0.5075 & 0.2585 & 0.15 & 0.1 \\
\hline 30 & 30 & 31 & 0.9744 & 0.963 & 0.21 & 0.04 \\
\hline 31 & 31 & 32 & 0.3105 & 0.3619 & - & - \\
\hline 35 & 22 & 12 & 2 & 0.5302 & - & - \\
\hline 14 & 14 & 15 & 0.591 & 0.526 & - & - \\
\hline 20 & 20 & 21 & 0.4095 & 0.4784 & & - \\
\hline 28 & 28 & 29 & 0.8042 & 0.7006 & & - \\
\hline 36 & 18 & 33 & 0.5 & 0.5 & & - \\
\hline
\end{tabular}

\section{B.2 IEEE 69-bus System}

Table B. 2 shows the bus data and Table B. 3 shows the branch data.

Table B.2 Bus Data for IEEE 69-bus System

\begin{tabular}{c|c|c}
\hline Bus Number & Load (MW) & Load (MVAr) \\
\hline 1 & 0 & 0 \\
\hline 2 & 0 & 0 \\
\hline 3 & 0 & 0 \\
\hline
\end{tabular}




\begin{tabular}{|c|c|c|}
\hline Bus Number & Load (MW) & Load (MVAr) \\
\hline 4 & 0 & 0 \\
\hline 5 & 0 & 0 \\
\hline 6 & 0.0026 & 0.0022 \\
\hline 7 & 0.0404 & 0.03 \\
\hline 8 & 0.075 & 0.054 \\
\hline 9 & 0.03 & 0.022 \\
\hline 10 & 0.028 & 0.019 \\
\hline 11 & 0.145 & 0.104 \\
\hline 12 & 0.145 & 0.104 \\
\hline 13 & 0.008 & 0.005 \\
\hline 14 & 0.008 & 0.0055 \\
\hline 15 & 0 & 0 \\
\hline 16 & 0.0455 & 0.03 \\
\hline 17 & 0.06 & 0.035 \\
\hline 18 & 0.06 & 0.035 \\
\hline 19 & 0 & 0 \\
\hline 20 & 0.001 & 0.0006 \\
\hline 21 & 0.114 & 0.081 \\
\hline 22 & 0.005 & 0.0035 \\
\hline 23 & 0 & 0 \\
\hline 24 & 0.028 & 0.02 \\
\hline 25 & 0 & 0 \\
\hline
\end{tabular}




\begin{tabular}{|c|c|c|}
\hline Bus Number & Load (MW) & Load (MVAr) \\
\hline 26 & 0.014 & 0.01 \\
\hline 27 & 0.014 & 0.01 \\
\hline 28 & 0.026 & 0.0186 \\
\hline 29 & 0.026 & 0.0186 \\
\hline 30 & 0 & 0 \\
\hline 31 & 0 & 0 \\
\hline 32 & 0 & 0 \\
\hline 33 & 0.014 & 0.01 \\
\hline 34 & 0.0095 & 0.014 \\
\hline 35 & 0.006 & 0.004 \\
\hline 36 & 0 & 0 \\
\hline 37 & 0.026 & 0.01855 \\
\hline 38 & 0 & 0 \\
\hline 39 & 0.024 & 0.017 \\
\hline 40 & 0.024 & 0.017 \\
\hline 41 & 0.0012 & 0.001 \\
\hline 42 & 0 & 0 \\
\hline 43 & 0.006 & 0.0043 \\
\hline 44 & 0 & 0 \\
\hline 45 & 0.03922 & 0.0263 \\
\hline 46 & 0.03922 & 0.0263 \\
\hline 47 & 0 & 0 \\
\hline
\end{tabular}




\begin{tabular}{|c|c|c|}
\hline Bus Number & Load (MW) & Load (MVAr) \\
\hline 48 & 0.079 & 0.0564 \\
\hline 49 & 0.3847 & 0.2745 \\
\hline 50 & 0.3847 & 0.2745 \\
\hline 51 & 0.0405 & 0.0283 \\
\hline 52 & 0.0036 & 0.0027 \\
\hline 53 & 0.00435 & 0.0035 \\
\hline 54 & 0.0264 & 0.019 \\
\hline 55 & 0.024 & 0.0172 \\
\hline 56 & 0 & 0 \\
\hline 57 & 0 & 0 \\
\hline 58 & 0 & 0 \\
\hline 59 & 0.1 & 0.072 \\
\hline 60 & 0 & 0 \\
\hline 61 & 1.244 & 0.888 \\
\hline 62 & 0.032 & 0.023 \\
\hline 63 & 0 & 0 \\
\hline 64 & 0.227 & 0.162 \\
\hline 65 & 0.059 & 0.042 \\
\hline 66 & 0.018 & 0.013 \\
\hline 67 & 0.018 & 0.013 \\
\hline 68 & 0.028 & 0.02 \\
\hline 69 & 0.028 & 0.02 \\
\hline
\end{tabular}


Table B.3 Branch Data for IEEE 69-bus System

\begin{tabular}{|c|c|c|c|c|}
\hline Branch \# & From & To & Resistance (per unit) & Reactance (per unit) \\
\hline 1 & 1 & 2 & 0.0005 & 0.0012 \\
\hline 2 & 2 & 3 & 0.0005 & 0.0012 \\
\hline 3 & 3 & 4 & 0.0015 & 0.0036 \\
\hline 4 & 4 & 5 & 0.0251 & 0.0294 \\
\hline 5 & 5 & 6 & 0.366 & 0.1864 \\
\hline 6 & 6 & 7 & 0.3811 & 0.1941 \\
\hline 7 & 7 & 8 & 0.0922 & 0.047 \\
\hline 8 & 8 & 9 & 0.0493 & 0.0251 \\
\hline 9 & 9 & 10 & 0.819 & 0.2707 \\
\hline 10 & 10 & 11 & 0.1872 & 0.0619 \\
\hline 11 & 11 & 12 & 0.7114 & 0.235 \\
\hline 12 & 12 & 13 & 1.03 & 0.34 \\
\hline 13 & 13 & 14 & 1.044 & 0.345 \\
\hline 14 & 14 & 15 & 1.058 & 0.3496 \\
\hline 15 & 15 & 16 & 0.1966 & 0.065 \\
\hline 16 & 16 & 17 & 0.3744 & 0.1238 \\
\hline 17 & 17 & 18 & 0.0047 & 0.0016 \\
\hline 18 & 18 & 19 & 0.3276 & 0.1083 \\
\hline 19 & 19 & 20 & 0.2106 & 0.069 \\
\hline
\end{tabular}




\begin{tabular}{|c|c|c|c|c|}
\hline Branch \# & From & To & Resistance (per unit) & Reactance (per unit) \\
\hline 20 & 20 & 21 & 0.3416 & 0.1129 \\
\hline 21 & 21 & 22 & 0.014 & 0.0046 \\
\hline 22 & 22 & 23 & 0.1591 & 0.0526 \\
\hline 23 & 23 & 24 & 0.3463 & 0.1145 \\
\hline 24 & 24 & 25 & 0.7488 & 0.2475 \\
\hline 25 & 25 & 26 & 0.3089 & 0.1021 \\
\hline 26 & 26 & 27 & 0.1732 & 0.0572 \\
\hline 27 & 27 & 28 & 0.0044 & 0.0108 \\
\hline 28 & 28 & 29 & 0.064 & 0.1565 \\
\hline 29 & 29 & 30 & 0.3978 & 0.1315 \\
\hline 30 & 30 & 31 & 0.0702 & 0.0232 \\
\hline 31 & 31 & 32 & 0.351 & 0.116 \\
\hline 32 & 32 & 33 & 0.839 & 0.2816 \\
\hline 33 & 33 & 34 & 1.708 & 0.5646 \\
\hline 34 & 34 & 35 & 1.474 & 0.4873 \\
\hline 35 & 36 & 37 & 0.064 & 0.1565 \\
\hline 36 & 37 & 38 & 0.1053 & 0.123 \\
\hline 37 & 38 & 39 & 0.0304 & 0.0355 \\
\hline 38 & 39 & 40 & 0.0018 & 0.0021 \\
\hline 39 & 40 & 41 & 0.7283 & 0.8509 \\
\hline 40 & 41 & 42 & 0.31 & 0.3623 \\
\hline 41 & 42 & 43 & 0.041 & 0.0478 \\
\hline
\end{tabular}




\begin{tabular}{|c|c|c|c|c|}
\hline Branch \# & From & To & Resistance (per unit) & Reactance (per unit) \\
\hline 42 & 43 & 44 & 0.0092 & 0.0116 \\
\hline 43 & 44 & 45 & 0.1089 & 0.1373 \\
\hline 44 & 45 & 46 & 0.0009 & 0.0012 \\
\hline 45 & 4 & 47 & 0.0034 & 0.0084 \\
\hline 46 & 47 & 48 & 0.0851 & 0.2083 \\
\hline 47 & 48 & 49 & 0.2898 & 0.7091 \\
\hline 48 & 49 & 50 & 0.0822 & 0.2011 \\
\hline 49 & 8 & 51 & 0.0928 & 0.0473 \\
\hline 50 & 51 & 52 & 0.3319 & 0.1114 \\
\hline 51 & 9 & 53 & 0.174 & 0.0886 \\
\hline 52 & 53 & 54 & 0.203 & 0.1034 \\
\hline 53 & 54 & 55 & 0.2842 & 0.1447 \\
\hline 54 & 55 & 56 & 0.2813 & 0.1433 \\
\hline 55 & 56 & 57 & 1.59 & 0.5337 \\
\hline 56 & 57 & 58 & 0.7837 & 0.263 \\
\hline 57 & 58 & 59 & 0.3042 & 0.1006 \\
\hline 58 & 59 & 60 & 0.3861 & 0.1172 \\
\hline 59 & 60 & 61 & 0.5075 & 0.2585 \\
\hline 60 & 61 & 62 & 0.0974 & 0.0496 \\
\hline 61 & 62 & 63 & 0.145 & 0.0738 \\
\hline 62 & 63 & 64 & 0.7105 & 0.3619 \\
\hline 63 & 64 & 65 & 1.041 & 0.5302 \\
\hline
\end{tabular}




\begin{tabular}{c|c|c|c|c}
\hline Branch \# & From & To & Resistance (per unit) & Reactance (per unit) \\
\hline 64 & 11 & 66 & 0.2012 & 0.0611 \\
\hline 65 & 66 & 67 & 0.0047 & 0.0014 \\
\hline 66 & 12 & 68 & 0.7394 & 0.2444 \\
\hline 67 & 68 & 69 & 0.0047 & 0.0096 \\
\hline 68 & 3 & 36 & 0.0047 & 0.0096 \\
\hline
\end{tabular}




\section{REFERENCE LIST}

[1] W. H. Kersting, "Distribution System Modeling and Analysis", $2^{\text {nd }}$ Ed., CRC Press, 2006

[2] [Online]. Available: http://www.theiet.org/resources/conferences/energy/cired2013-usb.cfm

[3] J. S. Jha, S. Sen, Improvement of power distribution system - A few aspects, NPSC, 236-240, 2002.

[4] Toronto Hydro-Electric System Limited, "Distribution system - Asset Management Approach," EB-2011-0144, 2011.

[5] N. Etherden and M. H. J. Bollen, "Dimensioning of energy storage for increased integration of wind power,” IEEE Trans. Sustain. Energy, vol. 4, no. 3, pp. 546-553, 2013.

[6] S. Gill, I. Kockar, and G. W. Ault, "Dynamic optimal power flow for active distribution networks," IEEE Trans. Power Syst., vol. 29, no. 1, pp. 121-131, Jan. 2014.

[7] S. Carr, G. C. Premier, A. J. Guwy, R. M. Dinsdale, and J. Maddy, "Energy storage for active network management on electricity distribution networks with wind power," IET Renew. Power Gener., vol. 8, no. 3, pp. 249-259, 2014.

[8] S. W. Alnaser, and L. F. Ochoa, "Optimal Sizing and Control of Energy Storage in Wind PowerRich Distribution Networks”, IEEE Trans. On power systems, vol 31, no. 3, Apr. 2016

[9] A. Gabash and P. Li, "Active-reactive optimal power flow in distribution networks with embedded generation and battery storage," IEEE Trans. Power Syst., vol. 27, no. 4, pp. 20262035, Nov. 2012.

[10]A. Gabash and P. Li, "Flexible optimal operation of battery storage systems for energy supply networks,” IEEE Trans. Power Syst., vol. 28, no. 3, pp. 2788-2797, Aug. 2013. 
[11] A. Gabash and P. Li, "On the control of main substations between transmission and distribution systems," in Proc. 14th EEEIC, Krakow, Poland, May 10-12, 2014, pp. 280-285.

[12] M. Sadeghi, A. Ahmadian, and M. A. Golkar, "Optimal Storage Planning in Active Distribution Network Considering Uncertainty of Wind Power Distributed Generation” IEEE Trans. Power Syst., vol. 31, no. 1, pp. 304-316, Jan. 2016.

[13]C. Chen, S. Duan, T. Cai, B. Liu, and G. Hu, "Optimal allocation and economic analysis of energy storage system in microgrids," IEEE Trans. Power Electron., vol. 26, no. 10, pp. 27622773, Oct. 2011.

[14]F. A. Chacra, P. Bastard, G. Fleury, and R. Clavreul, "Impact of energy storage costs on economical performance in a distribution substation,” IEEE Trans. Power Syst., vol. 24, no. 2, pp. 684-691, May 2005.

[15] S. Wen, H. Lan, Q. Fu, D. C. Yu, and L. Zhang, "Economic allocation for energy storage system considering wind power distribution," IEEE Trans. Power sys., vol. 3, no. 2, pp. 644-652, Mar. 2015.

[16] M. Korpaas, A. T. Holen, and R. Hildrum, "Operation and sizing of energy storage for wind power plants in a market system," Int. J. Elect. Power Energy Syst., vol. 25, no. 8, pp. 599-606, Oct. 2003.

[17] A. S. A. Awad, T. H. M. EL-Fouly, and M. M. A. Salama, "Optimal ESS Allocation and Load Shedding for Improving Distribution System Reliability”, IEEE Trans. Smart Grid, vol. 5, no. 5, pp. 2339-2349, 2014.

[18]C. Abbey and G. Joos, "A stochastic optimization approach to rating of energy storage systems in wind-diesel isolated grids," IEEE Trans. Power Syst., vol. 24, no. 1, pp. 418-426, Feb. 2009. 
[19] S. X. Chen, H. B. Gooi, and M. Q. Wang, "Sizing of energy storage for microgrids," IEEE Trans. Smart Grid, vol. 3, no. 1, pp. 142-151, Mar. 2012.

[20]A. S. A. Awad, T. H. M. EL-Fouly, and M. M. A. Salama, “Optimal ESS allocation for load management application”, IEEE Trans. Power Syst., vol. 30, no. 1, pp. 327-336, Jan. 2015.

[21]L. Jenderlanik, C. Mensmann, D. Giavarra, 2013, Enhancing the Asset Management Process for New Demands of Decentralized Energy Integration, Proceedings CIRED 2013, Stockholm.

[22] O. Gjerde, G. Kjølle, J. G. Hernes, B. Hestnes, J. A. Foosnaes, 2011, "Indicators to monitor and manage electricity distribution system vulnerability", Proceedings CIRED 2011, Frankfurt.

[23] A. A. Solomon, D. M. Kammen, and D. Callaway, "The role of large-scale energy storage design and dispatch in the power grid", Appl. Energy, vol. 134, issue C, pp. 75-89, 2014.

[24] M. C. W. Kintner-Meyer, P. J. Balducci, V. V. Viswanathan, C. Jin, X. Guo, T. B. Nguyen, and F. K. Tuffner, "Energy storage for power systems applications: A regional assessment for the Northwest Power Pool," Technical report, 2010. [Online]. Available: http://www.pnl.gov/main/publications/external/technical_reports/PNNL-19300.pdf

[25] H. Pandzi c, Y. Wang, T. Qiu, Y. Dvorkin, and D. S. Kirschen, "Near optimal method for siting and sizing of distributed storage in a transmission network," IEEE Trans. Power Syst., vol. 30, no. 5, pp. 2288-2300, Sep. 2015.

[26] D. Gayme and U. Topcu, "Optimal power flow with large-scale storage integration," IEEE Trans. Power Syst., vol. 28, no. 2, pp. 709-717, May 2013.

[27] Y. V. Makarov, P. Du, M. C. W. Kintner-Meyer, C. Jin, and H. F. Illian, "Sizing energy storage to accommodate high penetration of variable energy resources," IEEE Trans. Sustain. Energy, vol. 3, no. 1, pp. 34-40, Jan. 2012. 
[28] B. M. Grainger, G. Reed, A. R. Sparacino, and P. T. Lewis, "Power electronics for grid-scale energy storage,” Proc. IEEE, vol. 102, no. 6, pp. 1000-1013, Jun. 2014.

[29] L. S. Vargas, G. Bustos-Turu, and F. Larrain, "Wind power curtailment and energy storage in transmission congestion management considering power plants ramp rates," IEEE Trans. Power Syst., vol. 30, no. 5, pp. 2498-2506, Sep. 2015

[30] K. Baker, G. Hug and X. Li, "Energy storage sizing taking into account forecast uncertainties and receding horizon operation", IEEE Trans. Sustain. Energy, vol. 8, no. 1, pp. 331-340, Jan. 2017.

[31] A. Kargarian, G. Hug and J. Mohammadi, “A multi-time scale co-optimization method for sizing of energy storage and fast-ramping generation”, IEEE Trans. Sustain. Energy, vol. 7, no. 4, pp. 1351-1361, Mar. 2016.

[32] Q. Li, R. Ayyanar and V. Vittal,“Convex optimization for DES planning and operation in radial distribution systems with high penetration of photovoltaic resources", IEEE Trans. Sustain. Energy, vol. 7, no. 3, pp. 985-995, Jan. 2016.

[33] E. Hajipour, M. Bozorg, and M. Fotuhi-Firuzabad. "Stochastic capacity expansion planning of remote microgrids with wind farms and energy storage," IEEE Transactions on Sustainable Energy, no.6, vol.2, pp: 1-8,2015.

[34] Qian Dai, Xiaohui Qin, Jingqi Yang, Pingliang Zeng, Qinyong Zhou, Feng Zhao, "Capacity planning of battery energy storage system within wind farm", Power and Energy Engineering Conference (APPEEC) 2016 IEEE PES Asia-Pacific, pp. 1827-1831, 2016.

[35] Y. Dvorkin, R. Fernández-Blanco, D. S. Kirschen and H. Pandžić "Ensuring Profitability of Energy Storage” IEEE Trans. Power Syst., vol. 32, no. 1, pp. 611 - 623, May 2016. 
[36] J. L. Blackburn, "Protective Relaying: Principles \& Applications", $2^{\text {nd }}$ Ed., Monticello, NY, Marcel Dekker, Inc., 1998

[37]J. D. Glover, "Electric Power Distribution", Encyclopedia of Energy Technology and the Environment, John Wiley \& Sons, New York, NY, 1995

[38] A. J. Pansini, “Electrical Distribution Engineering", $2^{\text {nd }}$ Ed., the Fairmount Press, 1992

[39]G. Narzisi, Evolutionary algorithms: A short Introduction., New York: Courant Institute of Mathematical Sciences New York University, 2008, pp.15-17.

[40]R. Šafarič, (2006). Intelligent Control Techniques in Mechatronics - Genetic algorithms [Online].Available:

http://www.ro.feri.unimb.si/predmeti/int reg/Predavanja/Eng/3.Genetic\%20algorithm/ 18.ht

$\underline{\mathrm{ml}}$

[41]M. Farsadi, T. Sattarpour and A. Yazdani Nejadi, "Optimal placement and operation of BESS in a distribution network considering the net present value of energy losses cost", Electrical and Electronics Engineering (ELECO) 2015 9th International Conference, pp. 434-439, 2015.

[42] Q. Sun, B. Huang, D. Li, D. Ma and Y. Zhang, "Optimal Placement of Energy Storage Devices in Microgrids via Structure Preserving Energy Function", IEEE Transactions on Industrial Informatics, vol. 12, no. 3, pp. 1166-1179, Apr. 2016,

[43] A. Narimani, G. Nourbakhsh, G. F. Ledwich, G. R. Walker, "Storage optimum placement in distribution system including renewable energy resources", Power Engineering Conference (AUPEC) 2016 Australasian Universities, pp. 1-5, 2016.

[44] J. Zhao, Z. Zhu, Y. Yu, C. Lin and W. Wei, "Optimal charging/discharging scheme of battery storage systems in active distribution network", Power and Energy Society General Meeting (PESGM) 2016, pp. 1-5, 2016, ISSN 1944-9933. 
[45] V. Kalkhambkar, R. Kumar and R. Bhakar, "Joint optimal allocation of battery storage and hybrid renewable distributed generation", Power Systems (ICPS) 2016 IEEE 6th International Conference on, pp. 1-6, 2016.

[46] K. Prasad, N.C. Sahoo, R. Ranjan, A. Chaturvedi, A new computational approach for optimal reconfiguration of radial distribution systems using a fuzzy controlled genetic algorithm, Computation and Mathematics in Electrical and Electronic Engineering, 25 (4): 862 - 882, 2006.

[47] R. Srinivasa Rao, S. V. L. Narasimham, M. Ramalinga Raju and A. Srinivasa Rao, Optimal network reconfiguration of large-scale distribution system using harmony search algorithm, IEEE Transactions on Power System, 26 (3): 1080 -1088, 2011.

[48] R. T. F. A. King, B. Radha and H. C. S. Rughooputh, A fuzzy logic controlled genetic algorithm for optimal electrical distribution network reconfiguration, IEEE International Conference on Networking, Sensing and Control, 1: 577 - 582, Mar. 2004.

[49] E. M. Carreno, R. Romero, A. Padilha-Feltrin, An Efficient Codification to Solve Distribution Network Reconfiguration for Loss Reduction Problem, IEEE Transactions on Power Systems, 23 (4): 1542 - 1551, Nov. 2008.

[50] A. Mendes, N. Boland, P. Guiney and C. Riveros, Switch and Tap-Changer Reconfiguration of Distribution Networks Using Evolutionary Algorithms, IEEE Transactions in Power Systems, 28 (1): 85 - 92, 2013.

[51] A. C. Santos, A. C. B. Delbem, J. B. A. London, N. G. Bretas, Node-Depth Encoding and Multiobjective Evolutionary Algorithm Applied to Large-Scale Distribution System Reconfiguration, IEEE Transactions on Power Systems, 25 (3): 1254 - 1265, Aug. 2010. 
[52] C. Cheng-Chieh and T. Men-Shen, Application of Novel Charged System Search With Real Number String for Distribution System Loss Minimization, IEEE Transactions on Power Systems, 28 (4): 3600 - 3609, Nov. 2013.

[53] T. Inoue, K. Takano, T. Watanabe, J. Kawahara, R. Yoshinaka, A. Kishimoto, K. Tsuda, S. I. Minato and Y. Hayashi, Distribution Loss Minimization With Guaranteed Error Bound, IEEE Transactions on Smart Grid, 5 (1): 102 - 111, Jan. 2014.

[54] C. Wang and Y. Gao, Determination of Power Distribution Network Configuration Using NonRevisiting Genetic Algorithm, IEEE Transactions on Power Systems, 28 (4): 3638 - 3648, Nov. 2013.

[55] H. D. de Macêdo Braz and B. Alencar de Souza, Distribution network reconfiguration using genetic algorithms with sequential encoding: Subtractive and additive approaches, IEEE Transactions in Power Systems, 26(2): 582-593, 2011.

[56] K. Nara, A. Shioss, M. Kitagwa and T. Ishihwara, Implementation of GA for distribution system loss minimum reconfiguration, IEEE Transaction on Power Systems, 7(3): $1044-1051$. 1992.

[57] W. Wu-Chang, T. Men-Shen, Application of Enhanced Integer Coded Particle Swarm Optimization for Distribution System Feeder Reconfiguration, IEEE Transactions on Power Systems, 26(3): $1591-1599.2011$.

[58] R. Rajan and B. Venkatesh, Optimal Radial Distribution System Reconfiguration using Fuzzy adaptation of Evolutionary Programming, Electrical Power and Energy Systems, 25: 775-780, 2003.

[59] T.Q.D. Khoa, B.T.T. Phan, Ant colony search-based loss minimum for reconfiguration of distribution systems, Power India Conference, 1:6, 2006. 
[60] B. Enacheanu, B. Raison, C. Raphael, O. Devaux, W. Bienia, N. HadjSaid, Radial Network Reconfiguration Using Genetic Algorithm Based on the Matroid Theory, Power Systems, IEEE Transactions, 23(1): 186 - 195, Feb. 2008.

[61] J. S. Savier and D. Das, Impact of network reconfiguration on loss allocation of radial distribution systems, IEEE Transactions on Power Delivery, 22(4): 2473-2480, 2007.

[62] G. Narzisi, Evolutionary algorithms: A short Introduction., New York: Courant Institute of Mathematical Sciences New York University, 15-17, 2008.

[63] J. F. Franco, M. J. Rider, M. Lavorato and R. Romero, A Mixed-Integer LP Model for the Reconfiguration of Radial Electric Distribution Systems Considering Distributed Generation, Electric Power Systems Research, 97, 51 - 60, Apr. 2013.

[64] Electrical Power Distribution Reliability Richard E. Brown page 229

[65] Literature Review and Analysis of Electric Distribution Overhead to Underground Conversion, (2007, Feb. 28) [Online]. Available: http:/grouper.ieee.org/groups/td/dist/sd/doc/2007-02-Undergrounding-Assessment.pdf

[66]DOE/EPRI 2013 Electricity Storage Handbook in Collaboration with NRECA (2013, Jul.) [Online] Available: http://www.sandia.gov/ess/publications/SAND2013-5131.pdf

[67]B. Venkatesh, R. Ranjan, and H. B. Gooi, "Optimal reconfiguration of radial distribution systems to maximize loadability," IEEE Transactions on Power Systems, vol. 19, no. 1, pp. 260266, Feb. 2004.

[68] S. Leyffer, G. López-Calva and J. Nocedal, Interior Methods for Mathematical Programs with Complementarity Constraints, SIAM Journal on Optimization, 17(1): 52-77, 2006.

[69] S. Leyffer, Complementarity constraints as nonlinear equations: Theory and numerical experience, Optimization with Multivalued Mappings, 2: 169-208, 2006. 
[70] J. Z. Zhu, Optimal reconfiguration of electrical distribution network using the refined genetic algorithm, Electric Power Systems Research, 62: 37-42, 2002.

[71]D. Zhang, Z. Fu, and L. Zhang, An improved TS algorithm for loss minimum reconfiguration in large-scale distribution systems, Electric Power Systems Research, 77: 685-694, 2007.

[72]M. Andervazh, J. Olamaei, M. Haghifam, Adaptive multi-objective distribution network reconfiguration using multi-objective discrete particles swarm optimisation algorithm and graph theory, IET Generation, Transmission \& Distribution, 7(12): 1367-1382, Dec. 2013

[73]Modern Optimization Techniques with Applications in Electric Power Systems, Abdel-Aal Hassan Mantawy and Soliman Abdel-hady Soliman, 2011

[74]The Coming Electricity Storage Revolution. Available: http://www.agmrc.org/renewableenergy/renewable-energy/the-coming-electricity-storage-revolution/ 